\title{
Rock Climbers in the New River Gorge: Attitudes toward Management Actions and Trust of Park Managers
}

\author{
Kathryn M. McKenney \\ West Virginia University
}

Follow this and additional works at: https://researchrepository.wvu.edu/etd

\section{Recommended Citation}

McKenney, Kathryn M., "Rock Climbers in the New River Gorge: Attitudes toward Management Actions and Trust of Park Managers" (2013). Graduate Theses, Dissertations, and Problem Reports. 623.

https://researchrepository.wvu.edu/etd/623

This Thesis is protected by copyright and/or related rights. It has been brought to you by the The Research Repository @ WVU with permission from the rights-holder(s). You are free to use this Thesis in any way that is permitted by the copyright and related rights legislation that applies to your use. For other uses you must obtain permission from the rights-holder(s) directly, unless additional rights are indicated by a Creative Commons license in the record and/ or on the work itself. This Thesis has been accepted for inclusion in WVU Graduate Theses, Dissertations, and Problem Reports collection by an authorized administrator of The Research Repository @ WVU. For more information, please contact researchrepository@mail.wvu.edu. 
Rock Climbers in the New River Gorge: Attitudes toward Management Actions and Trust of Park Managers

\author{
Kathryn M. McKenney
}

Thesis submitted to the Davis College of Agriculture, Natural Resources, and Design at West Virginia University in partial fulfillment of the requirements for the degree of

Master of Science

in

Forestry Recreation, Parks and Tourism Resources

Thesis Committee:

Dr. David Smaldone, Chair

Dr. Steven Selin

Dr. Robert Burns

Division of Forestry and Natural Resources

Morgantown, West Virginia 2013

Keywords: Rock climbing, segmentation, specialization, social sub-worlds, trust, management actions

Copyright 2013 Kathryn McKenney 


\author{
ABSTRACT \\ Rock Climbers in the New River Gorge: Attitudes toward Management Actions \\ And Trust of Park Managers \\ Kathryn M. McKenney
}

The New River Gorge National River (NERI), managed by the National Park Service, is a popular destination for whitewater rafters, day hikers, and rock climbers. Located in central West Virginia, about 60 miles east of Charleston, NERI contains over 1700 climbing routes. This thesis is part of a larger study, funded by the National Park Service, which studied geologic, botanical, and recreational components to cliff resources in NERI. This thesis compared the low impact behaviors and management attitudes of hikers and rock climbers, and further segments rock climbers by climbing style, experience level, learning environment, and learning style. This thesis also compared the levels of trust that hikers and climbers have in various sources for information about cliff resource management. Visitors were surveyed at four trailheads in NERI and responded to a variety of statements evaluating their attitudes toward low impact behaviors and cliff resource management, as well as their trust of different sources when it came to obtaining reliable information on cliff resource management. Climbers in general, compared to hikers, were found to have less trust in managers, more negative attitudes toward management actions, and more knowledge of low impact behaviors. Traditional climbers in particular were the least trusting of managers, but had the greatest knowledge of low impact behaviors. 


\section{$\underline{\text { Acknowledgements }}$}

I would first like to thank the John Perez, Robin Snyder, and Shari Quinn of the National Park Service for their support and consultation on this project. Their investment in this project and their answers to my numerous questions were crucial to the writing process.

I would also like to thank my advisor and thesis chair, Dr. David Smaldone, who was instrumental in the completion of this thesis. Over the past two years, he has challenged me and encouraged me to be a better student, teacher, and researcher. His advice and support were invaluable and have made my graduate experience a positive one. I would also like to thank my other committee members, Dr. Steven Selin and Dr. Robert Burns, for their incredible support during the thesis writing process. They were always willing to listen to my ideas and provide useful advice.

Over the past two years, I have benefited from supportive family and friends who supported and encouraged me. I would like to thank my parents and sister, who were always willing to lend an ear when I needed it the most. I would also like to thank my good friend Kelly, and my other good friend Kelly, for providing advice, support, perspective, and occasional proofreading services. You both helped me stay sane, and made the graduate school experience more enjoyable. Lastly, I would like to thank my wonderful boyfriend, Dan, for all of his support over the past two years, as well as his patience and understanding throughout the entire grad school process. 


\section{$\underline{\text { Table of Contents }}$}

Chapter 1: Introduction 1

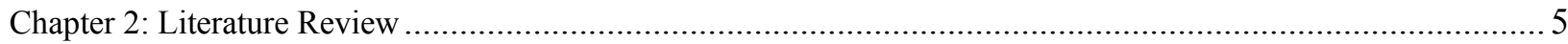

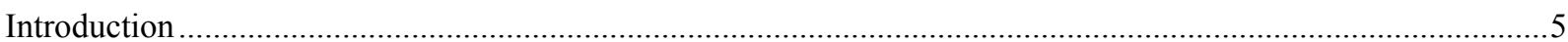

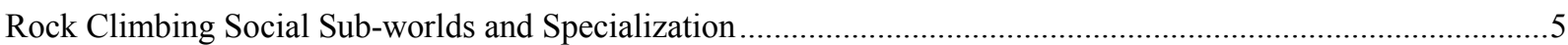

Attitudes toward Resource Impacts and Management Actions ...................................................................6



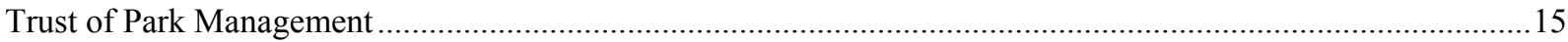

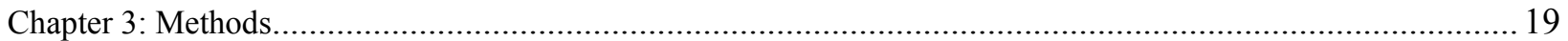

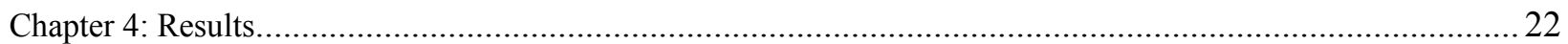

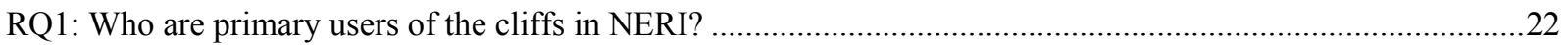

RQ2: Do climbers and hikers differ in their attitudes toward cliff resource management? ..............................25

RQ3: Do different sub-groups (types) of climbers differ in their attitudes toward cliff resource management? ....29

RQ4: Do climbers of different climbing abilities differ in their attitudes toward cliff resource management? ......35

RQ5: Does where (indoors vs. outdoors) climbers learn to climb influence their attitudes toward cliff resource management?

RQ6: Does how (formally trained vs. informally) climbers learn to climb influence their attitudes toward cliff resource management? .......

RQ7: Do hikers and climbers differ in who they trust for information on cliff resources?

RQ1: Who are primary users of the cliffs in NERI?

RQ2: Do climbers and hikers differ in their attitudes toward cliff resource management?

RQ3: Do different sub-groups of climbers differ in their attitudes toward cliff resource management? .

RQ4: Do climbers of different climbing abilities differ in their attitudes toward cliff resource management? ......56

RQ5: Does where (indoors vs. outdoors) climbers learn to climb influence their attitudes toward cliff resource management?

RQ6: Does how (formally trained vs. informally) climbers learn to climb influence their attitudes toward cliff resource management?

RQ7: Do hikers and climbers differ in who they trust for information on cliff resources?

Conclusions.

References

Appendix A: Hiking Survey Instrument

Appendix B: Climbing Survey Instrument 


\section{Tables}

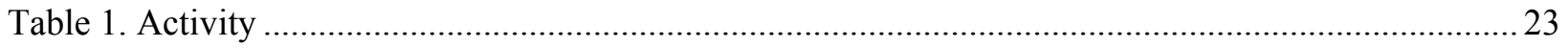

Table 2. Age

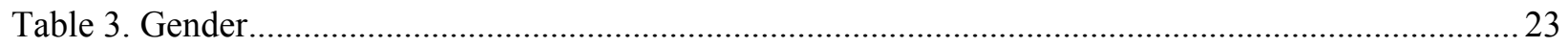

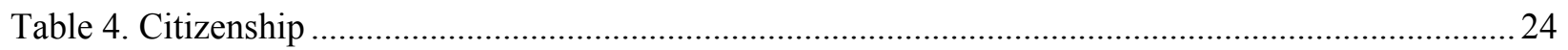

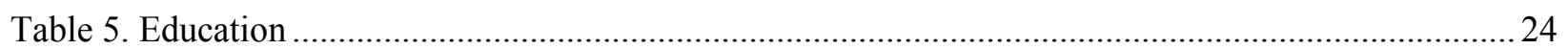

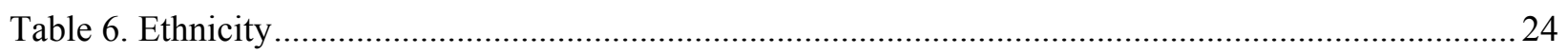

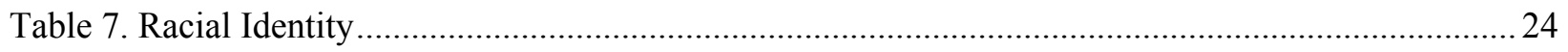

Table 8. Low Impact Behavior Attitudes by Type of Activity …...........................................................26

Table 9. Management Attitudes by Type of Activity ............................................................................28

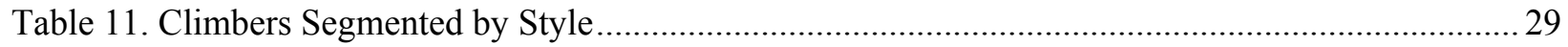

Table 12. Low Impact Behavior Attitudes by Type of Climber ........................................................... 30

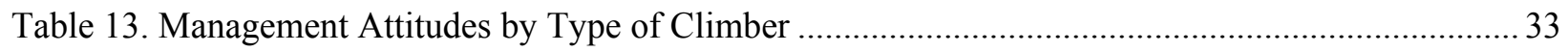

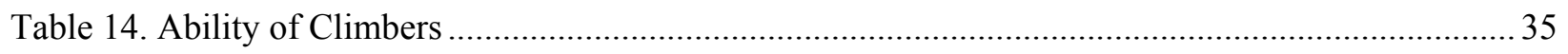

Table 15. Low Impact Behavior Attitudes by Climbing Ability .......................................................... 36



Table 17. Climbers Segmented by Learning Environment .................................................................... 40

Table 18. Low Impact Behavior Attitudes by Learning Environment..................................................... 41

Table 19. Management Attitudes by Learning Environment................................................................. 42

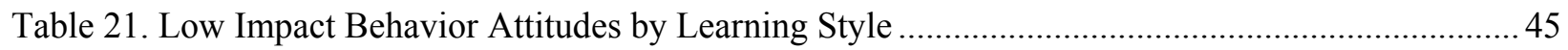

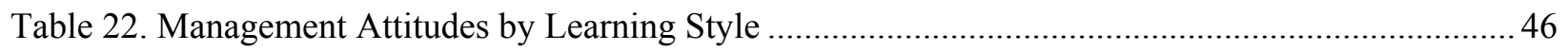

Table 23. Chi-square Analysis of Visitors' Trust of Cliff Management Resources ................................. 48 


\section{Chapter 1: Introduction}

The New River Gorge National River (NERI) is located in central West Virginia, near Beckley, about 60 miles east of Charleston. It has been managed by the National Park Service since 1976, when it was designated a National River. The area managed by the NPS spans 70,000 acres along 53 miles of the New River. Since before the designation of NERI as a National River, the area has been a popular destination for whitewater rafting, kayaking, hiking, and rock climbing. A band of cliffs follows the river on both sides of the gorge, allowing plenty of opportunities for day hiking, sightseeing, and rock climbing. NERI currently contains more than 2400 established climbing routes (Williams, 2010) and a wide variety of hiking trails for users of all abilities. In addition to hosting thousands of recreationists each year, it is also home to 11 rare species of plants and animals, as well as the Virginia big-eared bat and the Indiana bat, both of which are endangered.

As climbing increases in popularity and more people come to NERI for the purpose of rock climbing, various human impacts have become obvious. Increased use of cliff areas has led to the creation of social or unofficial trails, trampling of vegetation, vegetation loss, and erosion. Climbers have been observed scraping fungi, mosses, and other nonvascular plants from the face of the cliffs. While this is a common practice, it does create a visible impact. Trampling at the top of cliffs and the use of trees as an anchor for top ropes is also detrimental to the trees growing on the edges of cliffs. Additionally, increased hiking along the trails on the canyon rim has also lead to the proliferation of social trails, as well as unofficial overlooks being created as hikers seek views of the gorge.

Due to the increased use and the resulting impacts on the cliff resources in NERI, park managers have begun to explore options for reducing the amount of human impact along the 
cliffs. During the summer of 2011, a visitor survey was conducted at four trailheads within NERI. Both hikers and rock climbers were surveyed in regards to their opinions, attitudes, and preferences for cliff use and visitor impacts. Additionally, they were asked about their levels of trust in various information sources and park management in regards to the management of cliff resources.

This thesis was part of a larger study funded by the NPS in order to assess the recreation, geological, and ecological aspects of the cliff resources in NERI. Ultimately, this multi-year, interdisciplinary project will give NERI managers the information that they need to make decisions regarding the preservation and use of cliff resources. This thesis was based on the second stage of the recreation aspect of the study, and included the use of a visitor survey in order to determine visitors' knowledge of cliff resources, perceptions of human impact, attitudes toward management, and levels of trust toward park managers. The following research questions were examined in order to explore the above aspects of the study:

1. Who are primary users of the cliffs in NERI?

2. Do climbers and hikers differ in their attitudes toward cliff resource management?

3. Do different types of climbers differ in their attitudes toward cliff resource management?

4. Do climbers of different climbing abilities differ in their attitudes toward cliff resource management?

5. Does where (indoors vs. outdoors) climbers learn to climb influence their attitudes toward cliff resource management?

6. Does how (formally trained vs. informally) climbers learn to climb influence their attitudes toward cliff resource management? 
7. Do hikers and climbers differ in who they trust for information on cliff resources?

Definition of Terms (Ansari, 2008; Borrie \& Harding, 2002; Monz, 2009; Schuster et al., 2001 ,) Bolting: the act of placing fixed gear.

Bouldering: a style of rock climbing that takes place at low cliffs or boulders and does not use harnesses, fixed gear, or temporary gear. Instead, a thick pad is placed below the climbing site to cushion a climber in the event of a fall.

Boulderer: a rock climber who engages in bouldering exclusively or almost exclusively.

Fixed gear: rock climbing equipment that is permanently attached to the cliff, usually by drilling into the rock and attaching the gear with a bolt. Also referred to as bolts or permanent bolts. Sport climbing: a style of rock climbing that uses gear permanently fixed to the cliff face Sport climbers: rock climbers who engage in sport climbing exclusively or almost exclusively Traditional (trad) climbing: a style of rock climbing in which climbers place temporary gear on the cliff face. The gear is removed by the last climber ascending the cliff. Traditional (trad) climbers: rock climbers who engage in traditional climbing exclusively or almost exclusively.

Hybrid climbing: refers a combination of climbing styles. Many climbers do not engage exclusively in sport climbing, trad climbing, or bouldering, but rather engage in two or three of the above styles with relatively equal frequency.

Hybrid climbers: climbers who engage in sport and trad climbing approximately equally Formal instruction: Climbing instruction given by trained, certified guides in a rock gym or controlled outdoor environment. 
Informal instruction: Climbing instruction given by a friend or family member who is not working in a formal capacity.

Climbers are a diverse group in part due to the numerous climbing styles, learning environments, and learning styles they engage in. Additionally, Bryan (1979) found that recreation specialization exists on a continuum, and that recreationists preferences change as they gain more experience with their chosen activity. Due to the diverse and changing nature of climbers as a group, I chose to segment climbers based on climbing style, experience level, learning environment, and learning style in order to identify differences between sub-worlds of climbers. By understanding climbing sub-worlds, managers can better understand the wants and needs of the climbers using their management area, and can target educational materials and management efforts to address any knowledge or low impact behavior gaps found within the climbing community. 


\section{Chapter 2: Literature Review}

\section{$\underline{\text { Introduction }}$}

Natural resource managers are tasked with ensuring compliance with regulations in order to protect the areas they manage. In order to encourage compliance with these regulations, managers must be trustworthy in the eyes of recreationists, and recreationists must have a positive attitude toward management and the actions that management carries out (Borrie $\&$ Harding, 2002; Monz, 2009; Schuster, Thompson, \& Hammit, 2001). Additionally, managers must be sensitive toward recreationists' attitudes toward resource impacts, and take those attitudes into consideration when deciding which management actions to carry out (Schuster et al., 2001). By understanding the characteristics of the recreationists who use the area and their attitudes and opinions, managers can work to collaborate with dedicated users to manage the area in such a way as to protect the natural resource while still providing for the enjoyment of visitors (Borrie \& Harding, 2001; Payton, Fulton, \& Anderson, 2005). This literature review synthesizes a number of articles that demonstrate the need to further understand climbers' attitudes toward cliff resource management and their trust of park managers (Borrie \& Harding, 2001; Monz, 2009; Schuster et al., 2001). These previous studies also discuss the benefits of segmenting users based on activity, ability, and other qualities to better understand their attitudes and trust levels (Borrie \& Harding, 2001; Monz, 2009; Schuster et al. 2001).

\section{$\underline{\text { Rock Climbing Social Sub-worlds and Specialization }}$}

While the internal dynamics of the rock climbing "world" have not been studied extensively, rock climbers have been shown to be an independent, individualistic group with their own social world (Ansari, 2008; Rapelje, 2004; Thompson, 2007). Segmentation by 
climbing style has long been used to attempt to better understand this world, including the motivations, wants, and needs of climbers (Ansari, 2008; Monz, 2009; Rapelje, 2004; Schuster et al., 2001). The discussion that follows is not intended to provide a theoretical framework for this study, but rather to provide context for the survey, results, and recommendations.

Bryan's (1979) recreation specialization theory tells us that recreation specialization exists on a continuum, from general interest and low participation to specialized interest and high participation. As a recreationist become more specialized within their chosen sport, their preferences for equipment, setting, and experience change (Bryan, 2000).

While rock climbers are specialized compared to other recreationists and have a unique social world, they can be further segmented into social sub-worlds based on climbing style and other preferences (Ansari, 2008). Ansari (2008) examined the motivations of climbers within the traditional and sport climbing sub-worlds in order to help managers better understand these groups and design more effective low impact messages for each group. Ansari (2008) found that traditional and sport climbers do have different motivations, and that managers should consider placing different low impact messages at sites depending on which type of climber used the site the most.

$\underline{\text { Attitudes toward Resource Impacts and Management Actions }}$

Understanding the attitudes of recreationists about their recreation experiences is crucial to improving the management of recreation areas (Manning, 1999; Schuster et al., 2001). By understanding these attitudes, managers can potentially identify desired experiences, define standards, identify minimal and optimal conditions, identify impacts to address, and learn about public opinions in regards to resource conditions and management actions. Once these attitudes are identified and understood, management can then target their actions to address issues that 
negatively affect the attitudes of recreationists using public lands. In addition to understanding attitudes, it is also necessary to take the diverse attitudes of different recreation groups into account, as well as account for variability within recreation groups (Manning, 1999; Stankey, 1972). While early recreation studies (c. 1970) suggested that recreationists were not bothered by impacts enough to report them to park management, Manning (2004) has found that today's visitors may be more sensitive to resource impacts. The more managers know about visitors' attitudes, perceptions, and preferences, the better equipped they will be to provide a quality visitor experience (Manning, 2004). Manning (2004) has also found that managers' perceptions of visitors' preferences are often inaccurate, warranting the need for visitor surveys to determine these preferences.

Schuster et al. (2001) studies of rock climbers' attitudes toward management of climbing and the use of bolts in order to gain a better understanding of the diverse attitudes, wants, and needs of the rock climbers across the country. This study sought to assess the attitudinal dimensions of the American rock climber and identify any attitudinal differences among rock climbing subgroups. The authors point out that the attitudes of rock climbers especially need to be understood, due to the estimated 6.1 million climbers in the United States, as of 2010 (Outdoor Industry Foundation, 2010). With the number of climbers increasing, managers are faced with the challenge of maintaining climbing areas while addressing the increased impacts that stem from heavier use.

Schuster et al. (2001) found that traditional climbers differed significantly from sport and hybrid climbers in regards to bolt placement and use, in that they had more reservations about placing permanent bolts. Despite the reservations of traditional climbers, the mean for this group fell relatively low on a five point Likert scale, indicating that the reservations they had were not 
strong. All climbing groups had reservations about managers' understanding of climbing and the treatment of climbing in the management process. However, traditional climbers perceived a greater need for management than sport climbers. In regards to the placement of bolts, all groups differed significantly from each other. Sport climbers were willing to exercise the least discretion with bolts, while traditional climbers were willing to exercise the most discretion. In other words, sport climbers were more in favor of bolt placement, while traditional climbers had greater reservations about the placement of bolts on the cliff face. Hybrid climbers fell in the middle. While sport climbers were willing to exercise less discretion than hybrid and traditional climbers, they still agreed that discretion should be exercised with the placement of permanent bolts.

Schuster et al. (2001) also found that in regards to climbers' self-perception, traditional climbers thought that climbers in general did not adequately practice land stewardship and had negative attitudes toward management in general. Sport climbers held more positive attitudes of climbers than trad climbers. All groups felt that managers did not adequately understand the activity of climbing, climbers did not adequately understand the management process, climbing was not treated fairly in comparison to other activities, and that climbing was micromanaged. Schuster et al. (2001) concluded that because these climbing subgroups had differing attitudes toward management, they should not be treated the same and their diverse attitudes must be accounted for in the management process. They also noted that managers must be careful not to accommodate one user group at the expense of another.

Another visitor survey by Borrie and Harding (2002) in the Bitterroot Valley, Montana also examined climbers' attitudes toward low impact behaviors of themselves and others. However, the main goal of the study was to examine the impact of different messages displayed 
on information boards on the attitudes of climbers. The authors noted that directly controlling resource impacts can conflict with recreationists' expectations of high levels of personal freedom (Hendee, Stankey, \& Lucas, 1990). Directly controlling visitors' actions includes the use of explicit enforcement of regulations in order to ensure visitor compliance (Manning, 1999). In these cases, the visitor has no opportunity to make decisions regarding their behavior because the decision has been made for them. In contrast, indirectly controlling visitors' actions refers to encouraging preferred behavior through educational efforts, or otherwise setting visitors up to make decisions in regards to their actions (Manning, 1999). If not implemented properly, resource management can interfere with the opportunity for recreationists to make decisions about their behavior during their experience, which can be of crucial importance. Hendee et al. (1990) suggested that managers should choose indirect methods of influencing recreationists' behavior. These methods should be designed to both reduce the impact on the natural resource as well as minimize the effect on recreationists' experiences.

Borrie and Harding (2002) also examined a model of decision making, which outlines the steps that recreationists go through when deciding whether to engage in a low impact behavior or not. Based on a cognitive and social psychology model developed by Tourangeau, Rips, and Rasinski (2000), this four stage model describes the decision making process and notes some of the factors that can intervene at any point and disrupt the process. In order to make a decision regarding low impact behavior, recreationists must comprehend the situation, retrieve information, form a judgment, and finally, choose a behavioral response. If recreationists are illequipped to make a decision, such as lacking information, or if they are disrupted at any point in this model, they will not arrive at the appropriate decision. Additionally, if a recreationist like a climber believes that a certain behavior will not be acceptable by others in his or her group, even 
if it is considered a low impact behavior, they will choose not to engage in that behavior (Borrie \& Harding, 2002).

While the study by Borrie and Harding (2002) provides some insights on how managers can best communicate with sub-groups of climbers, more relevant to this thesis, their study also examined attitudes of climbers based on where they were introduced to climbing (indoors or outdoors), how they were introduced to climbing (formal or informal), their style of climbing (top rope or lead), and their equipment (fixed gear or removable gear). The authors of this study used t-tests to compare climbers' perceptions of their own behavior and the behavior of others based on the four variables listed above.

Their study was limited by severe weather that closed the survey area for a large part of the season, resulting in a smaller sample size and inhibiting the researchers from testing two of the three treatment messages. However, the authors of this study were able to compare the attitudes of climbers by segmenting them in different ways. In comparing climbers based on their mode of introduction to rock climbing and their level of experience with climbing (in particular at the research area), they were able to determine that these two variables had a significant impact on a climbers' attitudes toward low impact behavior. Those climbers who were introduced to climbing indoors were significantly more uncomfortable climbing within sight of Native American artifacts, scoring a mean of 2.8 on a 5 point Likert scale measuring comfort climbing near artifacts, compared to climbers who learned outdoors, who had a mean of 4.8. Climbers who were introduced to climbing indoors were also significantly more uncomfortable with the idea of placing a new bolted route. Those who learned to climb outdoors with bolted anchors already in place were also less likely to express disapproval toward someone using a power drill to place a new bolt. 
The authors of this study concluded that although there was not enough information to make recommendations in regards to communication strategies, this study provided valuable insights into climbers' attitudes toward low impact behaviors based on their prior experiences. Additionally, they noted the need for further research in regards to both visitor communication and understanding attitudes toward low impact behavior (Borrie \& Harding, 2002).

In order to better understand climbers' attitudes toward low impact behaviors, it is necessary to understand how they, as a group, feel about resource impacts caused by recreation. Monz (2009) conducted a study assessing climbers' attitudes toward recreation resource impacts in the Adirondack Park's Giant Mountain Wilderness. Climbers were surveyed as to the types of impacts they found objectionable, and climbers' attitudes were compared based on their climbing experience and style.

The Giant Mountain Wilderness study examined resource impacts that were limited to impacts on the cliff face, such as the placement of permanent bolts. Monz (2009) also addressed issues of crowding and the management of climbing in popular climbing areas. In examining these issues, Monz (2009) segmented climbers based on their experience level and style of climbing in order to determine if these variables have any influence on the perceptions of climbers in regards to resource conditions.

Climbers were surveyed at entry points to climbing areas in the Giant Mountain Wilderness in the Adirondack Park during September and October. The survey asked climbers about their preferred climbing style, attitudes toward environmental impacts, importance of wilderness, and attitudes toward the management of climbing areas, especially related to the placement of fixed anchors and bolts. Before climbers were given the survey, they were first asked to list the types of resource impacts that they found to be the most offensive. The most 
frequently mentioned impacts were: appearance of litter, general erosion around the site, impacts to trees from climbing practices or erosion around the roots, and cigarette butts around the climbing area.

Of the resource impacts assessed using Likert scaled questions, damaged trees, trampled vegetation, and top of cliff impacts were reported as offensive most frequently. While erosion and multiple trails were also reported as offensive by the majority of climbers, Monz (2009) noted that a large percentage of climbers were not offended by bare soil at the climbing site (44\%), erosion (36\%), and multiple trails (31\%). Climbers were most likely to be opposed or neutral in regards to the need for official agency management of climbing areas. The majority of climbers were aware that they were climbing in a Wilderness area, and felt that Wilderness was worthwhile. There were no significant differences in climbers' attitudes based on their experience level. The only significant difference that occurred during a comparison of climbers' based on climbing style was in regards to damage to trees, which sport climbers found more offensive than traditional climbers.

Monz (2009) argued that understanding recreationists' attitudes toward resource impacts can provide managers with insights as to where and how to direct management actions. Additionally, recognizing disparities between what visitors consider impact problems and what managers' priorities are in regards to resource impacts can help managers focus visitor education on specific issues (Monz, 2009). Managers can also gauge the quality of recreation experiences by examining resource and social impact issues, and work to improve visitors' experiences by addressing problems early on. Result from Monz (2009) suggest that climbers are aware of recreation resource impacts, yet had some reservations about official management of the area. This, combined with the results of Schuster et al. (2001) and Borrie and Harding (2002), suggests 
that management strategies need to be developed in collaboration with climbers' groups in order to be successful and well received by climbers using the area. Attarian (1999) and Monz (2009) both pointed out that this leads to a larger discussion in regards to how visitor information, particularly from one user group, could help decide indicators and standards of a recreation area. But as Borrie and Harding (2002) cautioned, managers must be careful not to accommodate one user group at the expense of another.

\section{$\underline{\text { NERI Cliff Users }}$}

NPS managers at NERI have been dealing with the challenges of managing cliff resources and visitor use since the inception of NERI. To this day, they struggle to strike a balance that allows for sufficient visitor enjoyment while still protecting the integrity of the cliff resources. Two previous studies on climbers have been conducted at NERI in the last 15 years. Ramthun (2001) focused on satisfaction with facilities, and also assessed a few normative satisfaction indicators. The second, by Attarian (1999) was conducted to determine the characteristics of NERI climbers, develop a profile of climbers who engage in various responsible behaviors, and identify a means of information dispersal that offered the greatest potential for influencing the behavior of climbers. Climbers were surveyed at three climbing access points (Bridge Area, Fern Creek, and Kaymoor). Every fourth climber entering or exiting the climbing area was contacted, briefly interviewed, and then given a survey to fill out and mail back to the interviewer. The survey addressed demographics and trip characteristics, preferences for rock climbing settings, rock climbing behavior and practices, membership in climbing and environmental organizations, the climbing publications they read, and volunteerism.

Attarian (1999) concluded that on average, climbers who visited NERI during the study period had been climbing for six years, and spent an average of 24 days per year climbing in 
NERI specifically, and 54 days per year climbing in other areas. Climbers were also asked to rate their skill level as beginner, intermediate, or expert. Seventy percent considered themselves to be expert climbers. Most applicable to this thesis, Attarian (1999) found that most climbers agreed with the majority of the Leave No Trace (LNT) behaviors and principles that were included in the survey. They also responded negatively to practices such as chiseling handholds or removing vegetation, and responded neutrally on the issue of bolting. Also applicable to this thesis is the information regarding membership in climbing and environmental organizations. Forty-four percent of respondents noted they were members of these types of organizations. Of this $44 \%, 31 \%$ were members of the Access Fund, and 14\% indicated that they were members of the Nature Conservancy or the Sierra Club. Attarian (1999) also found that climbers in NERI were aware of and engaged in volunteer maintenance of climbing areas. However, while over $98 \%$ of respondents agreed that climbers should share the responsibility of maintaining climbing areas, only $65 \%$ had participated in maintenance projects, with only $22 \%$ participating in a project in NERI.

Overall, while NERI climbers said they were well educated in regards to low impact behaviors and seemed to agree that impacts should be limited, Attarian (1999) found that specific knowledge related to LNT principles was limited. He stressed that climbers are a diverse group, and all may not participate in low impact behaviors across the board. According to NERI managers, climbers are currently ignoring regulations, such as removing vegetation, chiseling handholds and creating unofficial trails. In his implications, Attarian (1999) noted that education is the favored approach for making visitors aware of climbing regulations and preferred behavior, as it makes expectations clear while remaining non-authoritarian and still leaves room for climbers to make individual choices. Attarian (1999) was also optimistic about the 
opportunity for collaboration between climbers and managers in NERI, given the high percentage of climbers that agreed that they should share responsibility for climbing area maintenance. Collaboration can provide opportunities to improve communication and create a positive working relationship between climbers and managers (Flora, 2000; Frentz, Voth, Burns, $\&$ Sperry, 2000). Attarian (1999) noted the need for continued research in order to identify factors that contribute to responsible behavior, the role of climbing organizations in promoting responsible practices, and the influence of climbing publications and other sources on responsible behavior.

\section{Trust of Park Management}

One final element related to management of natural resources is trust of managers--if visitors do not trust managers, studies have found they are less likely follow policies and abide by regulations (Payton, Fulton, \& Anderson, 2005; Winter \& Cvetkovich, 2010). While there is some literature examining trust and natural resource management, nothing has been written about the levels of trust that climbers have toward managers. Some studies have been conducted on recreationists in general to gauge their levels of trust in managers, and questions from these surveys were adapted for use in the survey instrument employed by this thesis (Payton et al., 2005; Wynveen, Kyle, \& Theodori 2008).

Winter \& Cvetkovich (2010) reviewed four studies related to trust and natural resource management actions addressing questions that are applicable to the challenges faced by NERI managers. The four studies reviewed were referred to as the Coleville study, the 4 Forest Study, the Community Study, and the Southwest study throughout their paper. All of the studies reviewed found that respondents tended to trust the Forest Service in regards to threatened and endangered species management. In the Coleville study, done in the Coleville watershed by 
Cvetkovich \& Winter (1998), the authors found that visitors with higher levels of trust in managers rate informational posters higher on scales of likelihood of stopping to read the poster, personal relevance, and meaningfulness of information. However, they did not find a relationship between the likelihood of behavior change based on the information presented in the signs and trust levels. Perhaps the most applicable to this thesis is the 4 Forest study. The 4 Forest study was conducted by Winter \& Knap (2001) in four southern California National Forests. The authors employed an onsite visitor survey in the Angeles, Cleveland, Los Padres, and San Bernardino National Forests. The survey asked visitors about preferences for managing recreation for the protection of threatened and endangered species, including their perceptions of the effectiveness of educational messages providing behavioral instructions. The 4 Forest study, unlike the Coleville study, did find an association between perceived effectiveness of messages and trust.

Moving beyond the relationship between trust and communication effectiveness, Winter \& Cvetkovich (2010) examined trust and perceptions of management actions by comparing the 4 Forest study, the Community study, and the Southwest study to each other. Previously, it had been demonstrated that fostering trust could be beneficial for compliance with regulations (Cvetkovich \& Lofstedt, 1999; Siegrist, Cvetkovich, \& Roth, 2000). Managers facing low levels of compliance with regulations can use trust building in order to increase compliance with regulations. Winter \& Cvetkovich (2010) expanded that discussion to include trust as beneficial to facilitating conservation based behaviors. Their findings suggest that levels of trust affect the believability of managers' messages about personal actions that impact the environment. They also found evidence that perceived effectiveness of management actions and approval of those 
actions are impacted by trust. Ultimately, they believe that trust is an essential component of managers' efforts to engage recreationists in protecting public lands.

Also related to trust in natural resource managers, Wynveen et al. (2008) conducted a study examining place bonding and social trust in the Big Thicket National Preserve in Texas in the context of managing feral hog populations. Hunting and trapping feral hogs is a recreational activity that needs to be managed in natural resource areas and managers have begun to work collaboratively with residents to manage the population. Wynveen et al. (2008) studied whether the relationship between place bonding and trust in the BTNP managers was moderated by whether or not the survey respondents trusted the National Park Service in general. This study mailed surveys to a random sample of residents in the counties surrounding BTNP (and unfortunately, their response rate was $14 \%$, potentially limiting the usefulness of their findings). The authors found that the survey respondents did not have a strong place attachment to BTNP, and had a moderate level of trust in the NPS to manage feral hogs. Ultimately, they did not confirm their hypothesis, but they did find evidence that there was a relationship between respondent's place bonding and their trust in the BTNP NPS staff, which differs from previous research by Payton et al. (2005).

Payton et al. (2005) conducted a study in the Sherburne National Wildlife Refuge examining the mediating influence of place attachment and trust on civic action. State agencies have found that collaborative approaches to management have encouraged joint stewardship of public lands, and Payton et al. (2005) hoped to demonstrate that increasing place attachment will indirectly increase civic action by raising trust levels (Frentz et al., 2000). Payton et al. (2005) used the results of a mail back survey to develop a partial mediation model in which both place attachment and trust influenced civic action. Payton et al.'s (2005) findings reinforced what 
previous studies have found, demonstrating a relationship between trust and civic involvement (Anheier \& Kendell, 2002; Brehm \& Rahn, 1997; Flora, 2000).

In summary, previous research has demonstrated climbers are a unique type of recreationist, and that even within the climbing community, there are potentially important differences. User segmentation can be effective in determining attitudinal differences and management preferences among different subgroups of an overall user group, like climbers (Borrie \& Harding, 2002; Schuster et al., 2001). By examining these differences, we can find better ways to accommodate diverse user groups, address specific misconceptions, and target educational efforts to users where they will be most effective. Additionally, previous research has found that trust can be a mediating factor in user involvement and civic action (Payton et al., 2005). Evaluating trust levels can provide valuable insight into user group actions, and fostering trust can improve civic action and compliance with regulations (Payton et al., 2005; Winter \& Cvetkovich, 2010). By examining these variables together, we can make better recommendations to managers in regards to improving educational materials, fostering trust to encourage collaboration with user groups, and collaborating with user groups to improve compliance with regulations in order to help preserve natural resources (Borrie \& Harding, 2002; Payton et al., 2005; Schuster et al., 2001; Winter \& Cvetkovich, 2010). 


\section{Chapter 3: Methods}

This thesis used a visitor survey in order to achieve the purposes stated above. The survey gathered information about recreation activities, importance of facilities, opinions about the value of cliff resources and resource impacts, low impact behaviors, opinions about management preferences, and trust of information sources and resource managers. The questions used for this survey were modified from previous studies in NERI and other recreation areas with similar user impact issues (Attarian, 1999; Monz, 2009; Ramthun, 2002). The two main user groups of the cliffs in NERI are hikers and rock climbers- therefore, two versions of the surveys were created. The majority of questions in the two surveys were similar, although the survey for climbers will contain slight wording modifications and some climbing specific questions. Segmenting users based on their activity made it easier to assess differences between these two groups. Both surveys were reviewed by the WVU Institutional Review Board and NERI staff members prior to implementation, and some changes were made based on their feedback. The climbing survey was then pilot tested on 4 climbers with experience climbing in NERI, final modifications were made, and the survey was finalized for this study.

This survey was used to collect data from NERI users over the age of 18 . The study took place between May and November of 2011. A stratified random sampling approach was used. Stratification occurred by day of week (weekday vs. weekend), time of day (morning vs. evening), and location (trailhead). During the summer season (May through August), surveying took place during the week (Monday through Thursday) and on the weekends (Friday through Sunday), with a total of 4 days sampled over the course of a week ( 7 days). During the fall (September through October), surveying occurred on Saturday and Sunday. Surveying took place between 10:00 am and 5:00 pm or between 1:00 pm and 8:00 pm. These times were 
adjusted slightly in the fall to account for changing daylight hours. Sampling occurred on 69 days between May and October of 2011, although some of these days were interrupted by bad weather. When possible, the interrupted days were made up for on the next fair weather day. Sampling took place at four trailheads in NERI: Fern Creek, Kaymoor, Bubba City, and Bridge Buttress. These locations were selected after discussion with local climbers and NERI staff. These four locations attract climbers and other cliff users (mostly hikers, in this case) as well as a wide range of climbing styles and abilities. One trailhead was surveyed during each sampling day.

The survey measured visitors' attitudes toward the natural resources and resource impacts in NERI, current levels of management, hiking and climbing practices, and levels of trust toward different sources of information about rock climbing, including rangers at NERI. Most of these items were measured on a five-point Likert scale ranging from 1 ('strongly disagree') to 5 ('strongly agree') with a 'Don't Know' option available for all questions. A few of these topics relied on a four-point scale in order to remain consistent with previous studies. In these cases, the scale ranged from 1 ('Not a problem' or 'Strongly distrust') to 4 ('Serious problem' or 'Strongly trust'). The trust section did not include a 'Don't Know' option. Survey questions were adapted from a variety of similar studies, including two studies that took place in NERI (Attarian, 1999; Borrie \& Harding, 2002; Monz, 2009; Ramthun, 2001; Schuster et al., 2001). During each sampling day, a trained surveyor was stationed at the trailhead selected for that day. All visitors leaving the trail during the sampling period were approached using a standard introductory script and asked to fill out a survey. For visitors in groups, the adult with the most recent birthday was asked to complete the survey. Visitors were informed that the survey was voluntary, confidential, and anonymous. If visitor agreed to take the survey, they 
were asked if they were rock climbing that day. Climbers received the climbing survey, while all other users received the hiking survey. In addition to the topics listed above, the survey also included questions about demographics, trip and activity characteristics, awareness and understanding related to cliff resources and impacts, and opinions related to using and managing cliff resources. A contact log was kept to record all visitors that they surveyor spoke with, including those who refused to take the survey. This contact log was used to examine nonresponse bias. Five hundred and fifteen visitors were asked to take the survey, and a total of 325 surveys were collected, resulting in a response rate of $63 \%$. Of those 325 surveys, 315 were usable for this thesis. Data were entered in Microsoft Excel and imported into SPSS for cleaning and analysis.

In order to address the first research question, frequencies were used to paint a picture of the users groups at NERI. To address the second, third, and fourth questions, factor analyses were run in order to identify clusters of pertinent information in regards to attitudes about cliff resource management and trust of managers and/or management actions. User groups were segmented in the following ways: hikers and rock climbers, traditional climbers and sport climbers, climbers who learned formally and climbers who learned informally, and climbers who learned indoors and climbers who learned outdoors. Independent t-tests were used to make comparisons among hikers and rock climbers in regards to their attitudes and opinions toward park management, as well as the level of trust toward managers and other sources of information on cliff management. An analysis of variance was be used to compare different climbing groups to each other on the same issues. 


\section{Chapter 4: Results}

The following chapter presents the results of this thesis. Each research question is discussed, along with the statistics used to analyze each question. Basic demographic information, group characteristics, trip characteristics, and low impact behaviors are reported, as are different groups' opinions on the management of cliff resources in NERI. Finally, the trust levels of hikers and climbers are compared in order to provide an accurate picture of who different user groups trust the most and where they may turn for information regarding cliff resource management and management changes that may affect them.

\section{RQ1: Who are primary users of the cliffs in NERI?}

Survey respondents were asked some socio-demographic questions in order to help better understand the users of NERI. These questions included information about age, gender, race, education, citizenship, and primary activity in NERI. Survey sites were purposefully chose to capture both hikers and climbers, and based on the results, this was accomplished. The results of the analysis show that slightly more than half (51\%) of NERI sample were hikers (see Table 1$)$. Approximately $60 \%$ of all respondents were male, but when broken down by activity, hikers were split evenly by gender while the majority of climbers $(68 \%)$ were male (see Table 2$)$. The majority ( $76 \%$ ) of respondents were age 40 or younger (see Table 3 ). However, hikers had a more diverse age distribution than climbers. Most (86\%) of climbers were age 40 or younger, while only $62 \%$ of hikers were. Survey respondents tended to be well educated, with $70 \%$ having a Bachelor's degree or higher, and 31\% had a master's, doctorate, or professional degree of some 
kind (see Table 4). The majority of respondents were citizens of the United States, with only $4 \%$ reporting that they were citizens of another country (see Table 5). The majority $(91.8 \%)$ of respondents identified themselves as white. Small portions of the sample identified themselves as Asian (4\%), Native American or Alaskan (3\%), Hispanic or Latino (3\%) (see Table 6), Black or African American (0.7\%), and Native Hawaiian or Pacific Islanders (0.7\%) (see Table 7).

Table 1. Activity

\begin{tabular}{l|c|c} 
Activity & Frequency & Valid Percent \\
\hline Climbing & 154 & 48.9 \\
\hline Hiking & 161 & 51.1 \\
\hline Total & 315 & 100 \\
\hline
\end{tabular}

Table 2. Age

\begin{tabular}{|l|c|c|c|c|c|c|}
\hline Age & \multicolumn{2}{c}{ Hikers } & \multicolumn{2}{c}{ Climbers } & \multicolumn{2}{c}{ Total } \\
\hline & Frequency & Valid Percent & Frequency & Valid Percent & Frequency & Valid Percent \\
\hline $18-20$ & 4 & 2.6 & 15 & 10.3 & 19 & 6.3 \\
\hline $21-30$ & 63 & 40.6 & 78 & 53.4 & 141 & 46.8 \\
\hline $31-40$ & 31 & 20.0 & 34 & 23.3 & 65 & 21.6 \\
\hline $41-50$ & 26 & 16.8 & 13 & 8.9 & 39 & 13.0 \\
\hline $51-60$ & 23 & 14.8 & 4 & 2.7 & 27 & 9.0 \\
\hline $61+$ & 8 & 5.2 & 2 & 1.4 & 10 & 3.3 \\
\hline Total & 155 & 100.0 & 146 & 100.0 & 301 & 100.0 \\
\hline
\end{tabular}

Table 3. Gender

Gender

Hikers

Climbers

Total

\begin{tabular}{|l|c|c|c|c|c|c|}
\hline & Frequency & Valid Percent & Frequency & Valid Percent & Frequency & Valid Percent \\
\hline Male & 78 & 50.6 & 102 & 68.0 & 180 & 59.2 \\
\hline Female & 76 & 49.4 & 48 & 32.0 & 124 & 40.8 \\
\hline Total & 154 & 100.0 & 150 & 100.0 & 304 & 100 \\
\hline
\end{tabular}


Table 4. Citizenship

US

Hikers

Climbers

Total

\section{Citizen}

\begin{tabular}{l|c|c|c|c|c|c}
\hline & Frequency & Valid Percent & Frequency & Valid Percent & Frequency & Valid Percent \\
\hline Yes & 151 & 97.4 & 142 & 94.7 & 293 & 96.1 \\
\hline No & 4 & 2.6 & 8 & 5.3 & 12 & 3.9 \\
\hline Total & 155 & 100 & 150 & 100.0 & 305 & 100 \\
\hline
\end{tabular}

Table 5. Education

Education

Hikers

Climbers

Total

\begin{tabular}{l|c|c|c|c|c|c|}
\hline & Frequency & Valid Percent & Frequency & Valid Percent & Frequency & Valid Percent \\
\hline Less than high school & 1 & .6 & 2 & 1.3 & 3 & 1.0 \\
\hline High school graduate & 8 & 5.1 & 10 & 6.7 & 18 & 5.9 \\
\hline $\begin{array}{l}\text { Some college or } \\
\text { associate degree }\end{array}$ & 30 & 19.2 & 40 & 26.7 & 70 & 22.9 \\
\hline $\begin{array}{l}\text { Four year college } \\
\text { degree }\end{array}$ & 56 & 35.9 & 65 & 43.3 & 121 & 39.5 \\
\hline $\begin{array}{l}\text { Masters, doctorate, or } \\
\text { professional degree }\end{array}$ & 61 & 39.1 & 33 & 22.0 & 94 & 30.7 \\
\hline \begin{tabular}{l} 
Total \\
\hline
\end{tabular} & 156 & 100.0 & 150 & 100.0 & 306 & 100.0 \\
\hline
\end{tabular}

Table 6. Ethnicity

$\begin{array}{llll}\text { Are you } & \text { Hikers } & \text { Climbers } & \text { Total } \\ \text { Hispanic } & & \\ \text { or Latino? } & & \end{array}$

\begin{tabular}{l|c|c|c|c|c|c}
\hline & Frequency & Valid Percent & Frequency & Valid Percent & Frequency & Valid Percent \\
\hline Yes & 3 & 1.9 & 5 & 3.4 & 8 & 2.6 \\
\hline No & 152 & 98.1 & 144 & 96.6 & 296 & 97.4 \\
\hline Total & 155 & 100.0 & 149 & 100.0 & 304 & 100 \\
\hline
\end{tabular}

Table 7. Racial Identity

Racial Identity

Hikers

Climbers

Total

\begin{tabular}{l|c|c|c|c|c|c}
\hline & Frequency & Valid Percent & Frequency & Valid Percent & Frequency & Valid Percent* \\
\hline Native American or & 3 & 2 & 5 & 3.5 & 8 & 2.5 \\
\hline
\end{tabular}




\begin{tabular}{l|c|c|c|c|c|c}
\hline Alaskan Native & & & & & & \\
\hline Asian & 3 & 2 & 10 & 7 & 13 & 4.3 \\
\hline $\begin{array}{l}\text { Black or African } \\
\text { American }\end{array}$ & 2 & 1.3 & 0 & 0 & 2 & 0.7 \\
\hline $\begin{array}{l}\text { Native Hawaiian or } \\
\begin{array}{l}\text { Other Pacific } \\
\text { Islander }\end{array}\end{array}$ & 1 & 0.7 & 1 & 0.7 & 2 & 0.7 \\
\hline White & 147 & 96.7 & 135 & 94.4 & 282 & 91.8 \\
\hline
\end{tabular}

*Respondents could choose more than one response, percentages may not add to 100

\section{RQ2: Do climbers and hikers differ in their attitudes toward cliff resource management?}

Independent samples t-tests were conducted in order to determine significant differences in low impact behavior and attitudes towards management between climbers and hikers. A number of statistically significant differences were found (see Tables 8-9). There was a significant difference between hikers $(M=4.6)$ and climbers $(M=4.75)$ in regards to practicing Leave No Trace, with climbers reporting higher levels of agreement with the statement that they "practice Leave No Trace" than hikers. There was also a significant difference between hiker's $(\mathrm{M}=2.55)$ and climber's $(\mathrm{M}=2.27)$ beliefs about the hardiness of soils and plants along the trail.

While both groups overall disagreed that soils and plants were hardy, as a group, climbers agreed more strongly that soils and plants along the trail were hardy and not easily damaged.

Additionally, these two groups significantly differed in their perceptions of trail signage. Hikers $(\mathrm{M}=3.93)$ reported higher levels of agreement than climbers $(\mathrm{M}=3.4)$ with the statement "The park provides clear indications about which trails should not be used". Similarly, there were significant differences between hikers and climbers in regards to the continued use of unofficial trails. Hikers ( $M=4.09)$ reported higher levels of agreement than climbers $(M=3.7)$ with the statement that the "continued use of unofficial trails was a serious threat to plants and soils along the park trails". Finally, there were also significant differences between hikers and 
climbers relating to responsibility towards the park resources. Hikers $(\mathrm{M}=4.59)$ reported higher levels of agreement than climbers $(\mathrm{M}=4.33)$ with the statement "I have a responsibility to help protect the plants and ecosystems in the park".

Table 8. Low Impact Behavior Attitudes by Type of Activity

\begin{tabular}{|c|c|c|c|c|}
\hline & \multicolumn{2}{|c|}{$\begin{array}{c}\text { Mean } \\
(\mathrm{N})\end{array}$} & \multirow[t]{2}{*}{$\mathbf{t}$} & \multirow[t]{2}{*}{$\mathbf{p}$} \\
\hline & Hikers & Climbers & & \\
\hline $\begin{array}{l}\text { I practice Leave No Trace when I } \\
\text { hike/climb. }\end{array}$ & $\begin{array}{l}4.60 \\
(130)\end{array}$ & $\begin{array}{l}4.75 \\
(149)\end{array}$ & -2.011 & $.046^{*}$ \\
\hline $\begin{array}{l}\text { Soils and plants along the park trails are } \\
\text { hardy and not easily damaged. }\end{array}$ & $\begin{array}{l}2.55 \\
(150)\end{array}$ & $\begin{array}{c}2.27 \\
(141)\end{array}$ & 2.039 & $.042 *$ \\
\hline $\begin{array}{l}\text { The park provides clear indications about } \\
\text { which trails should not be used. }\end{array}$ & $\begin{array}{c}3.93 \\
(140)\end{array}$ & $\begin{array}{l}3.40 \\
(142)\end{array}$ & 4.265 & $.000 *$ \\
\hline $\begin{array}{l}\text { I feel a responsibility to help protect the } \\
\text { plants and ecosystems in the park. }\end{array}$ & $\begin{array}{l}4.59 \\
(161)\end{array}$ & $\begin{array}{l}4.33 \\
(148)\end{array}$ & 2.935 & $.004 *$ \\
\hline $\begin{array}{l}\text { The continued use of unofficial visitor } \\
\text { created trails is a serious threat to plants and } \\
\text { soils along the park trails. }\end{array}$ & $\begin{array}{l}4.09 \\
(144)\end{array}$ & $\begin{array}{l}3.70 \\
(140)\end{array}$ & 3.083 & $.002 *$ \\
\hline I always pack out my trash. & $\begin{array}{l}4.91 \\
(160)\end{array}$ & $\begin{array}{l}4.89 \\
(151)\end{array}$ & .466 & .642 \\
\hline I always use official trails. & $\begin{array}{l}4.24 \\
(160)\end{array}$ & $\begin{array}{l}4.36 \\
(150)\end{array}$ & -1.173 & .242 \\
\hline I respect revegetation efforts. & $\begin{array}{l}4.80 \\
(159)\end{array}$ & $\begin{array}{l}4.70 \\
(149)\end{array}$ & 1.845 & .066 \\
\hline I avoid trails that have been closed. & $\begin{array}{l}4.51 \\
(159)\end{array}$ & $\begin{array}{l}4.56 \\
(150)\end{array}$ & -.534 & .594 \\
\hline I try to keep my noise level down. & $\begin{array}{l}4.39 \\
(160)\end{array}$ & $\begin{array}{l}4.41 \\
(151)\end{array}$ & -.245 & .807 \\
\hline I park my vehicle in designated areas. & $\begin{array}{l}4.72 \\
(150)\end{array}$ & $\begin{array}{c}4.71 \\
(159)\end{array}$ & .127 & .899 \\
\hline $\begin{array}{l}\text { I respect seasonal closures at certain crags } \\
\text { for wildlife needs. }\end{array}$ & $\begin{array}{l}4.57 \\
(158)\end{array}$ & $\begin{array}{l}4.42 \\
(147)\end{array}$ & 1.694 & .091 \\
\hline $\begin{array}{l}\text { Compared to other people I saw on this visit, } \\
\text { I am causing less damage to the soils and } \\
\text { vegetation. }\end{array}$ & $\begin{array}{c}3.48 \\
(117)\end{array}$ & $\begin{array}{c}3.53 \\
(135)\end{array}$ & -.424 & .672 \\
\hline $\begin{array}{l}\text { It is ok to walk off the official trails, if I stay } \\
\text { on unofficial trails already created by } \\
\text { visitors. }\end{array}$ & $\begin{array}{l}2.67 \\
(146)\end{array}$ & $\begin{array}{c}2.41 \\
(145)\end{array}$ & 1.900 & .058 \\
\hline
\end{tabular}


${ }^{*} \mathrm{p}<0.050$

Statistically significant differences were also found between climbers and hikers in regards to their opinions on the management of rock climbing and climbing areas (see Table 9). Hikers $(\mathrm{M}=3.94)$ agreed more strongly than climbers $(\mathrm{M}=3.64)$ that there should be "official regulations concerning where, when, and how bolts and fixed anchors should be used". Additionally, hikers ( $\mathrm{M}=4.96)$ agreed more strongly than climbers $(\mathrm{M}=4.36)$ that "all climbing guides services should be certified or accredited". As expected, climbers $(\mathrm{M}=3.75)$ were significantly more familiar with climbing regulations than hikers $(\mathrm{M}=2.62)$.

A number of statements dealt with shared values and goals, as well as trust, related to park managers. Hikers reported statistically significant higher levels of agreement than climbers for all these statements. Hikers $(M=3.68)$ had higher levels of agreement than climbers $(\mathrm{M}=3.19)$ with the statement "NPS managers at NERI share my values". Additionally, hikers $(\mathrm{M}=4.07)$ reported higher levels of agreement than climbers $(\mathrm{M}=3.43)$ with the statement "NPS managers at NERI will do what is right for the park". Further continuing this trend, hikers $(\mathrm{M}=3.73)$ reported higher levels of agreement than climbers $(\mathrm{M}=2.99)$ with the statement "NERI managers have the same goals as me".

Climbers ( $M=3.17)$ reported higher levels of agreement than hikers $(M=2.84)$ with the statement "Climbing is not treated fairly in the management process". Climbers (M=1.98) also reported higher levels of agreement than hikers $(\mathrm{M}=1.67)$ with the statement "Other recreation groups are offended by seeing bolts or anchors" (although both groups disagreed overall). Climbers $(\mathrm{M}=3.06)$ reported lower levels of agreement than hikers $(\mathrm{M}=3.38)$ with the statement "Climbing sizes should be limited in NERI". However, climbers (M=2.84) also reported higher levels of agreement than hikers $(\mathrm{M}=1.97)$ with the statement "Large groups detracted from my 
experience”. Additionally, climbers $(\mathrm{M}=3.47)$ reported lower levels of agreement than hikers $(\mathrm{M}=3.84)$ with the statement "Climbing areas need an official management plan in order to provide sustained use".

Table 9. Management Attitudes by Type of Activity

\begin{tabular}{|c|c|c|c|c|}
\hline & \multicolumn{2}{|c|}{$\begin{array}{c}\text { Mean } \\
\mathbf{N}\end{array}$} & \multirow[t]{2}{*}{$\mathbf{t}$} & \multirow[t]{2}{*}{$\mathbf{p}$} \\
\hline & Hikers & Climbers & & \\
\hline $\begin{array}{l}\text { There should be official regulations } \\
\text { concerning where, when, and how bolts and } \\
\text { fixed anchors should be used. }\end{array}$ & $\begin{array}{c}3.94 \\
(105)\end{array}$ & $\begin{array}{l}3.64 \\
(147)\end{array}$ & 2.426 & $.016^{*}$ \\
\hline $\begin{array}{l}\text { The NPS managers at the NRG share my } \\
\text { values. }\end{array}$ & $\begin{array}{l}3.68 \\
(72)\end{array}$ & $\begin{array}{l}3.16 \\
(97)\end{array}$ & 3.913 & $.000^{*}$ \\
\hline $\begin{array}{l}\text { All climbing guide services should be } \\
\text { required to be certified or accredited. }\end{array}$ & $\begin{array}{l}4.96 \\
(120)\end{array}$ & $\begin{array}{l}4.36 \\
(146)\end{array}$ & 3.107 & $.002^{*}$ \\
\hline $\begin{array}{l}\text { I am familiar with the climbing regulations } \\
\text { at the NRG. }\end{array}$ & $\begin{array}{l}2.62 \\
(93)\end{array}$ & $\begin{array}{l}3.75 \\
(142)\end{array}$ & -7.191 & $.000^{*}$ \\
\hline $\begin{array}{l}\text { Climbing is not treated fairly in the } \\
\text { management process when compared to } \\
\text { other recreation activities at the NRG. }\end{array}$ & $\begin{array}{l}2.84 \\
(48)\end{array}$ & $\begin{array}{l}3.17 \\
(102)\end{array}$ & -2.201 & $.029^{*}$ \\
\hline $\begin{array}{l}\text { Climbing group sizes should be limited at } \\
\text { NRG. }\end{array}$ & $\begin{array}{l}3.38 \\
(94)\end{array}$ & $\begin{array}{l}3.06 \\
(145)\end{array}$ & 2.109 & $.036^{*}$ \\
\hline $\begin{array}{l}\text { Other recreational groups are offended by } \\
\text { seeing climbing bolts or anchors. }\end{array}$ & $\begin{array}{l}1.67 \\
(114)\end{array}$ & $\begin{array}{l}1.98 \\
(111)\end{array}$ & -2.640 & $.009^{*}$ \\
\hline $\begin{array}{l}\text { I trust that the NPS managers at the NRG } \\
\text { will do what is right for the park. }\end{array}$ & $\begin{array}{l}4.07 \\
(118)\end{array}$ & $\begin{array}{l}3.43 \\
(129)\end{array}$ & 5.301 & $.000^{*}$ \\
\hline $\begin{array}{l}\text { Large groups of climbers detracted from my } \\
\text { experience at NRG. }\end{array}$ & $\begin{array}{l}1.97 \\
(114)\end{array}$ & $\begin{array}{l}2.84 \\
(149)\end{array}$ & -6.535 & $.000^{*}$ \\
\hline $\begin{array}{l}\text { Most climbing areas need an official } \\
\text { management plan in order to provide } \\
\text { sustained use. }\end{array}$ & $\begin{array}{c}3.84 \\
(107)\end{array}$ & $\begin{array}{c}3.47 \\
(141)\end{array}$ & 2.740 & $.007^{*}$ \\
\hline $\begin{array}{l}\text { The NPS managers at the NRG have the } \\
\text { same goals as me. }\end{array}$ & $\begin{array}{l}3.73 \\
(67)\end{array}$ & $\begin{array}{l}2.99 \\
(91)\end{array}$ & 4.936 & $.000^{*}$ \\
\hline $\begin{array}{l}\text { Park managers do not have adequate } \\
\text { knowledge of climbing to properly manage it } \\
\text { at the NRG. }\end{array}$ & $\begin{array}{l}2.78 \\
(69)\end{array}$ & $\begin{array}{c}3.20 \\
(100)\end{array}$ & -2.340 & $.021^{*}$ \\
\hline $\begin{array}{l}\text { The impact of bolts and anchors on the } \\
\text { natural resource is minimal. }\end{array}$ & $\begin{array}{l}3.80 \\
(86)\end{array}$ & $\begin{array}{l}4.02 \\
(149)\end{array}$ & -1.720 & .087 \\
\hline The presence of numerous bolts and anchors & 2.11 & 1.91 & 1.694 & .091 \\
\hline
\end{tabular}




\begin{tabular}{|l|c|c|c|c|}
\hline negatively affects my climbing experiences. & $(111)$ & $(150)$ & & \\
\hline Climbers need to be educated concerning & 4.22 & 4.12 & .941 & .347 \\
their role in the management process. & $(118)$ & $(146)$ & & \\
\hline Climber's practice of land stewardship is not & 2.45 & 2.60 & -.993 & .322 \\
\hline adequate. & $(69)$ & $(128)$ & & \\
\hline Poor communication of rules and regulations & 2.76 & 2.99 & -1.546 & .124 \\
\hline is a problem at NRG. & $(66)$ & $(116)$ & & \\
\hline Climbers have a negative attitude toward & 2.41 & 2.70 & -1.591 & .114 \\
\hline management at the NRG. & $(58)$ & $(101)$ & & \\
\hline
\end{tabular}

${ }^{*} \mathrm{p}<0.050$

RQ3: Do different sub-groups (types) of climbers differ in their attitudes toward cliff

resource management?

Climbers were asked about the types of climbing they participated in on the day of the survey, as well as what type of climbing they primarily engage in. Using their response to the question about their predominant type of climbing, climbers were divided into five groups: trad climbers, sport climbers, hybrid climbers, gym climbers, and top ropers (refer to introduction for definitions). A one-way analysis of variance was used to analyze the differences among these five groups in regards to low impact behaviors and climbing management. Post-hoc comparisons using Tukey's test revealed some statistically significant results between various types of climbers.

Table 11. Climbers Segmented by Style

Climbing Style Percent

(N)

\begin{tabular}{l|l} 
& 17.9 \\
Top Roper & $(26)$
\end{tabular}




\begin{tabular}{|c|c|}
\hline Sport & 25.5 \\
& $(37)$ \\
\hline Trad & 17.9 \\
& $(26)$ \\
\hline Gym & 16.6 \\
& $(24)$ \\
\hline Sport and Trad Equally & 19.3 \\
& $(28)$ \\
\hline
\end{tabular}

Significant differences occurred in just six statements overall. Only two significant differences were found related to low impact practices between groups (Table 12). The remaining four statements were related to management (Table 13). All of the statistically significant differences that were found occurred between trad climbers and either gym climbers or top ropers or both (see Tables 12-13), with the exception of the statement "Chiseling holds is an acceptable practice”, where all groups were significantly different from each other. Post-hoc comparisons with Tukey's test revealed that traditional climbers $(M=4.72)$ reported statistically higher levels of agreement than top ropers $(\mathrm{M}=4.08)$ with the statement that they "always use official trails while hiking to climbing sites".

Table 12. Low Impact Behavior Attitudes by Type of Climber
Low Impact Behavior
Mean
F $\quad$ p

(N)

\begin{tabular}{l|c|c|c|c|c|c|c|}
\cline { 1 - 7 } & $\begin{array}{l}\text { Top } \\
\text { roper }\end{array}$ & Sport & Trad & Gym & $\begin{array}{c}\text { Sport } \\
\text { and } \\
\text { trad }\end{array}$ & & \\
\hline $\begin{array}{l}\text { I always use official } \\
\text { trails. }\end{array}$ & $\begin{array}{c}4.08 \mathrm{a} \\
(26)\end{array}$ & $\begin{array}{c}4.44 \mathrm{ab} \\
(36)\end{array}$ & $\begin{array}{c}4.72 \mathrm{~b} \\
(25)\end{array}$ & $\begin{array}{c}4.21 \mathrm{ab} \\
(24)\end{array}$ & $\begin{array}{c}4.50 \mathrm{ab} \\
(28)\end{array}$ & 2.634 & $.037^{*}$ \\
\hline Chiseling holds is an & $1.82 \mathrm{a}$ & $1.31 \mathrm{~b}$ & $1.13 \mathrm{c}$ & $1.86 \mathrm{~d}$ & $1.04 \mathrm{e}$ & 6.775 & $.000^{*}$ \\
\hline acceptable practice. & $(22)$ & $(36)$ & $(24)$ & $(22)$ & $(26)$ & & \\
\hline I always pack out my & 4.81 & 4.92 & 4.92 & 4.79 & 4.96 & .701 & .593 \\
trash. & $(26)$ & $(37)$ & $(25)$ & $(24)$ & $(28)$ & & \\
\hline I respect revegetation & 4.72 & 4.61 & 4.80 & 4.63 & 4.82 & 1.076 & .371 \\
efforts. & $(25)$ & $(36)$ & $(25)$ & $(24)$ & $(28)$ & & \\
\hline I avoid trails that have & 4.32 & 4.59 & 4.80 & 4.46 & 4.64 & 1.791 & .134 \\
\hline
\end{tabular}




\begin{tabular}{|c|c|c|c|c|c|c|c|}
\hline been closed. & $(25)$ & $(37)$ & $(25)$ & (24) & $(28)$ & & \\
\hline $\begin{array}{l}\text { I practice Leave No } \\
\text { Trace when I } \\
\text { hike/climb. }\end{array}$ & $\begin{array}{l}4.60 \\
(25)\end{array}$ & $\begin{array}{l}4.76 \\
(37)\end{array}$ & $\begin{array}{l}4.88 \\
(24)\end{array}$ & $\begin{array}{l}4.75 \\
(24)\end{array}$ & $\begin{array}{l}4.75 \\
(28)\end{array}$ & .838 & .503 \\
\hline $\begin{array}{l}\text { I try to keep my noise } \\
\text { level down. }\end{array}$ & $\begin{array}{l}4.15 \\
(26)\end{array}$ & $\begin{array}{l}4.41 \\
(37)\end{array}$ & $\begin{array}{l}4.44 \\
(25)\end{array}$ & $\begin{array}{l}4.46 \\
(24)\end{array}$ & $\begin{array}{l}4.57 \\
(28)\end{array}$ & 1.158 & .332 \\
\hline $\begin{array}{l}\text { Fixed anchors should be } \\
\text { used to help preserve } \\
\text { the environment. }\end{array}$ & $\begin{array}{l}4.63 \\
(24)\end{array}$ & $\begin{array}{l}4.56 \\
(36)\end{array}$ & $\begin{array}{l}4.64 \\
(25)\end{array}$ & $\begin{array}{l}4.71 \\
(24)\end{array}$ & $\begin{array}{l}4.79 \\
(28)\end{array}$ & .640 & .635 \\
\hline $\begin{array}{l}\text { I comply with local } \\
\text { climbing regulations. }\end{array}$ & $\begin{array}{l}4.44 \\
(25)\end{array}$ & $\begin{array}{l}4.76 \\
(37)\end{array}$ & $\begin{array}{l}4.83 \\
(24)\end{array}$ & $\begin{array}{l}4.75 \\
(24)\end{array}$ & $\begin{array}{l}4.64 \\
(28)\end{array}$ & 1.910 & .112 \\
\hline $\begin{array}{l}\text { Old gear should be } \\
\text { removed from belay and } \\
\text { rappel sites. }\end{array}$ & $\begin{array}{l}4.65 \\
(26)\end{array}$ & $\begin{array}{l}4.56 \\
(36)\end{array}$ & $\begin{array}{l}4.24 \\
(25)\end{array}$ & $\begin{array}{l}4.67 \\
(24)\end{array}$ & $\begin{array}{l}4.46 \\
(28)\end{array}$ & 1.569 & .186 \\
\hline $\begin{array}{l}\text { Permanent anchors } \\
\text { provide easy access to } \\
\text { routes. }\end{array}$ & $\begin{array}{l}4.65 \\
(26)\end{array}$ & $\begin{array}{l}4.49 \\
(37)\end{array}$ & $\begin{array}{l}4.72 \\
(25)\end{array}$ & $\begin{array}{l}4.67 \\
(24)\end{array}$ & $\begin{array}{l}4.29 \\
(28)\end{array}$ & 1.675 & .159 \\
\hline $\begin{array}{l}\text { I park my vehicle in } \\
\text { designated areas. }\end{array}$ & $\begin{array}{l}4.73 \\
(26)\end{array}$ & $\begin{array}{l}4.67 \\
(36)\end{array}$ & $\begin{array}{l}4.84 \\
(25)\end{array}$ & $\begin{array}{l}4.71 \\
(24)\end{array}$ & $\begin{array}{l}4.71 \\
(28)\end{array}$ & .499 & .737 \\
\hline $\begin{array}{l}\text { I always consider the } \\
\text { local area ethics when I } \\
\text { climb. }\end{array}$ & $\begin{array}{l}4.46 \\
(24)\end{array}$ & $\begin{array}{l}4.68 \\
(37)\end{array}$ & $\begin{array}{l}4.84 \\
(25)\end{array}$ & $\begin{array}{l}4.58 \\
(24)\end{array}$ & $\begin{array}{l}4.64 \\
(28)\end{array}$ & 1.526 & .198 \\
\hline $\begin{array}{l}\text { Permanent bolted } \\
\text { anchors should be used } \\
\text { at the top of climbs. }\end{array}$ & $\begin{array}{l}4.42 \\
(26)\end{array}$ & $\begin{array}{l}4.35 \\
(37)\end{array}$ & $\begin{array}{l}4.44 \\
(25)\end{array}$ & $\begin{array}{l}4.79 \\
(24)\end{array}$ & $\begin{array}{l}4.43 \\
(28)\end{array}$ & 1.515 & .201 \\
\hline $\begin{array}{l}\text { It is ok to remove/clean } \\
\text { lichens. }\end{array}$ & $\begin{array}{l}2.45 \\
(22)\end{array}$ & $\begin{array}{l}2.78 \\
(37)\end{array}$ & $\begin{array}{l}3.21 \\
(24)\end{array}$ & $\begin{array}{l}3.13 \\
(23)\end{array}$ & $\begin{array}{l}2.67 \\
(27)\end{array}$ & 1.649 & .166 \\
\hline $\begin{array}{l}\text { It is ok to remove/clean } \\
\text { mosses and other } \\
\text { vegetation. }\end{array}$ & $\begin{array}{l}2.50 \\
(22)\end{array}$ & $\begin{array}{l}2.56 \\
(36)\end{array}$ & $\begin{array}{l}3.12 \\
(25)\end{array}$ & $\begin{array}{l}2.92 \\
(24)\end{array}$ & $\begin{array}{l}2.39 \\
(28)\end{array}$ & 1.710 & .152 \\
\hline $\begin{array}{l}\text { It is ok to secure top } \\
\text { ropes to trees. }\end{array}$ & $\begin{array}{l}3.35 \\
(26)\end{array}$ & $\begin{array}{l}3.05 \\
(37)\end{array}$ & $\begin{array}{l}2.61 \\
(23)\end{array}$ & $\begin{array}{l}2.92 \\
(24)\end{array}$ & $\begin{array}{l}3.18 \\
(28)\end{array}$ & 1.431 & .227 \\
\hline $\begin{array}{l}\text { Permanent anchors } \\
\text { increase the use of } \\
\text { routes too much. }\end{array}$ & $\begin{array}{l}2.45 \\
(22)\end{array}$ & $\begin{array}{l}2.37 \\
(35)\end{array}$ & $\begin{array}{l}2.17 \\
(24)\end{array}$ & $\begin{array}{l}2.50 \\
(24)\end{array}$ & $\begin{array}{l}2.25 \\
(28)\end{array}$ & .462 & .763 \\
\hline $\begin{array}{l}\text { Soils and plants along } \\
\text { the park trails are } \\
\text { hardy and not easily } \\
\text { damaged. }\end{array}$ & $\begin{array}{l}2.48 \\
(23)\end{array}$ & $\begin{array}{l}2.27 \\
(33)\end{array}$ & $\begin{array}{l}2.12 \\
(25)\end{array}$ & $\begin{array}{l}2.38 \\
(21)\end{array}$ & $\begin{array}{l}2.04 \\
(28)\end{array}$ & 1.105 & .357 \\
\hline I respect seasonal & 4.50 & 4.41 & 4.32 & 4.48 & 4.37 & .203 & .937 \\
\hline
\end{tabular}




\begin{tabular}{|c|c|c|c|c|c|c|c|}
\hline $\begin{array}{l}\text { closures at certain crags } \\
\text { for wildlife needs. }\end{array}$ & (24) & (37) & (25) & (23) & (27) & & \\
\hline $\begin{array}{l}\text { The park provides clear } \\
\text { indications about which } \\
\text { trails should not be } \\
\text { used. }\end{array}$ & $\begin{array}{l}3.25 \\
(24)\end{array}$ & $\begin{array}{l}3.43 \\
(35)\end{array}$ & $\begin{array}{l}3.57 \\
(23)\end{array}$ & $\begin{array}{l}3.23 \\
(22)\end{array}$ & $\begin{array}{l}3.50 \\
(28)\end{array}$ & .435 & .783 \\
\hline $\begin{array}{l}\text { Compared to other } \\
\text { people I saw on this } \\
\text { visit, I am causing less } \\
\text { damage to the soils and } \\
\text { vegetation. }\end{array}$ & $\begin{array}{l}3.45 \\
(22)\end{array}$ & $\begin{array}{l}3.61 \\
(33)\end{array}$ & $\begin{array}{l}3.63 \\
(24)\end{array}$ & $\begin{array}{l}3.45 \\
(20)\end{array}$ & $\begin{array}{l}3.50 \\
(26)\end{array}$ & .260 & .903 \\
\hline $\begin{array}{l}\text { Permanent anchors are } \\
\text { not needed. }\end{array}$ & $\begin{array}{c}2.00 \\
24\end{array}$ & $\begin{array}{c}1.75 \\
36\end{array}$ & $\begin{array}{l}1.80 \\
25\end{array}$ & $\begin{array}{c}1.86 \\
22\end{array}$ & $\begin{array}{c}1.68 \\
28\end{array}$ & .485 & .747 \\
\hline $\begin{array}{l}\text { I feel a responsibility to } \\
\text { help protect the plants } \\
\text { and ecosystems in the } \\
\text { park. }\end{array}$ & $\begin{array}{l}4.35 \\
(26)\end{array}$ & $\begin{array}{l}4.11 \\
(35)\end{array}$ & $\begin{array}{l}4.32 \\
(25)\end{array}$ & $\begin{array}{l}4.26 \\
(23)\end{array}$ & $\begin{array}{l}4.57 \\
(28)\end{array}$ & 1.377 & .245 \\
\hline $\begin{array}{l}\text { The continued use of } \\
\text { unofficial visitor created } \\
\text { trails is a serious threat } \\
\text { to plants and soils along } \\
\text { the park trails. }\end{array}$ & $\begin{array}{l}3.67 \\
(24)\end{array}$ & $\begin{array}{l}3.68 \\
(34)\end{array}$ & $\begin{array}{l}3.76 \\
(25)\end{array}$ & $\begin{array}{l}3.62 \\
(21)\end{array}$ & $\begin{array}{l}3.77 \\
(26)\end{array}$ & .082 & .988 \\
\hline $\begin{array}{l}\text { It is ok to walk off the } \\
\text { official trails, if I stay on } \\
\text { unofficial trails already } \\
\text { created by visitors. }\end{array}$ & $\begin{array}{l}2.72 \\
(25)\end{array}$ & $\begin{array}{l}2.08 \\
(36)\end{array}$ & $\begin{array}{l}2.32 \\
(25)\end{array}$ & $\begin{array}{l}2.48 \\
(21)\end{array}$ & $\begin{array}{l}2.61 \\
(28)\end{array}$ & 1.807 & .131 \\
\hline
\end{tabular}

$* \mathrm{p}<0.050$

Within the management questions, 4 statements were significant (see Table 13). Post-hoc comparisons with Tukey's test revealed that trad climbers $(M=4.35)$ were statistically significantly more familiar with climbing regulations than top ropers $(M=3.39)$ and gym climbers $(\mathrm{M}=3.27)$. In addition to being more knowledgeable about climbing regulations, trad climbers were statistically significantly less trustful of managers. Post-hoc comparisons with Tukey's test revealed that different types of climbers displayed statistically significant differences for two statements related to trust of managers and their goals for the park. Trad 
climbers $(\mathrm{M}=3.05)$ rated the statement "I trust that NPS managers at NERI will do what is right for the park" significantly lower than gym climbers $(\mathrm{M}=4.00)$. Additionally, gym climbers $(\mathrm{M}=3.60)$ agreed more strongly than trad climbers $(\mathrm{M}=2.31)$ that NERI managers have the same goals as them. There were also statistically significant differences in regards to the practice of chiseling holds. The sub-groups all differed significantly from each other with respect to the statement "Chiseling holds is an acceptable practice". While all groups disagreed with the practice, top ropers $(\mathrm{M}=1.82)$ and gym climbers (1.86) reported higher levels of agreement than sport climbers $(\mathrm{M}=1.31)$, trad climbers $(\mathrm{M}=1.13)$, and hybrid climbers $(\mathrm{M}=1.04)$.

Table 13. Management Attitudes by Type of Climber

\begin{tabular}{|c|c|c|c|c|c|c|c|}
\hline \multirow[t]{2}{*}{ Management Questions } & \multicolumn{5}{|c|}{$\begin{array}{c}\text { Means } \\
\text { (N) }\end{array}$} & \multirow[t]{2}{*}{$\mathbf{F}$} & \multirow[t]{2}{*}{$\mathbf{p}$} \\
\hline & $\begin{array}{l}\text { Top } \\
\text { roper }\end{array}$ & Sport & Trad & Gym & $\begin{array}{l}\text { Sport } \\
\text { and } \\
\text { trad }\end{array}$ & & \\
\hline $\begin{array}{l}\text { I am familiar with the } \\
\text { climbing regulations at } \\
\text { the NRG. }\end{array}$ & $\begin{array}{c}3.39 \mathrm{a} \\
(23)\end{array}$ & $\begin{array}{c}3.81 \mathrm{abc} \\
(36)\end{array}$ & $\begin{array}{c}4.35 \mathrm{~b} \\
(23)\end{array}$ & $\begin{array}{c}3.27 \mathrm{c} \\
(22)\end{array}$ & $\begin{array}{c}4.00 \\
a b c \\
(27)\end{array}$ & 4.827 & $.001 *$ \\
\hline $\begin{array}{l}\text { Other recreational } \\
\text { groups are offended by } \\
\text { seeing climbing bolts or } \\
\text { anchors. }\end{array}$ & $\begin{array}{c}2.35 \mathrm{a} \\
(17)\end{array}$ & $\begin{array}{c}1.83 \mathrm{ab} \\
(30)\end{array}$ & $\begin{array}{c}1.63 \mathrm{~b} \\
(16)\end{array}$ & $\begin{array}{c}2.40 \mathrm{ab} \\
(20)\end{array}$ & $\begin{array}{c}1.81 \mathrm{ab} \\
(21)\end{array}$ & 2.987 & $.022 *$ \\
\hline $\begin{array}{l}\text { I trust that the NPS } \\
\text { managers at the NRG } \\
\text { will do what is right for } \\
\text { the park. }\end{array}$ & $\begin{array}{c}3.65 \mathrm{ab} \\
(23)\end{array}$ & $\begin{array}{c}3.30 \mathrm{ab} \\
(30)\end{array}$ & $\begin{array}{c}3.05 \mathrm{a} \\
(21)\end{array}$ & $\begin{array}{c}4.00 \mathrm{~b} \\
(20)\end{array}$ & $\begin{array}{c}3.29 \mathrm{ab} \\
(24)\end{array}$ & 2.584 & $.041 *$ \\
\hline $\begin{array}{l}\text { The NPS managers at the } \\
\text { NRG have the same goals } \\
\text { as me. }\end{array}$ & $\begin{array}{c}3.07 \mathrm{ab} \\
(15)\end{array}$ & $\begin{array}{c}2.92 \mathrm{ab} \\
(24)\end{array}$ & $\begin{array}{c}2.31 \mathrm{a} \\
(13)\end{array}$ & $\begin{array}{c}3.60 \mathrm{~b} \\
(15)\end{array}$ & $\begin{array}{c}3.06 \mathrm{ab} \\
(17)\end{array}$ & 3.016 & $.023 *$ \\
\hline $\begin{array}{l}\text { The impact of bolts and } \\
\text { anchors on the natural } \\
\text { resource is minimal. }\end{array}$ & $\begin{array}{l}3.67 \\
(24)\end{array}$ & $\begin{array}{l}4.30 \\
(37)\end{array}$ & $\begin{array}{l}4.12 \\
(25)\end{array}$ & $\begin{array}{l}3.88 \\
(24)\end{array}$ & $\begin{array}{l}4.00 \\
(28)\end{array}$ & 2.106 & .084 \\
\hline $\begin{array}{l}\text { There should be official } \\
\text { regulations concerning } \\
\text { where, when, and how }\end{array}$ & $\begin{array}{l}3.40 \\
(25)\end{array}$ & $\begin{array}{l}3.51 \\
(37)\end{array}$ & $\begin{array}{l}3.54 \\
(24)\end{array}$ & $\begin{array}{l}3.79 \\
(24)\end{array}$ & $\begin{array}{l}3.92 \\
(26)\end{array}$ & 1.070 & .374 \\
\hline
\end{tabular}




\begin{tabular}{|c|c|c|c|c|c|c|c|}
\hline $\begin{array}{l}\text { bolts and fixed anchors } \\
\text { should be used. }\end{array}$ & & & & & & & \\
\hline $\begin{array}{l}\text { The presence of } \\
\text { numerous bolts and } \\
\text { anchors negatively } \\
\text { affects my climbing } \\
\text { experiences. }\end{array}$ & $\begin{array}{l}1.92 \\
(25)\end{array}$ & $\begin{array}{l}1.68 \\
(37)\end{array}$ & $\begin{array}{l}2.12 \\
(25)\end{array}$ & $\begin{array}{l}1.88 \\
(24)\end{array}$ & $\begin{array}{l}2.25 \\
(28)\end{array}$ & 1.810 & .131 \\
\hline $\begin{array}{l}\text { The NPS managers at the } \\
\text { NRG share my values. }\end{array}$ & $\begin{array}{l}3.06 \\
(16)\end{array}$ & $\begin{array}{l}3.23 \\
(26)\end{array}$ & $\begin{array}{l}2.81 \\
(16)\end{array}$ & $\begin{array}{l}3.56 \\
(16)\end{array}$ & $\begin{array}{l}3.19 \\
(16)\end{array}$ & 1.333 & .264 \\
\hline $\begin{array}{l}\text { All climbing guide } \\
\text { services should be } \\
\text { required to be certified } \\
\text { or accredited. }\end{array}$ & $\begin{array}{l}4.08 \\
(24)\end{array}$ & $\begin{array}{l}4.17 \\
(36)\end{array}$ & $\begin{array}{l}4.04 \\
(25)\end{array}$ & $\begin{array}{l}4.27 \\
(22)\end{array}$ & $\begin{array}{l}4.57 \\
(28)\end{array}$ & 1.245 & .295 \\
\hline $\begin{array}{l}\text { Climbing is not treated } \\
\text { fairly in the management } \\
\text { process when compared } \\
\text { to other recreation } \\
\text { activities at the NRG. }\end{array}$ & $\begin{array}{l}3.20 \\
(15)\end{array}$ & $\begin{array}{l}3.04 \\
(25)\end{array}$ & $\begin{array}{l}3.35 \\
(17)\end{array}$ & $\begin{array}{l}2.88 \\
(16)\end{array}$ & $\begin{array}{l}3.05 \\
(21)\end{array}$ & .643 & .633 \\
\hline $\begin{array}{l}\text { Climbing group sizes } \\
\text { should be limited at } \\
\text { NRG. }\end{array}$ & $\begin{array}{l}3.39 \\
(23)\end{array}$ & $\begin{array}{l}2.67 \\
(36)\end{array}$ & $\begin{array}{l}3.29 \\
(24)\end{array}$ & $\begin{array}{l}2.67 \\
(24)\end{array}$ & $\begin{array}{l}3.30 \\
(27)\end{array}$ & 2.296 & .063 \\
\hline $\begin{array}{l}\text { Climbers need to be } \\
\text { educated concerning } \\
\text { their role in the } \\
\text { management process. }\end{array}$ & $\begin{array}{l}4.00 \\
(24)\end{array}$ & $\begin{array}{l}4.11 \\
(35)\end{array}$ & $\begin{array}{l}4.00 \\
(24)\end{array}$ & $\begin{array}{l}4.13 \\
(24)\end{array}$ & $\begin{array}{l}4.29 \\
(28)\end{array}$ & .606 & .659 \\
\hline $\begin{array}{l}\text { Large groups of climbers } \\
\text { detracted from my } \\
\text { experience at NRG. }\end{array}$ & $\begin{array}{l}2.58 \\
(24)\end{array}$ & $\begin{array}{l}2.95 \\
(37)\end{array}$ & $\begin{array}{l}3.12 \\
(25)\end{array}$ & $\begin{array}{l}2.83 \\
(24)\end{array}$ & $\begin{array}{l}2.89 \\
(28)\end{array}$ & .665 & .617 \\
\hline $\begin{array}{l}\text { Most climbing areas need } \\
\text { an official management } \\
\text { plan in order to provide } \\
\text { sustained use. }\end{array}$ & $\begin{array}{l}3.43 \\
(23)\end{array}$ & $\begin{array}{l}3.28 \\
(36)\end{array}$ & $\begin{array}{l}3.74 \\
(23)\end{array}$ & $\begin{array}{l}3.50 \\
(22)\end{array}$ & $\begin{array}{l}3.50 \\
(26)\end{array}$ & .698 & .595 \\
\hline $\begin{array}{l}\text { Park managers do not } \\
\text { have adequate } \\
\text { knowledge of climbing to } \\
\text { properly manage it at the } \\
\text { NRG. }\end{array}$ & $\begin{array}{l}3.31 \\
(16)\end{array}$ & $\begin{array}{l}3.08 \\
(25)\end{array}$ & $\begin{array}{l}3.53 \\
(15)\end{array}$ & $\begin{array}{l}3.13 \\
(15)\end{array}$ & $\begin{array}{l}3.15 \\
(20)\end{array}$ & .582 & .676 \\
\hline $\begin{array}{l}\text { Climber's practice of } \\
\text { land stewardship is not } \\
\text { adequate. }\end{array}$ & $\begin{array}{l}2.71 \\
(21)\end{array}$ & $\begin{array}{l}2.39 \\
(31)\end{array}$ & $\begin{array}{l}2.56 \\
(18)\end{array}$ & $\begin{array}{l}2.86 \\
(22)\end{array}$ & $\begin{array}{l}2.73 \\
(26)\end{array}$ & .835 & .506 \\
\hline
\end{tabular}




\begin{tabular}{|c|c|c|c|c|c|c|c|}
\hline $\begin{array}{l}\text { Poor communication of } \\
\text { rules and regulations is a } \\
\text { problem at NRG. }\end{array}$ & $\begin{array}{l}3.20 \\
(20)\end{array}$ & $\begin{array}{l}2.79 \\
(29)\end{array}$ & $\begin{array}{l}2.50 \\
(16)\end{array}$ & $\begin{array}{l}3.00 \\
(19)\end{array}$ & $\begin{array}{l}3.27 \\
(22)\end{array}$ & 2.254 & .068 \\
\hline $\begin{array}{l}\text { Climbers have a negative } \\
\text { attitude toward } \\
\text { management at the NRG. }\end{array}$ & $\begin{array}{l}2.67 \\
(15)\end{array}$ & $\begin{array}{l}2.72 \\
(25)\end{array}$ & $\begin{array}{l}3.06 \\
(16)\end{array}$ & $\begin{array}{l}2.53 \\
(15)\end{array}$ & $\begin{array}{l}2.70 \\
(23)\end{array}$ & .493 & .741 \\
\hline
\end{tabular}
${ }^{*} \mathrm{p}<0.050$

\section{RQ4: Do climbers of different climbing abilities differ in their attitudes toward cliff}

\section{resource management?}

As with the previous research question, a one-way analysis of variance was used to test for significant differences in low impact behaviors and attitudes toward cliff resource management among climbers of different abilities. Post-hoc comparisons with Tukey's test revealed some differences among climbers of different abilities. Climbers were asked to rate their level of experience with rock climbing and provide the grade of the most difficult climb they had accomplished. These results were used to divide respondents into three groups: novice, intermediate, and advanced/expert (due to the small number of experts they were grouped with the advanced climbers for analysis) (see Table 13). While these group sizes may appear small, VanVoorhis and Morgan (2007) determined that a group size of 7 is the lowest acceptable number for a one-way ANOVA in their Rules of Thumb for common statistical tests. Table 14. Ability of Climbers

\begin{tabular}{c|c} 
Ability & $\begin{array}{c}\text { Percent } \\
\text { (N) }\end{array}$ \\
\hline Novice & 11.9 \\
& $(18)$ \\
\hline Intermediate & 43.7 \\
& $(66)$ \\
\hline Advanced & 44.4 \\
& $(67)$ \\
\hline
\end{tabular}


Climbers of different climbing abilities were found to differ significantly in their attitudes toward cliff resource management (see Tables 15-16). Again, most of these differences occurred within the management statements, but two significant differences occurred within the low impact behavior statements (see Table 15). Post-hoc comparisons with Tukey's test revealed statistically significant differences between advanced/expert climbers and novice climbers. Statistically, advanced/expert climbers ( $\mathrm{M}=4.89)$ agreed that they "always used official trails" more than novice climbers $(M=4.78)$. Advanced/expert climbers $(M=4.71)$ also avoided trails that had been closed significantly more than novice climbers $(M=4.12)$.

Table 15. Low Impact Behavior Attitudes by Climbing Ability

Means $\quad$ F $\quad$ p

\begin{tabular}{|c|c|c|c|c|c|}
\hline & \multicolumn{3}{|c|}{ (N) } & & \\
\hline & Novice & Intermediate & $\begin{array}{l}\text { Advanced/ } \\
\text { Expert }\end{array}$ & & \\
\hline I always use official trails. & $\begin{array}{l}3.89 \mathrm{a} \\
(18)\end{array}$ & $\begin{array}{c}4.32 \mathrm{ab} \\
(65)\end{array}$ & $\begin{array}{c}4.53 \mathrm{~b} \\
(66)\end{array}$ & 4.448 & $.013^{*}$ \\
\hline $\begin{array}{l}\text { I avoid trails that have been } \\
\text { closed. }\end{array}$ & $\begin{array}{l}4.12 \mathrm{a} \\
(17)\end{array}$ & $\begin{array}{c}4.52 \mathrm{ab} \\
(66)\end{array}$ & $\begin{array}{l}4.71 \mathrm{~b} \\
(66)\end{array}$ & 5.215 & $.006^{*}$ \\
\hline $\begin{array}{l}\text { Fixed anchors should be } \\
\text { used to help preserve the } \\
\text { environment. }\end{array}$ & $\begin{array}{l}4.31 \mathrm{a} \\
(16)\end{array}$ & $\begin{array}{l}4.71 \mathrm{~b} \\
(66)\end{array}$ & $\begin{array}{l}4.71 \mathrm{~b} \\
(65)\end{array}$ & 3.095 & $.048^{*}$ \\
\hline $\begin{array}{l}\text { Permanent anchors provide } \\
\text { easy access to routes. }\end{array}$ & $\begin{array}{l}4.50 \mathrm{a} \\
(18)\end{array}$ & $\begin{array}{c}4.74 b \\
(66)\end{array}$ & $\begin{array}{c}4.39 \mathrm{ab} \\
(66)\end{array}$ & 4.232 & $.016^{*}$ \\
\hline $\begin{array}{l}\text { I park my vehicle in } \\
\text { designated areas. }\end{array}$ & $\begin{array}{l}4.44 a b \\
(18)\end{array}$ & $\begin{array}{c}4.77 \mathrm{a} \\
(65)\end{array}$ & $\begin{array}{c}4.73 b \\
(66)\end{array}$ & 3.157 & $.045^{*}$ \\
\hline $\begin{array}{l}\text { Chiseling holds is an } \\
\text { acceptable practice. }\end{array}$ & $\begin{array}{l}1.91 \mathrm{a} \\
(11)\end{array}$ & $\begin{array}{c}1.41 \mathrm{ab} \\
(63)\end{array}$ & $\begin{array}{c}1.25 \mathrm{~b} \\
(65)\end{array}$ & 3.841 & $.024^{*}$ \\
\hline $\begin{array}{l}\text { Permanent anchors are not } \\
\text { needed. }\end{array}$ & $\begin{array}{c}2.64 \mathrm{ab} \\
(14)\end{array}$ & $\begin{array}{c}1.60 \mathrm{a} \\
(65)\end{array}$ & $\begin{array}{c}1.80 \mathrm{~b} \\
(66)\end{array}$ & 9.547 & $.000^{*}$ \\
\hline I always pack out my trash. & $\begin{array}{l}4.78 \\
(18)\end{array}$ & $\begin{array}{l}4.91 \\
(66)\end{array}$ & $\begin{array}{l}4.89 \\
(66)\end{array}$ & .596 & .552 \\
\hline $\begin{array}{l}\text { I respect revegetation } \\
\text { efforts. }\end{array}$ & $\begin{array}{l}4.59 \\
(17)\end{array}$ & $\begin{array}{l}4.62 \\
(66)\end{array}$ & $\begin{array}{l}4.82 \\
(65)\end{array}$ & 3.041 & .051 \\
\hline $\begin{array}{l}\text { I practice Leave No Trace } \\
\text { when I hike/climb. }\end{array}$ & $\begin{array}{l}4.47 \\
(17)\end{array}$ & $\begin{array}{l}4.80 \\
(65)\end{array}$ & $\begin{array}{l}4.77 \\
(66)\end{array}$ & 2.890 & .059 \\
\hline
\end{tabular}




\begin{tabular}{|c|c|c|c|c|c|}
\hline $\begin{array}{l}\text { I try to keep my noise level } \\
\text { down. }\end{array}$ & $\begin{array}{l}4.22 \\
(18)\end{array}$ & $\begin{array}{l}4.42 \\
(66)\end{array}$ & $\begin{array}{l}4.45 \\
(66)\end{array}$ & .700 & .498 \\
\hline $\begin{array}{l}\text { I comply with local climbing } \\
\text { regulations. }\end{array}$ & $\begin{array}{l}4.44 \\
(16)\end{array}$ & $\begin{array}{l}4.74 \\
(65)\end{array}$ & $\begin{array}{l}4.71 \\
(66)\end{array}$ & 1.967 & .144 \\
\hline $\begin{array}{l}\text { Old gear should be removed } \\
\text { from belay and rappel sites. }\end{array}$ & $\begin{array}{l}4.67 \\
(18)\end{array}$ & $\begin{array}{l}4.60 \\
(65)\end{array}$ & $\begin{array}{l}4.41 \\
(66)\end{array}$ & 1.693 & .188 \\
\hline $\begin{array}{l}\text { I always consider the local } \\
\text { area ethics when I climb. }\end{array}$ & $\begin{array}{l}4.47 \\
(17)\end{array}$ & $\begin{array}{l}4.68 \\
(65)\end{array}$ & $\begin{array}{l}4.68 \\
(66)\end{array}$ & 1.064 & .348 \\
\hline $\begin{array}{l}\text { Permanent bolted anchors } \\
\text { should be used at the top of } \\
\text { climbs. }\end{array}$ & $\begin{array}{l}4.39 \\
(18)\end{array}$ & $\begin{array}{l}4.56 \\
(66)\end{array}$ & $\begin{array}{l}4.39 \\
(66)\end{array}$ & .977 & .379 \\
\hline $\begin{array}{l}\text { It is ok to remove/clean } \\
\text { lichens. }\end{array}$ & $\begin{array}{l}2.43 \\
(14)\end{array}$ & $\begin{array}{l}2.94 \\
(64)\end{array}$ & $\begin{array}{l}2.92 \\
(65)\end{array}$ & 1.127 & .327 \\
\hline $\begin{array}{l}\text { It is ok to remove/clean } \\
\text { mosses and other vegetation. }\end{array}$ & $\begin{array}{l}2.20 \\
(15)\end{array}$ & $\begin{array}{l}2.80 \\
(64)\end{array}$ & $\begin{array}{l}2.71 \\
(65)\end{array}$ & 1.487 & .230 \\
\hline $\begin{array}{l}\text { It is ok to secure top ropes } \\
\text { to trees. }\end{array}$ & $\begin{array}{l}3.35 \\
(17)\end{array}$ & $\begin{array}{l}3.11 \\
(66)\end{array}$ & $\begin{array}{l}2.95 \\
(65)\end{array}$ & .846 & .431 \\
\hline $\begin{array}{l}\text { Permanent anchors increase } \\
\text { the use of routes too much. }\end{array}$ & $\begin{array}{l}2.58 \\
(12)\end{array}$ & $\begin{array}{l}2.37 \\
(65)\end{array}$ & $\begin{array}{l}2.23 \\
(66)\end{array}$ & .780 & .460 \\
\hline $\begin{array}{l}\text { Soils and plants along the } \\
\text { park trails are hardy and } \\
\text { not easily damaged. }\end{array}$ & $\begin{array}{l}2.25 \\
(16)\end{array}$ & $\begin{array}{l}2.37 \\
(59)\end{array}$ & $\begin{array}{l}2.18 \\
(65)\end{array}$ & .672 & .512 \\
\hline $\begin{array}{l}\text { I respect seasonal closures at } \\
\text { certain crags for wildlife } \\
\text { needs. }\end{array}$ & $\begin{array}{l}4.33 \\
(15)\end{array}$ & $\begin{array}{l}4.49 \\
(65)\end{array}$ & $\begin{array}{l}4.35 \\
(66)\end{array}$ & .601 & .550 \\
\hline $\begin{array}{l}\text { The park provides clear } \\
\text { indications about which } \\
\text { trails should not be used. }\end{array}$ & $\begin{array}{l}3.14 \\
(14)\end{array}$ & $\begin{array}{l}3.27 \\
(63)\end{array}$ & $\begin{array}{l}3.57 \\
(65)\end{array}$ & 1.672 & .192 \\
\hline $\begin{array}{l}\text { Compared to other people I } \\
\text { saw on this visit, I am } \\
\text { causing less damage to the } \\
\text { soils and vegetation. }\end{array}$ & $\begin{array}{l}3.54 \\
(13)\end{array}$ & $\begin{array}{l}3.45 \\
(60)\end{array}$ & $\begin{array}{l}3.57 \\
(61)\end{array}$ & .372 & .690 \\
\hline $\begin{array}{l}\text { I feel a responsibility to help } \\
\text { protect the plants and } \\
\text { ecosystems in the park. }\end{array}$ & $\begin{array}{l}4.35 \\
(17)\end{array}$ & $\begin{array}{l}4.38 \\
(65)\end{array}$ & $\begin{array}{l}4.28 \\
(65)\end{array}$ & .321 & .726 \\
\hline $\begin{array}{l}\text { The continued use of } \\
\text { unofficial visitor created } \\
\text { trails is a serious threat to } \\
\text { plants and soils along the } \\
\text { park trails. }\end{array}$ & $\begin{array}{l}3.79 \\
(14)\end{array}$ & $\begin{array}{l}3.65 \\
(63)\end{array}$ & $\begin{array}{l}3.73 \\
(62)\end{array}$ & .122 & .885 \\
\hline
\end{tabular}




\begin{tabular}{|l|l|l|l|l|l|}
\hline $\begin{array}{l}\text { It is ok to walk off the } \\
\text { official trails, if I stay on }\end{array}$ & $\begin{array}{l}2.73 \\
(15)\end{array}$ & $\begin{array}{l}2.44 \\
(64)\end{array}$ & $\begin{array}{l}2.31 \\
(65)\end{array}$ & 1.062 & .349 \\
$\begin{array}{l}\text { unofficial trails already } \\
\text { created by visitors. }\end{array}$ & & & & & \\
\hline
\end{tabular}
$* \mathrm{p}<0.050$

Post-hoc comparisons with Tukey's test also revealed some statistically significant differences between climbers of different abilities with regards to management of cliff resources (Table 16). Within the management statements, novice climbers $(M=4.31)$ differed significantly from intermediate $(M=4.71) \&$ advanced $(M=4.71)$ climbers in regards to whether fixed anchors should be used to preserve the environment. Similarly, intermediate climbers $(M=4.74)$ differ significantly from advanced/expert climbers $(M=4.39)$ in regards to permanent anchors providing easy access to routes. Novice climbers $(\mathrm{M}=2.64)$ differed significantly from intermediate climbers $(M=1.60)$ and advanced/expert climbers $(M=1.80)$ as to whether permanent anchors were needed or not. Novice $(\mathrm{M}=1.91)$ and advanced/expert climbers $(\mathrm{M}=1.25)$ differed significantly on whether chiseling holds in an acceptable practice. Intermediate climbers $(M=4.77)$ differed significantly from novice climbers $(M=4.44)$ as to whether they always parked their vehicles in designated areas. Advanced/expert climbers $(M=4.18)$ differed significantly from novice climbers $(M=3.53)$ in their agreement that the impact of bolts and anchors on the environment is minimal. Novice $(\mathrm{M}=2.69)$, intermediate $(M=3.59)$, and advanced/expert climbers $(M=4.17)$ all differed significantly on their familiarity with climbing regulations - the more skilled the climber, the more familiar they were with regulations. Intermediate and Novice climbers (both $\mathrm{M}=3.65$ ) differed significantly from advanced/expert climbers $(\mathrm{M}=3.16)$ in their agreement with the statement "I trust that the NPS managers at NERI will do what is right for the park".

Table 16. Management Attitudes by Climbing Ability 
(N)

\begin{tabular}{|c|c|c|c|c|c|}
\hline & & & \\
\hline & Novice & Intermediate & $\begin{array}{c}\text { Advanced } \\
\text { /Expert }\end{array}$ & & \\
\hline $\begin{array}{l}\text { The impact of bolts and } \\
\text { anchors on the natural } \\
\text { resource is minimal. }\end{array}$ & $\begin{array}{c}3.53 \mathrm{ab} \\
(17)\end{array}$ & $\begin{array}{c}4.02 \mathrm{a} \\
(65)\end{array}$ & $\begin{array}{c}4.18 \mathrm{~b} \\
(66)\end{array}$ & 3.782 & $.025^{*}$ \\
\hline $\begin{array}{l}\text { I am familiar with the climbing } \\
\text { regulations at the NRG. }\end{array}$ & $\begin{array}{l}2.69 \mathrm{a} \\
(16)\end{array}$ & $\begin{array}{l}3.59 \mathrm{ab} \\
(61)\end{array}$ & $\begin{array}{l}4.17 \mathrm{~b} \\
(64)\end{array}$ & 17.640 & $.000^{*}$ \\
\hline $\begin{array}{l}\text { I trust that the NPS managers } \\
\text { at the NRG will do what is } \\
\text { right for the park. }\end{array}$ & $\begin{array}{l}3.65 \mathrm{a} \\
(17)\end{array}$ & $\begin{array}{c}3.65 \mathrm{a} \\
(55)\end{array}$ & $\begin{array}{c}3.16 \mathrm{~b} \\
(57)\end{array}$ & 3.560 & $.031^{*}$ \\
\hline $\begin{array}{l}\text { There should be official } \\
\text { regulations concerning where, } \\
\text { when, and how bolts and fixed } \\
\text { anchors should be used. }\end{array}$ & $\begin{array}{l}4.12 \\
(17)\end{array}$ & $\begin{array}{l}3.53 \\
(64)\end{array}$ & $\begin{array}{l}3.62 \\
(65)\end{array}$ & 2.049 & .133 \\
\hline $\begin{array}{l}\text { The presence of numerous } \\
\text { bolts and anchors negatively } \\
\text { affects my climbing } \\
\text { experiences. }\end{array}$ & $\begin{array}{l}2.18 \\
(17)\end{array}$ & $\begin{array}{l}1.83 \\
(66)\end{array}$ & $\begin{array}{l}1.89 \\
(66)\end{array}$ & .925 & .399 \\
\hline $\begin{array}{l}\text { The NPS managers at the NRG } \\
\text { share my values. }\end{array}$ & $\begin{array}{l}3.40 \\
(10)\end{array}$ & $\begin{array}{l}3.17 \\
(46)\end{array}$ & $\begin{array}{l}3.10 \\
(40)\end{array}$ & .408 & .666 \\
\hline $\begin{array}{l}\text { All climbing guide services } \\
\text { should be required to be } \\
\text { certified or accredited. }\end{array}$ & $\begin{array}{l}4.59 \\
(17)\end{array}$ & $\begin{array}{l}4.22 \\
(63)\end{array}$ & $\begin{array}{l}4.15 \\
(65)\end{array}$ & 1.303 & .275 \\
\hline $\begin{array}{l}\text { Climbing is not treated fairly in } \\
\text { the management process when } \\
\text { compared to other recreation } \\
\text { activities at the NRG. }\end{array}$ & $\begin{array}{c}2.88 \\
(8)\end{array}$ & $\begin{array}{l}3.07 \\
(45)\end{array}$ & $\begin{array}{l}3.27 \\
(48)\end{array}$ & 910 & .406 \\
\hline $\begin{array}{l}\text { Climbing group sizes should be } \\
\text { limited at NRG. }\end{array}$ & $\begin{array}{l}3.31 \\
(16)\end{array}$ & $\begin{array}{l}2.97 \\
(62)\end{array}$ & $\begin{array}{l}3.06 \\
(66)\end{array}$ & .447 & .640 \\
\hline $\begin{array}{l}\text { Other recreational groups are } \\
\text { offended by seeing climbing } \\
\text { bolts or anchors. }\end{array}$ & $\begin{array}{l}2.08 \\
(12)\end{array}$ & $\begin{array}{l}2.04 \\
(47)\end{array}$ & $\begin{array}{l}1.88 \\
(52)\end{array}$ & .478 & .622 \\
\hline $\begin{array}{l}\text { Climbers need to be educated } \\
\text { concerning their role in the } \\
\text { management process. }\end{array}$ & $\begin{array}{l}4.18 \\
(17)\end{array}$ & $\begin{array}{l}4.11 \\
(63)\end{array}$ & $\begin{array}{l}4.12 \\
(65)\end{array}$ & .047 & .954 \\
\hline $\begin{array}{l}\text { Large groups of climbers } \\
\text { detracted from my experience } \\
\text { at NRG. }\end{array}$ & $\begin{array}{l}2.41 \\
(17)\end{array}$ & $\begin{array}{l}2.92 \\
(65)\end{array}$ & $\begin{array}{l}2.83 \\
(66)\end{array}$ & 1.256 & .288 \\
\hline Most climbing areas need an & 3.65 & 3.43 & 3.44 & .289 & .750 \\
\hline
\end{tabular}




\begin{tabular}{|c|c|c|c|c|c|}
\hline $\begin{array}{l}\text { official management plan in } \\
\text { order to provide sustained use. }\end{array}$ & (17) & $(60)$ & (63) & & \\
\hline $\begin{array}{l}\text { The NPS managers at the NRG } \\
\text { have the same goals as me. }\end{array}$ & $\begin{array}{c}3.13 \\
(8)\end{array}$ & $\begin{array}{l}3.24 \\
(41)\end{array}$ & $\begin{array}{l}2.71 \\
(42)\end{array}$ & 3.025 & .054 \\
\hline $\begin{array}{l}\text { Park managers do not have } \\
\text { adequate knowledge of } \\
\text { climbing to properly manage it } \\
\text { at the NRG. }\end{array}$ & $\begin{array}{c}3.11 \\
(9)\end{array}$ & $\begin{array}{l}3.26 \\
(46)\end{array}$ & $\begin{array}{l}3.16 \\
(45)\end{array}$ & .168 & .846 \\
\hline $\begin{array}{l}\text { Climber's practice of land } \\
\text { stewardship is not adequate. }\end{array}$ & $\begin{array}{l}2.93 \\
(15)\end{array}$ & $\begin{array}{l}2.56 \\
(54)\end{array}$ & $\begin{array}{l}2.56 \\
(59)\end{array}$ & .894 & .412 \\
\hline $\begin{array}{l}\text { Poor communication of rules } \\
\text { and regulations is a problem at } \\
\text { NRG. }\end{array}$ & $\begin{array}{l}3.15 \\
(13)\end{array}$ & $\begin{array}{l}3.04 \\
(49)\end{array}$ & $\begin{array}{l}2.91 \\
(54)\end{array}$ & .458 & .634 \\
\hline $\begin{array}{l}\text { Climbers have a negative } \\
\text { attitude toward management at } \\
\text { the NRG. }\end{array}$ & $\begin{array}{r}2.67 \\
(9)\end{array}$ & $\begin{array}{l}2.67 \\
(42)\end{array}$ & $\begin{array}{l}2.74 \\
(50)\end{array}$ & .056 & .946 \\
\hline
\end{tabular}

RQ5: Does where (indoors vs. outdoors) climbers learn to climb influence their attitudes

\section{toward cliff resource management?}

Climbers were asked where they learned to climb ("mostly inside", "mostly outside", or “evenly split") and a one-way ANOVA was used to analyze the differences between the three groups in regards to low impact behaviors and cliff resource management. Post-hoc comparisons with Tukey's test revealed a couple of differences between climbers who learned to climb in different environments.

Table 17. Climbers Segmented by Learning Environment Learning Environment

Mostly Inside (At a Rock Gym)

Pretty Evenly Split Mostly Outside

Percent 24.5 34.4 41.1

The only significant differences between these three groups occurred in regards to the use of permanent anchors and familiarity with climbing regulations (see Tables 18-19). Within the low impact statements, climbers who learned to climb mostly inside $(M=4.73)$ reported higher 
levels of agreement with the statement that "permanent bolted anchors should be used at the top of climbs" than climbers who learned to climb mostly outside $(\mathrm{M}=4.33)$. Within the management statements, climbers who learned to climb mostly outside $(\mathrm{M}=3.91)$ were more familiar with climbing regulations in NERI than climbers who learned to climb mostly inside $(\mathrm{M}=3.38)$.

Table 18. Low Impact Behavior Attitudes by Learning Environment

\begin{tabular}{|c|c|c|c|c|c|}
\hline & \multicolumn{3}{|c|}{ Means } & \multirow[t]{2}{*}{ F } & \multirow[t]{2}{*}{$\mathbf{p}$} \\
\hline & $\begin{array}{l}\text { Mostly } \\
\text { inside }\end{array}$ & $\begin{array}{l}\text { Evenly } \\
\text { split }\end{array}$ & $\begin{array}{l}\text { Mostly } \\
\text { outside }\end{array}$ & & \\
\hline $\begin{array}{l}\text { Permanent bolted anchors should } \\
\text { be used at the top of climbs. }\end{array}$ & $\begin{array}{c}4.73 \mathrm{a} \\
(37)\end{array}$ & $\begin{array}{c}4.42 \mathrm{ab} \\
(52)\end{array}$ & $\begin{array}{c}4.33 b \\
(61)\end{array}$ & 3.730 & $.026^{*}$ \\
\hline I always pack out my trash. & $\begin{array}{l}4.89 \\
(37)\end{array}$ & $\begin{array}{l}4.85 \\
(52)\end{array}$ & $\begin{array}{l}4.93 \\
(61)\end{array}$ & .535 & .587 \\
\hline I always use official trails. & $\begin{array}{l}4.35 \\
(37)\end{array}$ & $\begin{array}{l}4.43 \\
(51)\end{array}$ & $\begin{array}{l}4.33 \\
(61)\end{array}$ & .219 & .804 \\
\hline I respect revegetation efforts. & $\begin{array}{l}4.64 \\
(36)\end{array}$ & $\begin{array}{l}4.71 \\
(52)\end{array}$ & $\begin{array}{l}4.75 \\
(60)\end{array}$ & .557 & .574 \\
\hline I avoid trails that have been closed. & $\begin{array}{l}4.51 \\
(37)\end{array}$ & $\begin{array}{l}4.73 \\
(52)\end{array}$ & $\begin{array}{l}4.45 \\
(60)\end{array}$ & 2.342 & .100 \\
\hline $\begin{array}{l}\text { I practice Leave No Trace when I } \\
\text { hike/climb. }\end{array}$ & $\begin{array}{l}4.81 \\
(37)\end{array}$ & $\begin{array}{l}4.71 \\
(51)\end{array}$ & $\begin{array}{l}4.77 \\
(60)\end{array}$ & .457 & .634 \\
\hline I try to keep my noise level down. & $\begin{array}{l}4.27 \\
(37)\end{array}$ & $\begin{array}{l}4.46 \\
(52)\end{array}$ & $\begin{array}{l}4.44 \\
(61)\end{array}$ & .815 & .444 \\
\hline $\begin{array}{l}\text { Fixed anchors should be used to } \\
\text { help preserve the environment. }\end{array}$ & $\begin{array}{l}4.62 \\
(37)\end{array}$ & $\begin{array}{l}4.71 \\
(51)\end{array}$ & $\begin{array}{l}4.63 \\
(59)\end{array}$ & .276 & .759 \\
\hline $\begin{array}{l}\text { I comply with local climbing } \\
\text { regulations. }\end{array}$ & $\begin{array}{l}4.76 \\
(37)\end{array}$ & $\begin{array}{l}4.73 \\
(51)\end{array}$ & $\begin{array}{l}4.64 \\
(59)\end{array}$ & .545 & .581 \\
\hline $\begin{array}{l}\text { Old gear should be removed from } \\
\text { belay and rappel sites. }\end{array}$ & $\begin{array}{l}4.69 \\
(36)\end{array}$ & $\begin{array}{l}4.50 \\
(52)\end{array}$ & $\begin{array}{l}4.41 \\
(61)\end{array}$ & 1.883 & .156 \\
\hline $\begin{array}{l}\text { Permanent anchors provide easy } \\
\text { access to routes. }\end{array}$ & $\begin{array}{l}4.68 \\
(37)\end{array}$ & $\begin{array}{l}4.40 \\
(52)\end{array}$ & $\begin{array}{l}4.62 \\
(61)\end{array}$ & 2.020 & .136 \\
\hline $\begin{array}{l}\text { I park my vehicle in designated } \\
\text { areas. }\end{array}$ & $\begin{array}{l}4.61 \\
(36)\end{array}$ & $\begin{array}{l}4.81 \\
(52)\end{array}$ & $\begin{array}{l}4.69 \\
(61)\end{array}$ & 1.791 & .170 \\
\hline $\begin{array}{l}\text { I always consider the local area } \\
\text { ethics when I climb. }\end{array}$ & $\begin{array}{l}4.59 \\
(37)\end{array}$ & $\begin{array}{l}4.67 \\
(51)\end{array}$ & $\begin{array}{l}4.68 \\
(60)\end{array}$ & .305 & .738 \\
\hline It is ok to remove/clean lichens. & $\begin{array}{l}2.97 \\
(36)\end{array}$ & $\begin{array}{l}2.86 \\
(51)\end{array}$ & $\begin{array}{l}2.86 \\
(56)\end{array}$ & .120 & .887 \\
\hline
\end{tabular}




\begin{tabular}{|c|c|c|c|c|c|}
\hline $\begin{array}{l}\text { It is ok to remove/clean mosses and } \\
\text { other vegetation. }\end{array}$ & $\begin{array}{l}2.70 \\
(37)\end{array}$ & $\begin{array}{l}2.59 \\
(49)\end{array}$ & $\begin{array}{l}2.79 \\
(58)\end{array}$ & .363 & .696 \\
\hline $\begin{array}{l}\text { Chiseling holds is an acceptable } \\
\text { practice. }\end{array}$ & $\begin{array}{l}1.56 \\
(36)\end{array}$ & $\begin{array}{l}1.22 \\
(49)\end{array}$ & $\begin{array}{l}1.38 \\
(55)\end{array}$ & 1.996 & .140 \\
\hline It is ok to secure top ropes to trees. & $\begin{array}{l}2.81 \\
(37)\end{array}$ & $\begin{array}{l}3.20 \\
(51)\end{array}$ & $\begin{array}{l}3.12 \\
(60)\end{array}$ & 1.255 & .288 \\
\hline $\begin{array}{l}\text { Permanent anchors increase the } \\
\text { use of routes too much. }\end{array}$ & $\begin{array}{l}2.43 \\
(37)\end{array}$ & $\begin{array}{l}2.22 \\
(50)\end{array}$ & $\begin{array}{l}2.34 \\
(56)\end{array}$ & .493 & .612 \\
\hline $\begin{array}{l}\text { Soils and plants along the park } \\
\text { trails are hardy and not easily } \\
\text { damaged. }\end{array}$ & $\begin{array}{l}2.32 \\
(34)\end{array}$ & $\begin{array}{l}2.21 \\
(48)\end{array}$ & $\begin{array}{l}2.28 \\
(58)\end{array}$ & .171 & .843 \\
\hline $\begin{array}{l}\text { I respect seasonal closures at } \\
\text { certain crags for wildlife needs. }\end{array}$ & $\begin{array}{l}4.28 \\
(36)\end{array}$ & $\begin{array}{l}4.53 \\
(51)\end{array}$ & $\begin{array}{l}4.41 \\
(59)\end{array}$ & 1.046 & .354 \\
\hline $\begin{array}{l}\text { The park provides clear } \\
\text { indications about which trails } \\
\text { should not be used. }\end{array}$ & $\begin{array}{l}3.32 \\
(34)\end{array}$ & $\begin{array}{l}3.29 \\
(49)\end{array}$ & $\begin{array}{l}3.53 \\
(58)\end{array}$ & .804 & .450 \\
\hline $\begin{array}{l}\text { Compared to other people I saw on } \\
\text { this visit, I am causing less damage } \\
\text { to the soils and vegetation. }\end{array}$ & $\begin{array}{l}3.38 \\
(32)\end{array}$ & $\begin{array}{l}3.64 \\
(45)\end{array}$ & $\begin{array}{l}3.53 \\
(57)\end{array}$ & 1.059 & .350 \\
\hline $\begin{array}{l}\text { Permanent anchors are not } \\
\text { needed. }\end{array}$ & $\begin{array}{l}1.79 \\
(34)\end{array}$ & $\begin{array}{l}1.80 \\
(51)\end{array}$ & $\begin{array}{l}1.80 \\
(60)\end{array}$ & .001 & .999 \\
\hline $\begin{array}{l}\text { I feel a responsibility to help } \\
\text { protect the plants and ecosystems } \\
\text { in the park. }\end{array}$ & $\begin{array}{l}4.33 \\
(36)\end{array}$ & $\begin{array}{l}4.18 \\
(50)\end{array}$ & $\begin{array}{l}4.46 \\
(61)\end{array}$ & 1.822 & .165 \\
\hline $\begin{array}{l}\text { The continued use of unofficial } \\
\text { visitor created trails is a serious } \\
\text { threat to plants and soils along the } \\
\text { park trails. }\end{array}$ & $\begin{array}{l}3.70 \\
(33)\end{array}$ & $\begin{array}{l}3.75 \\
(48)\end{array}$ & $\begin{array}{l}3.66 \\
(59)\end{array}$ & .087 & .917 \\
\hline $\begin{array}{l}\text { It is ok to walk off the official } \\
\text { trails, if I stay on unofficial trails } \\
\text { already created by visitors. }\end{array}$ & $\begin{array}{l}2.26 \\
(34)\end{array}$ & $\begin{array}{l}2.43 \\
(51)\end{array}$ & $\begin{array}{l}2.47 \\
(60)\end{array}$ & .430 & .652 \\
\hline
\end{tabular}

Table 19. Management Attitudes by Learning Environment

\begin{tabular}{l|c|c|c|c|c}
\cline { 2 - 5 } & $\begin{array}{c}\text { Mostly } \\
\text { inside }\end{array}$ & $\begin{array}{c}\text { Evenly } \\
\text { split }\end{array}$ & $\begin{array}{c}\text { Mostly } \\
\text { outside }\end{array}$ & F & \\
\hline I am familiar with the climbing & $3.38 \mathrm{a}$ & $3.86 \mathrm{ab}$ & $3.91 \mathrm{~b}$ & 3.225 & $.043^{*}$ \\
\hline
\end{tabular}




\begin{tabular}{|c|c|c|c|c|c|}
\hline regulations at the NRG. & (34) & $(50)$ & $(57)$ & & \\
\hline $\begin{array}{l}\text { The impact of bolts and anchors on } \\
\text { the natural resource is minimal. }\end{array}$ & $\begin{array}{l}4.14 \\
(37)\end{array}$ & $\begin{array}{l}4.04 \\
(51)\end{array}$ & $\begin{array}{l}3.95 \\
(60)\end{array}$ & .493 & .612 \\
\hline $\begin{array}{l}\text { There should be official } \\
\text { regulations concerning where, } \\
\text { when, and how bolts and fixed } \\
\text { anchors should be used. }\end{array}$ & $\begin{array}{l}3.89 \\
(37)\end{array}$ & $\begin{array}{l}3.63 \\
(49)\end{array}$ & $\begin{array}{l}3.48 \\
(60)\end{array}$ & 1.667 & .193 \\
\hline $\begin{array}{l}\text { The presence of numerous bolts } \\
\text { and anchors negatively affects my } \\
\text { climbing experiences. }\end{array}$ & $\begin{array}{l}1.78 \\
(37)\end{array}$ & $\begin{array}{l}1.87 \\
(52)\end{array}$ & $\begin{array}{l}2.00 \\
(60)\end{array}$ & .671 & .513 \\
\hline $\begin{array}{l}\text { The NPS managers at the NRG } \\
\text { share my values. }\end{array}$ & $\begin{array}{l}3.29 \\
(21)\end{array}$ & $\begin{array}{l}3.27 \\
(30)\end{array}$ & $\begin{array}{l}3.02 \\
(45)\end{array}$ & .875 & .420 \\
\hline $\begin{array}{l}\text { All climbing guide services should } \\
\text { be required to be certified or } \\
\text { accredited. }\end{array}$ & $\begin{array}{l}4.36 \\
(36)\end{array}$ & $\begin{array}{l}4.22 \\
(50)\end{array}$ & $\begin{array}{l}4.14 \\
(59)\end{array}$ & .554 & .576 \\
\hline $\begin{array}{l}\text { Climbing is not treated fairly in } \\
\text { the management process when } \\
\text { compared to other recreation } \\
\text { activities at the NRG. }\end{array}$ & $\begin{array}{l}3.26 \\
(23)\end{array}$ & $\begin{array}{l}2.97 \\
(33)\end{array}$ & $\begin{array}{l}3.22 \\
(46)\end{array}$ & .887 & .415 \\
\hline $\begin{array}{l}\text { Climbing group sizes should be } \\
\text { limited at NRG. }\end{array}$ & $\begin{array}{l}2.81 \\
(36)\end{array}$ & $\begin{array}{l}2.96 \\
(50)\end{array}$ & $\begin{array}{l}3.29 \\
(58)\end{array}$ & 1.774 & .173 \\
\hline $\begin{array}{l}\text { Other recreational groups are } \\
\text { offended by seeing climbing bolts } \\
\text { or anchors. }\end{array}$ & $\begin{array}{l}2.07 \\
(29)\end{array}$ & $\begin{array}{l}1.97 \\
(36)\end{array}$ & $\begin{array}{l}1.91 \\
(45)\end{array}$ & .266 & .767 \\
\hline $\begin{array}{l}\text { I trust that the NPS managers at } \\
\text { the NRG will do what is right for } \\
\text { the park. }\end{array}$ & $\begin{array}{l}3.56 \\
(32)\end{array}$ & $\begin{array}{l}3.52 \\
(44)\end{array}$ & $\begin{array}{l}3.27 \\
(52)\end{array}$ & .998 & .372 \\
\hline $\begin{array}{l}\text { Climbers need to be educated } \\
\text { concerning their role in the } \\
\text { management process. }\end{array}$ & $\begin{array}{l}4.14 \\
(37)\end{array}$ & $\begin{array}{l}4.29 \\
(49)\end{array}$ & $\begin{array}{l}3.98 \\
(59)\end{array}$ & 2.095 & .127 \\
\hline $\begin{array}{l}\text { Large groups of climbers detracted } \\
\text { from my experience at NRG. }\end{array}$ & $\begin{array}{l}2.84 \\
(37)\end{array}$ & $\begin{array}{l}2.96 \\
(52)\end{array}$ & $\begin{array}{l}2.73 \\
(59)\end{array}$ & .525 & .593 \\
\hline $\begin{array}{l}\text { Most climbing areas need an } \\
\text { official management plan in order } \\
\text { to provide sustained use. }\end{array}$ & $\begin{array}{l}3.40 \\
(35)\end{array}$ & $\begin{array}{l}3.50 \\
(48)\end{array}$ & $\begin{array}{l}3.49 \\
(57)\end{array}$ & .106 & .899 \\
\hline $\begin{array}{l}\text { The NPS managers at the NRG } \\
\text { have the same goals as me. }\end{array}$ & $\begin{array}{l}3.04 \\
(24)\end{array}$ & $\begin{array}{l}3.21 \\
(38)\end{array}$ & $\begin{array}{l}2.79 \\
(39)\end{array}$ & 1.445 & .241 \\
\hline $\begin{array}{l}\text { Park managers do not have } \\
\text { adequate knowledge of climbing to } \\
\text { properly manage it at the NRG. }\end{array}$ & $\begin{array}{l}3.13 \\
(24)\end{array}$ & $\begin{array}{l}3.09 \\
(33)\end{array}$ & $\begin{array}{l}3.33 \\
(42)\end{array}$ & .646 & .526 \\
\hline
\end{tabular}




\begin{tabular}{|l|l|l|l|l|l|}
\hline Climber's practice of land & 2.64 & 2.78 & 2.42 & 1.530 & .221 \\
\hline stewardship is not adequate. & $(33)$ & $(46)$ & $(48)$ & & \\
\hline Poor communication of rules and & 3.03 & 3.07 & 2.91 & .338 & .714 \\
regulations is a problem at NRG. & $(31)$ & $(41)$ & $(43)$ & & \\
\hline Climbers have a negative attitude & 2.70 & 2.64 & 2.77 & .146 & .864 \\
\hline toward management at the NRG. & $(23)$ & $(33)$ & $(44)$ & & \\
\hline
\end{tabular}

$* \mathrm{p}<0.050$

RQ6: Does how (formally trained vs. informally) climbers learn to climb influence their attitudes toward cliff resource management?

Climbers were asked how they learned to climb and which method of learning had the most influence on their climbing practices. Based on their answers, they were determined to have learned to climb either informally (from family, friends, or a climbing club) or formally (from a college or university course or other formal course). Climbers who learned to climb formally made up $19 \%$ of the population, with 26 climbers. Climbers who learned to climb informally made up the remaining $81 \%$ of the sample, with 110 climbers, for a total of 136 climbers. Data were analyzed using a t-test to determine if there were any differences in attitudes toward cliff resource management and low impact behaviors based on the climber's learning experience. Some differences were found (Table 21-22).

Climbers who learned formally $(M=4.69)$ agreed more strongly than climbers who learned informally $(M=4.36)$ that they tried to keep their noise levels down. Climbers who learned informally $(M=2.40)$ reported higher levels of agreement than climbers who learned informally $(\mathrm{M}=1.83)$ with the statement "soils and plants along the trail are hardy and not easily damaged”. Climbers who learned formally $(\mathrm{M}=4.56)$ reported higher levels of agreement than climbers who learned informally $(\mathrm{M}=4.09)$ with the statement "climbing guide services should be certified or accredited". Climbers who learned formally $(\mathrm{M}=4.44)$ reported higher 
levels of agreement than climbers who learned informally $(\mathrm{M}=4.07)$ with the statement

"climbers need to be educated concerning their role in the management process".

Table 21. Low Impact Behavior Attitudes by Learning Style

\begin{tabular}{|c|c|c|c|c|}
\hline & \multicolumn{2}{|c|}{ Mean } & \multirow[t]{2}{*}{$t$} & \multirow[t]{2}{*}{$\mathbf{p}$} \\
\hline & Informal & Formal & & \\
\hline I try to keep my noise level down. & $\begin{array}{l}4.36 \\
(110)\end{array}$ & $\begin{array}{l}4.69 \\
(26)\end{array}$ & -2.504 & $.015^{*}$ \\
\hline $\begin{array}{l}\text { Soils and plants along the park trails are hardy } \\
\text { and not easily damaged. }\end{array}$ & $\begin{array}{l}2.40 \\
(102)\end{array}$ & $\begin{array}{l}1.83 \\
(24)\end{array}$ & 2.851 & $.005^{*}$ \\
\hline I always pack out my trash. & $\begin{array}{l}4.92 \\
(110)\end{array}$ & $\begin{array}{l}4.81 \\
(26)\end{array}$ & .689 & .497 \\
\hline I always use official trails. & $\begin{array}{l}4.30 \\
(109)\end{array}$ & $\begin{array}{l}4.58 \\
(26)\end{array}$ & -1.468 & .144 \\
\hline I respect revegetation efforts. & $\begin{array}{l}4.69 \\
(108)\end{array}$ & $\begin{array}{l}4.81 \\
(26)\end{array}$ & -1.135 & .263 \\
\hline I avoid trails that have been closed. & $\begin{array}{l}4.56 \\
(109)\end{array}$ & $\begin{array}{l}4.54 \\
(26)\end{array}$ & .134 & .893 \\
\hline I practice Leave No Trace when I hike/climb. & $\begin{array}{l}4.76 \\
(109)\end{array}$ & $\begin{array}{l}4.69 \\
(26)\end{array}$ & .598 & .551 \\
\hline $\begin{array}{l}\text { Fixed anchors should be used to help preserve } \\
\text { the environment. }\end{array}$ & $\begin{array}{l}4.64 \\
(108)\end{array}$ & $\begin{array}{l}4.68 \\
(25)\end{array}$ & -.288 & .774 \\
\hline I comply with local climbing regulations. & $\begin{array}{l}4.67 \\
(108)\end{array}$ & $\begin{array}{l}4.81 \\
(26)\end{array}$ & -1.447 & .154 \\
\hline $\begin{array}{l}\text { Old gear should be removed from belay and } \\
\text { rappel sites. }\end{array}$ & $\begin{array}{l}4.49 \\
(110)\end{array}$ & $\begin{array}{l}4.65 \\
(26)\end{array}$ & -1.054 & .294 \\
\hline $\begin{array}{l}\text { Permanent anchors provide easy access to } \\
\text { routes. }\end{array}$ & $\begin{array}{l}4.57 \\
(110)\end{array}$ & $\begin{array}{l}4.54 \\
(26)\end{array}$ & .218 & .827 \\
\hline I park my vehicle in designated areas. & $\begin{array}{l}4.70 \\
(109)\end{array}$ & $\begin{array}{l}4.77 \\
(26)\end{array}$ & -.656 & .513 \\
\hline $\begin{array}{l}\text { I always consider the local area ethics when I } \\
\text { climb. }\end{array}$ & $\begin{array}{l}4.67 \\
(108)\end{array}$ & $\begin{array}{l}4.73 \\
(26)\end{array}$ & -.539 & .591 \\
\hline $\begin{array}{l}\text { Permanent bolted anchors should be used at the } \\
\text { top of climbs. }\end{array}$ & $\begin{array}{l}4.43 \\
(110)\end{array}$ & $\begin{array}{l}4.42 \\
(26)\end{array}$ & .026 & .980 \\
\hline It is ok to remove/clean lichens. & $\begin{array}{l}2.96 \\
(107)\end{array}$ & $\begin{array}{l}2.91 \\
(22)\end{array}$ & .191 & .849 \\
\hline $\begin{array}{l}\text { It is ok to remove/clean mosses and other } \\
\text { vegetation. }\end{array}$ & $\begin{array}{l}2.79 \\
(107)\end{array}$ & $\begin{array}{l}2.52 \\
(23)\end{array}$ & .961 & .339 \\
\hline Chiseling holds is an acceptable practice. & 1.41 & 1.19 & 1.835 & .071 \\
\hline
\end{tabular}




\begin{tabular}{|c|c|c|c|c|}
\hline & $(105)$ & $(21)$ & & \\
\hline It is ok to secure top ropes to trees. & $\begin{array}{l}2.98 \\
(110)\end{array}$ & $\begin{array}{l}3.38 \\
(24)\end{array}$ & -1.464 & .146 \\
\hline $\begin{array}{l}\text { Permanent anchors increase the use of routes } \\
\text { too much. }\end{array}$ & $\begin{array}{c}2.33 \\
(107)\end{array}$ & $\begin{array}{l}2.27 \\
(22)\end{array}$ & .230 & .819 \\
\hline $\begin{array}{l}\text { I respect seasonal closures at certain crags for } \\
\text { wildlife needs. }\end{array}$ & $\begin{array}{l}4.45 \\
(110)\end{array}$ & $\begin{array}{l}4.52 \\
(23)\end{array}$ & -.404 & .687 \\
\hline $\begin{array}{l}\text { The park provides clear indications about which } \\
\text { trails should not be used. }\end{array}$ & $\begin{array}{l}3.45 \\
(105)\end{array}$ & $\begin{array}{l}3.35 \\
(23)\end{array}$ & .418 & .676 \\
\hline $\begin{array}{l}\text { Compared to other people I saw on this visit, I } \\
\text { am causing less damage to the soils and } \\
\text { vegetation. }\end{array}$ & $\begin{array}{l}3.44 \\
(101)\end{array}$ & $\begin{array}{l}3.74 \\
(23)\end{array}$ & -1.672 & .097 \\
\hline Permanent anchors are not needed. & $\begin{array}{c}1.74 \\
(107)\end{array}$ & $\begin{array}{l}2.00 \\
(25)\end{array}$ & -1.397 & .165 \\
\hline $\begin{array}{l}\text { I feel a responsibility to help protect the plants } \\
\text { and ecosystems in the park. }\end{array}$ & $\begin{array}{l}4.33 \\
(108)\end{array}$ & $\begin{array}{l}4.40 \\
(25)\end{array}$ & -.405 & .686 \\
\hline $\begin{array}{l}\text { The continued use of unofficial visitor created } \\
\text { trails is a serious threat to plants and soils along } \\
\text { the park trails. }\end{array}$ & $\begin{array}{c}3.63 \\
(103)\end{array}$ & $\begin{array}{l}3.92 \\
(24)\end{array}$ & -1.167 & .246 \\
\hline $\begin{array}{l}\text { It is ok to walk off the official trails, if I stay on } \\
\text { unofficial trails already created by visitors. }\end{array}$ & $\begin{array}{c}2.46 \\
(107)\end{array}$ & $\begin{array}{l}2.17 \\
(24)\end{array}$ & 1.247 & .215 \\
\hline
\end{tabular}
$* \mathrm{p}<0.050$

Table 22. Management Attitudes by Learning Style

Management Statements

\begin{tabular}{|c|c|c|c|}
\hline \multicolumn{2}{|c|}{ Mean } & \multirow[t]{2}{*}{$\mathbf{t}$} & \multirow[t]{2}{*}{$\mathbf{p}$} \\
\hline Informal & Formal & & \\
\hline $\begin{array}{c}4.09 \\
(107)\end{array}$ & $\begin{array}{l}4.56 \\
(25)\end{array}$ & -2.645 & $.011 *$ \\
\hline $\begin{array}{c}4.07 \\
(107)\end{array}$ & $\begin{array}{l}4.44 \\
(25)\end{array}$ & -2.224 & $.028 *$ \\
\hline $\begin{array}{c}4.06 \\
(109)\end{array}$ & $\begin{array}{l}3.72 \\
(25)\end{array}$ & 1.704 & .091 \\
\hline $\begin{array}{c}3.62 \\
(110)\end{array}$ & $\begin{array}{l}3.58 \\
(24)\end{array}$ & .144 & .886 \\
\hline $\begin{array}{c}1.84 \\
(110)\end{array}$ & $\begin{array}{l}1.96 \\
(25)\end{array}$ & -.603 & .548 \\
\hline $\begin{array}{l}3.18 \\
(72)\end{array}$ & $\begin{array}{l}3.28 \\
(18)\end{array}$ & -.396 & .693 \\
\hline
\end{tabular}




\begin{tabular}{|c|c|c|c|c|}
\hline $\begin{array}{l}\text { I am familiar with the climbing regulations at } \\
\text { the NRG. }\end{array}$ & $\begin{array}{l}3.75 \\
(106)\end{array}$ & $\begin{array}{l}3.70 \\
(23)\end{array}$ & .212 & .803 \\
\hline $\begin{array}{l}\text { Climbing is not treated fairly in the } \\
\text { management process when compared to other } \\
\text { recreation activities at the NRG. }\end{array}$ & $\begin{array}{l}3.17 \\
(76)\end{array}$ & $\begin{array}{l}2.79 \\
(19)\end{array}$ & 1.625 & .108 \\
\hline Climbing group sizes should be limited at NRG. & $\begin{array}{c}3.01 \\
(105)\end{array}$ & $\begin{array}{l}3.20 \\
(25)\end{array}$ & -.651 & .516 \\
\hline $\begin{array}{l}\text { Other recreational groups are offended by } \\
\text { seeing climbing bolts or anchors. }\end{array}$ & $\begin{array}{l}1.97 \\
(79)\end{array}$ & $\begin{array}{l}2.05 \\
(21)\end{array}$ & -.319 & .750 \\
\hline $\begin{array}{l}\text { I trust that the NPS managers at the NRG will } \\
\text { do what is right for the park. }\end{array}$ & $\begin{array}{l}3.39 \\
(95)\end{array}$ & $\begin{array}{l}3.71 \\
(24)\end{array}$ & -1.609 & .114 \\
\hline $\begin{array}{l}\text { Large groups of climbers detracted from my } \\
\text { experience at NRG. }\end{array}$ & $\begin{array}{l}2.79 \\
(109)\end{array}$ & $\begin{array}{l}3.24 \\
(25)\end{array}$ & -1.768 & .079 \\
\hline $\begin{array}{l}\text { Most climbing areas need an official } \\
\text { management plan in order to provide sustained } \\
\text { use. }\end{array}$ & $\begin{array}{c}3.38 \\
(104)\end{array}$ & $\begin{array}{l}3.84 \\
(25)\end{array}$ & -1.947 & .054 \\
\hline $\begin{array}{l}\text { The NPS managers at the NRG have the same } \\
\text { goals as me. }\end{array}$ & $\begin{array}{l}3.03 \\
(70)\end{array}$ & $\begin{array}{l}3.13 \\
(16)\end{array}$ & -.357 & .722 \\
\hline $\begin{array}{l}\text { Park managers do not have adequate knowledge } \\
\text { of climbing to properly manage it at the NRG. }\end{array}$ & $\begin{array}{l}3.20 \\
(74)\end{array}$ & $\begin{array}{l}3.28 \\
(18)\end{array}$ & -.304 & .762 \\
\hline $\begin{array}{l}\text { Climber's practice of land stewardship is not } \\
\text { adequate. }\end{array}$ & $\begin{array}{l}2.61 \\
(94)\end{array}$ & $\begin{array}{l}2.81 \\
(21)\end{array}$ & -.854 & .395 \\
\hline $\begin{array}{l}\text { Poor communication of rules and regulations is } \\
\text { a problem at NRG. }\end{array}$ & $\begin{array}{l}2.98 \\
(84)\end{array}$ & $\begin{array}{l}3.05 \\
(22)\end{array}$ & -.297 & .767 \\
\hline $\begin{array}{l}\text { Climbers have a negative attitude toward } \\
\text { management at the NRG. }\end{array}$ & $\begin{array}{l}2.76 \\
(75)\end{array}$ & $\begin{array}{l}2.44 \\
(18)\end{array}$ & 1.074 & .286 \\
\hline
\end{tabular}

${ }^{*} \mathrm{p}<0.050$

RQ7: Do hikers and climbers differ in who they trust for information on cliff resources?

Hikers and climbers were asked to rate how much they trusted eight different sources for information on cliff resources on a scale of 1 (strongly distrust) to 4 (strongly trust). An option for "neutral" or "don't know" was not provided. Smaldone and McKenney (2013) found that hikers and climbers in NERI vary greatly on who they most strongly trust for information about cliff resource management. Climbers were most likely to "strongly trust" climbing organizations 
and family and friends, and hikers were most likely to "strongly trust" NPS rangers in general and NPS rangers at NERI.

A Chi-square analysis was used in order to identify associations between levels of trust in sources of information and activity group (hikers and climbers), and a number of statistically significant relationships were found (See Table 23). Climbers and hikers differed significantly in their levels of trust for seven of the eight sources. Climbers were more likely than hikers to "strongly trust" friends and family. Hikers were more likely to "strongly trust" environmental organizations, while climbers were significantly more likely to "strongly trust" climbing organizations. Hikers were more than twice as likely to "strongly trust" scientists in general than climbers. Hikers were also more than three times more likely than climbers to "strongly trust" NPS rangers in general and NPS rangers at NERI. While both groups were distrustful of the mainstream news media, hikers were more likely than climbers to "somewhat trust" the media, and climbers were more likely than hikers to "strongly distrust" the media.

Table 23. Chi-square Analysis of Visitors' Trust of Cliff Management Resources

\begin{tabular}{l|c|c|l}
\hline Trust Scale & Hikers (N, \%) & Climbers (N,\%) & \\
\hline Friends and Family & $\mathrm{N}=151$ & $\mathrm{~N}=146$ & $x^{2}=12.336$ \\
\hline Strongly Trust & 40 & 65 & $\mathrm{df}=3$ \\
& $26.5 \%$ & $44.5 \%$ & $\mathrm{p}=.006$ \\
\hline Somewhat Trust & 86 & 69 & Cramer's \\
\hline Somewhat Distrust & $57.0 \%$ & $47.3 \%$ & $\mathrm{~V}=0.204$ \\
& 18 & 9 & \\
\hline Strongly Distrust & $11.9 \%$ & $6.2 \%$ & \\
& 7 & 3 & \\
\hline Environmental Organizations & $4.6 \%$ & $2.1 \%$ & \\
\hline Strongly Trust & $\mathrm{N}=151$ & $\mathrm{~N}=145$ & $x^{2}=18.788$ \\
& 65 & 37 & $\mathrm{df}=3$ \\
\hline Somewhat Trust & $43.0 \%$ & $25.5 \%$ & $\mathrm{p}=.000$ \\
& 78 & 86 & $\mathrm{Cramer}$ \\
\hline Somewhat Distrust & $51.7 \%$ & $59.3 \%$ & $\mathrm{~V}=0.252$ \\
& 3 & 18 & \\
\hline
\end{tabular}




\begin{tabular}{|c|c|c|c|}
\hline Strongly Distrust & $\begin{array}{c}5 \\
3.3 \%\end{array}$ & $\begin{array}{c}4 \\
2.8 \%\end{array}$ & \\
\hline Businesses & $\mathrm{N}=150$ & $\mathrm{~N}=147$ & \multirow{5}{*}{$\begin{array}{l}x^{2}=4.902 \\
d f=3 \\
p=.179 \\
\text { Cramer's } \\
V=0.128\end{array}$} \\
\hline Strongly Trust & $\begin{array}{c}35 \\
23.3 \%\end{array}$ & $\begin{array}{c}43 \\
29.3 \%\end{array}$ & \\
\hline Somewhat Trust & $\begin{array}{c}87 \\
58.0 \%\end{array}$ & $\begin{array}{c}87 \\
59.2 \%\end{array}$ & \\
\hline Somewhat Distrust & $\begin{array}{c}24 \\
16.0 \%\end{array}$ & $\begin{array}{c}12 \\
8.2 \%\end{array}$ & \\
\hline Strongly Distrust & $\begin{array}{c}4 \\
2.7 \%\end{array}$ & $\begin{array}{c}5 \\
3.4 \%\end{array}$ & \\
\hline Climbing Related Organizations & $\mathrm{N}=152$ & $\mathrm{~N}=149$ & \multirow{5}{*}{$\begin{array}{l}x^{2}=25.687 \\
d f=3 \\
p=.000 \\
\text { Cramer's } \\
V=0.292\end{array}$} \\
\hline Strongly Trust & $\begin{array}{c}63 \\
41.4 \%\end{array}$ & $\begin{array}{c}102 \\
68.5 \%\end{array}$ & \\
\hline Somewhat Trust & $\begin{array}{c}82 \\
53.9 \%\end{array}$ & $\begin{array}{c}47 \\
31.5 \%\end{array}$ & \\
\hline Somewhat Distrust & $\begin{array}{c}6 \\
3.9 \%\end{array}$ & $\begin{array}{c}0 \\
0.0 \%\end{array}$ & \\
\hline Strongly Distrust & $\begin{array}{c}1 \\
0.7 \%\end{array}$ & $\begin{array}{c}0 \\
0.0 \%\end{array}$ & \\
\hline Scientists in general & $\mathrm{N}=150$ & $\mathrm{~N}=148$ & \multirow{5}{*}{$\begin{array}{l}x^{2}=21.985 \\
d f=3 \\
p=.000 \\
\text { Cramer's } \\
V=0.272\end{array}$} \\
\hline Strongly Trust & $\begin{array}{c}66 \\
44.0 \%\end{array}$ & $\begin{array}{c}32 \\
21.6 \%\end{array}$ & \\
\hline Somewhat Trust & $\begin{array}{c}71 \\
47.3 \%\end{array}$ & $\begin{array}{c}82 \\
55.4 \%\end{array}$ & \\
\hline Somewhat Distrust & $\begin{array}{c}11 \\
7.3 \%\end{array}$ & $\begin{array}{c}28 \\
18.9 \%\end{array}$ & \\
\hline Strongly Distrust & $\begin{array}{c}2 \\
1.3 \%\end{array}$ & $\begin{array}{c}6 \\
4.1 \%\end{array}$ & \\
\hline NPS Rangers in general & $\mathrm{N}=151$ & $\mathrm{~N}=146$ & \multirow{5}{*}{$\begin{array}{l}x^{2}=59.301 \\
d f=3 \\
p=.000 \\
\text { Cramer's } \\
V=0.447\end{array}$} \\
\hline Strongly Trust & $\begin{array}{c}87 \\
57.6 \%\end{array}$ & $\begin{array}{c}23 \\
15.8 \%\end{array}$ & \\
\hline Somewhat Trust & $\begin{array}{c}57 \\
37.7 \%\end{array}$ & $\begin{array}{c}95 \\
65.1 \%\end{array}$ & \\
\hline Somewhat Distrust & $\begin{array}{c}6 \\
4.0 \%\end{array}$ & $\begin{array}{c}23 \\
15.8 \%\end{array}$ & \\
\hline Strongly Distrust & $\begin{array}{c}1 \\
0.7 \%\end{array}$ & $\begin{array}{c}5 \\
3.4 \%\end{array}$ & \\
\hline NPS Rangers at NERI & $\mathrm{N}=150$ & $\mathrm{~N}=148$ & \multirow{2}{*}{$x^{2}=51.977$} \\
\hline Strongly Trust & 88 & 28 & \\
\hline
\end{tabular}




\begin{tabular}{|l|c|c|l|}
\hline & $58.7 \%$ & $18.9 \%$ & $\mathrm{df}=3$ \\
\hline Somewhat Trust & 56 & 97 & $\mathrm{p}=.000$ \\
\hline Somewhat Distrust & $37.3 \%$ & $65.5 \%$ & Cramer's \\
& 5 & 19 & $\mathrm{~V}=0.418$ \\
\hline Strongly Distrust & $3.3 \%$ & $12.8 \%$ & \\
& 1 & 4 & \\
\hline Mainstream News Media & $0.7 \%$ & $2.7 \%$ & \\
\hline Strongly Trust & $\mathrm{N}=151$ & $\mathrm{~N}=145$ & $x^{2}=22.831$ \\
& 8 & 0 & $\mathrm{df}=3$ \\
\hline Somewhat Trust & $5.3 \%$ & $0.0 \%$ & $\mathrm{p}=.000$ \\
\hline Somewhat Distrust & 40 & 20 & $\mathrm{Cramer}$ 's \\
& $26.5 \%$ & $13.8 \%$ & $\mathrm{~V}=0.278$ \\
\hline Strongly Distrust & 68 & 62 & \\
\hline
\end{tabular}

Chapter 5: Discussion

The following chapter presents a discussion of the results found during statistical analysis. This discussion will further examine the findings and compare them to previous findings in outdoor recreation research. This chapter also includes implications and recommendations relevant to NERI managers.

\section{RQ1: Who are primary users of the cliffs in NERI?}

The purposeful selection of sampling sites appears to have yielded the hoped for split between two main trail user groups - hikers and climbers - as results found that $51 \%$ of respondents reported hiking as their primary activity, while $49 \%$ reported climbing.

While the overall sample was approximately $59 \%$ male and $41 \%$ female, hikers were almost exactly evenly split by gender. Climbers were noticeably different, with males making up $68 \%$ of the sample. This is consistent with Attarian's (1999) findings in the New River Gorge. Overall, survey respondents were highly educated, with $40 \%$ holding a four year college degree 
and $31 \%$ holding a masters, doctorate, or professional degree. However, $39 \%$ of hikers $(\mathrm{N}=61)$ held a masters, doctorate, or professional degree while only $22 \%(\mathrm{~N}=33)$ of climbers did. Again, these findings are consistent with Attarian's (1999) results. Survey respondents were also fairly young, with $47 \%$ of the overall sample in the $21-30$ age bracket. Fourteen percent of climbers were over the age of 40 . However, only $7 \%$ of climbers were over the age of 40 in Attarian's (1999) study. This is the only major difference in demographics between this study and previous studies (Attarian, 1999; Ramthun 2001). It is interesting that the number of climbers over the age of 40 has nearly doubled in the past 24 years, and perhaps points to a trend of more climbers choosing to continue climbing as they get older. This could indicate aging of the general climbing population, or could even be sampling bias related to differences in survey methodologies. Alternately, climbers over the age of 40 may be introduced to the sport through friends or climbing gyms. Overall, hikers (and to a lesser degree, climbers) were demographically consistent with other NPS site demographics (Attarian, 1999; Ramthun, 2001).

\section{RQ2: Do climbers and hikers differ in their attitudes toward cliff resource management?}

There were numerous significant differences between hikers and climbers in regards to their low impact behaviors and attitudes toward cliff resources management. In some regards, hikers appeared to be more observant of low impact behaviors, with the exception of practicing Leave No Trace. Climbers were more sensitive to large groups of climbers impacting their experience, but were also more resistant to the management of climbing areas, similar to previous findings by Attarian (1999) and Monz (2009).

The observed differences related to low impact behaviors between hikers and climbers in NERI are striking. Climbers agreed more strongly with the statement "I practice Leave No Trace when I hike/climb", but their mean scores for other low impact behavior statements did not 
reflect that. The low impact behavior statements that had the strongest statistically different results between hikers and climbers were: “The park provides clear indications about which trails should not be used" (H mean=3.93, C mean=3.40), "I feel a responsibility to help protect the plants and ecosystem in the park"( $\mathrm{H}$ mean=4.59, $\mathrm{C}$ mean=4.33), and "The continued use of unofficial visitor created trails is a serious threat to plants and soils along the park trails” (H mean $=4.09, \mathrm{C}$ mean=3.70). While their mean scores indicated that they were mostly in agreement with these statements, climbers as a group had a lower level of agreement with these statements than hikers. These lower mean score appears to indicate that there is a potential disconnect among climbers between how they perceive their low impact behavior and their actual behavior when climbing in NERI. These findings are consistent with Attarian's (1999) findings that more education may be necessary in order to prevent climbing impacts to cliff resources.

Climbers and hikers also differed in their preferences for cliff resource management. Of the 18 management statements that hikers and climbers were asked to respond to, the two groups differed significantly on 13 . Overall, climbers are less trustful of park managers, a theme that appears repeatedly throughout the results. They had a lower mean score than hikers on all of the questions relating to trust of park managers and belief that managers share their goals and values. Interestingly, climbers were more likely to feel that large groups of climbers detracted from their experience, but felt less strongly than hikers that that climbing group sizes should be limited. Additionally, climbers had a lower mean score on the statement "Climbing areas need an official management plan in order to be sustainable" and felt more strongly that managers did not have adequate knowledge of climbing to properly manage it at NERI. Monz (2009) also found participants in his study were more likely to be neutral or opposed to management of climbing 
areas, including the management of fixed bolts and anchors. Attarian (1999) reported similar results. He found that climbers positively agreed with the majority of the 25 behaviors and practices they were asked about (including the placement of top anchors), but were neutral on bolting.

Among climbers, the lack of trust in managers indicates that they will likely be more resistant to changes in climbing management policies in NERI (Monz, 2009). Managers will need to work with the climbing community to build trust and be seen as knowledgeable about the resource before deciding to make any changes to climbing policies in NERI if they want these changes to be successful (Schuster et al., 2001).

\section{RQ3: Do different sub-groups of climbers differ in their attitudes toward cliff resource management?}

In order to better understand the varying attitudes toward cliff resource management within the climbing community, climbers were also segmented based on their climbing style. By understanding the diverse opinions of the climbing community in NERI, managers will be better equipped to engage climbers in discussion, include them in management decisions, and attempt to reach a consensus through compromise. Additionally, identifying the source of problem behaviors can help managers create educational materials to address and correct misperceptions or illegal behavior (Borrie \& Harding, 2002).

Monz (2009) and Schuster et al. (2001) both segmented climbers by climbing style in order to identify differences in low impact behavior and attitudes toward climbing management within the greater climbing community. Similarly, this thesis attempted to identify differences in low impact behavior and attitudes toward climbing management within the climbing community 
in NERI. While some differences were found among the 5 sub-groups of climbers that were surveyed, there were not as many differences as expected based on earlier studies.

Only two differences occurred in the low impact behavior section. Trad climbers (mean=4.72) had a higher mean for the statement "I always use official trails" than top ropers (mean=4.08) and gym climbers (4.21). It is worth noting that all of the means across all of the groups were above 4 on a scale of 5 for this statement. However, in a previous survey question, climbers in general had a lower mean score than hikers for the statement "The park provides clear indications about which trails should not be used", indicating that climbers in general are unsure about which trails are official and which trails are not. This uncertainty related to official trails may make it easy for respondents to over or underestimate their use of official trails, and indicates that the park must do a better job of labeling official trails and closing unofficial ones, especially in climbing areas. In addition to significant differences among climbers on the use of official trails, there were also significant differences on the issue of chiseling holds. All groups differed significantly from each other as to whether it is appropriate to chisel holds. While all groups disagreed that it is an acceptable practice, top ropers $(\mathrm{M}=1.82)$ and gym climbers $(\mathrm{M}=1.86)$ displayed higher levels of agreement than sport $(\mathrm{M}=1.31)$, $\operatorname{trad}(\mathrm{M}=1.13)$, and hybrid climbers $(\mathrm{M}=1.04)$.

Schuster et al. (2001) in particular found several differences among climbers when they were segmented by climbing style. The authors found that climbers' self-perception indicated that traditional climbers thought that climbers in general did not adequately practice land stewardship and had negative attitudes toward management in general. Similarly, this study found that trad climbers were significantly less trustful of managers and management goals. 
Monz (2009) also segmented climbers by climbing type. However, he surveyed climbers on environmental impacts rather than low impact behavior, and thus direct comparisons between Monz (2009) and this thesis are difficult. The only significant difference that occurred based on climbing style was in regards to damage to trees, which sport climbers found more offensive than traditional climbers.

Two themes appear in this research question and again in the following questions. There are significant, practical differences within the climbing community in regards to knowledge of climbing regulations. Additionally, there are significant, practical differences within the climbing community in regards to trust of NERI managers. Trad climbers tend to have greater knowledge of low impact behavior and tend to have a higher level of agreement that they engage in low impact behaviors. However, they are less trustful of managers overall. Similarly, more experienced climbers also tend to have greater knowledge and higher compliance with low impact behaviors, but they are also less trustful of managers. Previous studies have established that climbers are a diverse group (Attarian, 1999; Borrie \& Harding, 2002; Monz, 2009; Ramthun, 2001; Schuster et al. 2001) and that education and collaboration are crucial to earning trust and gaining cooperation from climbers (Borrie \& Harding, 2002; Monz, 2009; Schuster et al., 2001). Due to the amount of diversity within the climbing community, it is important to understand where different groups stand on management issues and low impact behavior in order to better target educational and collaborative efforts. For example, providing advanced/expert climbers with educational materials designed for beginners may be insulting, while attempting to collaborate with trad climbers prior to establishing trust may further alienate them.

These results indicate that NERI managers should develop a succinct, standardized summary of climbing regulations in NERI in order to establish a clear, consistent baseline for 
low impact behavior. These regulations then need to be published in nontraditional (non-NPS) media in order to reach all members of the climbing community. Potential outlets for new information for climbers include guidebooks such as New River Gorge Rock Climbs (Williams, 2010), the New River Alliance of Climbers (NRAC) Facebook and web pages, and Waterstone

Outfitters. This is especially important in light of Smaldone \& McKenney's (2013) finding that show that less than $10 \%$ of climbers use NPS sources when looking for information while planning a trip to NERI.

RQ4: Do climbers of different climbing abilities differ in their attitudes toward cliff resource management?

Like climbing style, climbing ability appeared to have some impact on climber's low impact behaviors and attitudes towards cliff resource management. There were significant differences in six of the low impact behavior statements and three of the cliff resource management statements. Four of the nine significant statements had to do with the use of permanent bolts and anchors, an issue which has a contentious history in NERI and continues to be a source of conflict between climbers and managers. Two of the significant statements had to do with the use of official trails. The use of unofficial trails is also of concern to park managers.

Intermediate climbers $(\mathrm{M}=4.74)$ reported higher levels of agreement with the statement “Permanent anchors provide easy access to routes" while novice climbers (M=2.64) reported higher levels of agreement with the statement "Permanent anchors are not needed". Advanced/expert $(\mathrm{M}=4.71)$ and intermediate climbers $(\mathrm{M}=4.71)$ reported higher levels of agreement with the statement "Fixed anchors should be used to help preserve the environment". Advanced/expert climbers $(\mathrm{M}=4.18)$ reported higher levels of agreement with the statement "The impact of bolts and anchors on the natural resource is minimal”. Advanced/expert climbers also 
reported the highest levels of agreement with the statements "I always use official trails" $(\mathrm{M}=4.53)$ and "I avoid trails that have been closed" $(\mathrm{M}=4.71)$.

The remaining significant statements were related to knowledge and trust. Novice climbers reported higher levels of agreement with the statement "Chiseling holds is an acceptable practice" $(\mathrm{M}=1.91)$ and lower levels of agreement with the statement "I am familiar with the climbing regulations at the $N R G$ " $(\mathrm{M}=2.69)$. This statement was perhaps the most practically significant, as intermediate climbers $(\mathrm{M}=3.59)$ and advanced/expert climbers $(\mathrm{M}=4.17)$ displayed positive agreement with this statement while novice climbers did not. Finally, advanced/expert climbers $(M=3.16)$ reported lower levels of agreement with the statement "I trust that the NPS managers at the NRG will do what is right for the park".

The greatest differences in low impact behaviors within the NERI climbing community were found when climbers were segmented by ability. In general, more experienced climbers were more familiar with climbing regulations, used official trails and avoided closed trails more frequently, were more in favor of using fixed anchors to help preserve the environment, and felt that NPS managers at NERI did not have the same goals as them. Borrie and Harding (2002) segmented climbers based on experience as well. They found that climbers with less experience climbing were significantly less comfortable with the idea of placing a new bolted route. Novice climbers in this thesis expressed more positive agreement with statements related to bolting. However, they were not asked if they would be comfortable placing a route themselves.

Several similarities were consistent with previous research questions. Climbers differed on their familiarity with climbing regulations, as well as their trust of park manager's actions. Additionally, climbers differed on their attitudes toward the use of permanent bolts and anchors and their use of official trails. NERI managers should draft a summary of the official bolt and 
anchor regulations (with a brief explanation of why such regulations are in place) and as noted previously, disseminate it through nontraditional (non-NPS) channels such as New River Gorge Rock Climbs (Williams, 2010), the New River Alliance of Climbers (NRAC) Facebook and web pages, and Waterstone Outfitters. Additionally, managers should close and restore (revegetate as possible, etc.) unofficial trails (or make them official) and provide updated maps and signage of official trails to all park visitors.

\section{RQ5: Does where (indoors vs. outdoors) climbers learn to climb influence their attitudes}

\section{toward cliff resource management?}

Unlike climbing style and climbing ability, where a climber learns to climb did not appear to have a significant impact on their attitudes toward low impact behaviors or cliff resource management. In the NERI sample, there were only two statistically significant differences between the two groups, one in regards to low impact behavior, and one in regards to cliff resource management. Climbers who learned to climb indoors had higher agreement with the statement "Permanent bolted anchors should be used at the top of climbs" than climbers who learned to climb outdoors. Climbers who learned to climb both indoors and outdoors (evenly split between the two) did not differ significantly from either group. Climbers who learned to climb indoors showed lower agreement with the statement "I am familiar with the climbing regulations at the NRG" than climbers who learned outdoors. Again, climbers who learned to climb both indoors and outdoors did not differ significantly from the other two groups.

Borrie and Harding (2002) compared climbers based on their mode of introduction to rock climbing and their level of experience with climbing (in particular at their research site), and determined that these two variables have a significant impact on a climbers' attitudes toward low impact behavior. While their study was severely limited by weather which limited their 
sampling and hence ability to generalize, they did find that climbers who learned to climb indoors had significantly higher reservations about climbing near Native American artifacts and placing bolts on routes. However, this thesis indicates that climbers who learned to climb indoors are significantly more in favor of placing permanent bolts at the top of climbs. It should be noted, however, that they were not asked about their comfort level with placing the bolts themselves.

It is notable that there appear to be very few differences in low impact behaviors and attitudes toward management based on where a climber learns to climb. With the increased prevalence of climbing gyms, more and more climbers have their first climbing experience and many of their formative climbing experiences indoors (Rapelje, 2004). If learning to climb indoors had contributed to differences within the climbing community, especially misperceptions about climbing rules or regulations, managers at NERI may have needed to address those through educational materials targeted at indoor climbers. Future studies should continue to address this issue.

\section{RQ6: Does how (formally trained vs. informally) climbers learn to climb influence their attitudes toward cliff resource management?}

The method by which climbers learn to climb appeared to have some influence on their low impact behaviors and attitudes toward cliff resource management, largely with regards to climber's knowledge and education. Climbers who learned to climb formally differed significantly from climbers who learned to climb informally on four statements. Two statements were low impact behavior statements, and two were cliff resource management statements.

Climbers who learned to climb formally reported higher levels of agreement with the statements "I try to keep my noise level down", and "All climbing guide services should be required to be 
certified or accredited". Climbers who learned to climb formally also reported higher levels of agreement with the statement "Climbers need to be educated concerning their role in the management process". Climbers who learned to climb informally reported higher levels of agreement with the statement "Soils and plants along the trails are hardy and not easily damaged".

Climbers who learned to climb formally appear to be more concerned with the qualifications of climbing guide services, climbing etiquette and climber's knowledge about the resource. This may be a result of learning how to climb in a more formal setting, with experienced, certified climbers who take the time to emphasize the importance of such qualities. NERI managers should consider developing climbing specific interpretive materials that emphasize important facts about cliff resources, and explain why they ban practices such as chiseling holds or unpermitted bolting. Interpretive materials such as a guide to common plant species found on NERI cliffs can help educate climbers about the unique cliff resources in NERI and build trust with the climbing community (Attarian, 1999; Monz, 2009). Any interpretive material would need to be disseminated through non-NPS sources. Specifically, interpretive materials should be included in New River Gorge Rock Climbs by Mike Williams, as well as on the NRAC Facebook page and official website. Information intended for novices should be placed prominently in the guidebook, while information for expert/advanced climbers might be better disseminated through NRAC and Waterstone, the local outfitters. The specific channels to use to communicate with different levels of climbers based on their climbing ability could also be addressed in future research.

\section{RQ7: Do hikers and climbers differ in who they trust for information on cliff resources?}


Hikers and climbers displayed strongly significant differences in regards to who they trusted for information on cliff resources. Notably, climbers displayed stronger trust of climbing organizations and weaker trust of environmental organizations, scientists, and NPS rangers in general and at NERI. Attarian (1999), Monz (2009), Schuster et al. (2001), and Borrie and Harding (2002) have all found climbers to be less trustful of managers as well. Previous research indicates that low levels of trust also indicate lower levels of compliance with regulations (Winter \& Knap, 2001). Additionally, higher levels of trust have been found to be beneficial for facilitating conservation based behaviors and civic involvement (Payton et al., 2005; Winter \& Cvetkovich, 2010).

In addition being less trustful of NPS rangers, both at NERI and in general, climbers also turn to non-NPS sources for information about climbing and cliff resource management (Smaldone \& McKenney, 2013). Any climbing information provided on the NPS website or at the visitor centers for NERI often goes overlooked by climbers. Climbers' lack of trust combined with their tendency to seek climbing and cliff resource information from non-NPS sources indicates that NERI managers are missing their key audience for climbing and cliff resource management. In order for NERI managers to reach climbers, they need to develop clear, consistent messages communicating behavioral expectations, as well as any potential future cliff management actions. These messages need to be dispersed through non-NPS sources, such as climbing websites, magazines, newsletters, NRAC, Waterstone Outfitters, and local climbing guidebooks.

Future research in NERI should continue to follow climbers and the knowledge gaps and behavioral differences that exist between the different sub-groups of climbers. Additionally, future research should monitor climbers' trust of managers as well as actions taken by managers 
to reach out to climbers and increase their levels of trust. Managers should also continue to monitor where climbers turn for information in order to ensure that they are receiving accurate, up-to-date information on climbing in NERI, as well as to address any misperceptions that may be spread by inaccurate information.

\section{Conclusions}

Overall, NERI climbers and hikers were found to be a distinct yet diverse user groups. Climbers were found to be less trusting of park managers and management actions, more sensitive to the presence of large groups of climbers, and more knowledgeable about low impact behaviors when compared to hikers. However, some interesting patterns emerged when climbers were segmented by climbing style, experience level, learning environment, and learning style and compared with each other.

Trad climbers exhibited higher levels of knowledge and lower levels of trust, compared to gym climbers and, in some cases, top ropers. Compared to previous research (Schuster et al., 2001), fewer than expected differences occurred. However, when climbers were segmented by experience level, more differences than expected occurred when compared with previous research (Borrie \& Harding, 2002; Monz 2009). Not surprisingly, more experienced climbers were more knowledgeable about low impact behaviors and engaged in them more frequently. Novice climbers did not see as much of a need for permanent bolts and anchors compared to intermediate and advanced/expert climbers, indicating an opportunity for NERI managers to develop educational material targeting this group and explaining why bolts and anchors are placed and how they should be used.

Fewer than expected differences also occurred when climbers were segmented by learning environment (indoors vs. outdoors). Only two statements (one management and one low 
impact behavior) were significant. This contradicts many of the anecdotal concerns that experienced climber, guides, and some managers expressed about climbers who learned to climb indoors having less knowledge and practice of low impact behaviors and climbing regulations.

Little research has been done on rock climbers segmented by learning style (formal vs. informal). This thesis found a few interesting differences between the two groups. Climbers who learned to climb in a formal environment were more knowledgeable about climbing regulations and were more concerned with climbers' etiquette and behavior and the qualifications of climbing guides and services.

The findings of this thesis, combined with the findings of Smaldone and McKenney (2013), indicate that NERI managers need to focus on increasing their credibility and trust in the eyes of rock climbers. They should start by making their values and management goals clear to both user groups, considering the number of respondents who either did not respond, or responded "don't know" to questions relating to managers' goals, values, and qualifications. Additionally, this thesis found some key differences in low impact practices and knowledge of park resources both between hikers and climbers and within the climbing community at NERI. NERI managers need to develop educational materials that address these knowledge gaps and to encourage the types of low impact behaviors they would like to see implemented at hiking and climbing sites near the cliffs. This material should be dispersed to the specific appropriate target audiences, through channels like climbing guide books (New River Gorge Rock Climbs), NRAC's website, and local climbing businesses such as Waterstone Outfitters in order to reach climbers, who rarely use NPS information sources. 


\section{References}

Anheier, H. \& Kendall, J. (2002). Interpersonal trust and voluntary associations: Examining three approaches. British Journal of Sociology, 53, (3), 343-362.

Ansari, A. M. (2008). Understanding the motivations of rock climbers: A social worlds study. (Master's Thesis, University of Nevada Las Vegas)

Attarian, A. (1999). Factors influencing responsible rock climbing behavior. Unpublished manuscript, The American Alpine Club, Golden, CO. 28 pp.

Borrie, W.T., \& Harding, J.A. (2002). Effective recreation visitor communication strategies: Rock climbers in the Bitterroot Valley, Montana. USDA Forest Service Research Note RMRS-RN-15.

Brehm, J., \& Rahn, W. (1997). Individual-level evidence for the causes and consequences of social capital. American Journal of Political Science, 41, 999-1023.

Bryan, H. (1979). Conflict in the great outdoors: Toward understanding and managing for diverse sportsmen preferences (No. 4). Alabama: Bureau of Public Administration, University of Alabama.

Bryan, H. (2000). Recreation specialization revisited. Journal of Leisure Research, 32(1).

Cvetkovich, G.T. \& Lofstedt, R.E. (Eds.). (1999). Social trust and the management of risk. London: Earthscan Publications Ltd.

Cvetkovich, G.T. \& Winter, P.L. (1998). Community reactions to water quality problems in the Colville National Forest: Final report. Submitted to the Colville National Forest, Washington, and the San Dimas Technology and Development Center, San Dimas, CA. $32 \mathrm{p}$.

Farrell, T.A., Hall, T.E., \& White, D.D. (2001). Wilderness campers' perception and evaluation of campsite impacts. Journal of Leisure Research, 33, 229-250.

Flora, C.B. (2000). Measuring the social dimensions of managing natural resources. In Fulton, D.C., Nelson, K.C., Anderson, D.H., \& Lime, D.W. (eds.) Human dimensions of natural resource management: Emerging issues and practical applications (pp. 83-99). St. Paul: Cooperative Park Studies Program, University of Minnesota, Department of Forestry Resources. 
Frentz, I.C., Voth, D.E., Burns, S., \& Sperry, C.W. (2000). Forest service-community relationship building: Recommendations. Society of Natural Resources, 13, 549-566.

Hendee, Stankey, \& Lucas, 1990. Wilderness management. 2nd ed. Golden, CO: North American Press. 568 p.

Manfredo, M. J., Yuan, S. M., \& McGuire, F.A. (1992). The influence of attitude accessibility on attitude-behavior relationships: Implications for recreation research. Journal Of Leisure Research, 24(2), 157.

Manning, R. (1999). Studies in Outdoor Recreation. Corvallis, OR: Oregon State University Press.

Miller, Borrie, \& Harding, 2001. Basic knowledge of factors that limit the practice of low-impact behaviors. Unpublished report on file at: U.S. Department of Agriculture, Forest Service, Rocky Mountain Research Station, Aldo Leopold Wilderness Research Institute, Missoula, MT. 182 p. http://www.wilderness.net/research.cfm\#library.

Monz, C.A. (2009). Climbers' attitudes toward recreation resource impacts in the Adirondack Park's Giant Mountain Wilderness. International Journal of Wilderness, 15, 26-33.

Moser, S. \& Davidson, S. (1999). Technical rock climbing trends in the United States. In Cordell, H.K. (PI) Outdoor recreation in American live: A national assessment of demands and supply trends. Champaign: Sagamore Publishing,

Outdoor Industry Foundation. (2010). Outdoor recreation participation report: A look at trends in American participation in outdoor recreation with a focus on youth, diversity, and the future of the outdoors. Retrieved April 28, 2013, from the Outdoor Industry Foundation. Web site: http://www.outdoorindustryfoundation.org.

Payton, M.A., Fulton, D.C., Anderson, D.H. (2005). Influence of place attachment and trust on civic action: A study at Sherburne National Wildlife Refuge. Society and Natural Resources, 18, 511-528. doi: 10.1080/08941920590947940

Ramthun, R. \& Blake, A. (2002). Exploring quality standards for New River Gorge climbing sites: Establishing a baseline for the future. In Proceedings of the 2002 Northeastern Recreation Research Symposium, 2002 April 13-16, (pp. 87-89). Bolton Landing, NY.

Rapelje, B. W. (2004). Rock climbing sub-worlds: a segmentation study (Doctoral dissertation, Texas A\&M University).

Schuster, R.M., Thompson, J.G., \& Hammit, W.E. (2001). Rock climbers' attitudes toward management of climbing and the use of bolts. Environmental Management, 28 (3), 403-412. DOI: $10.1007 / \mathrm{s} 002670010232$ 
Siegrist, M., Cvetkovich, G.T., \& Roth, C. (2000). Salient value similarity, social trust, and risk/benefit perception. Risk Analysis, 20(3), 353-362.

Smaldone, D., \& McKenney, K. (2013). Recreation in the New River Gorge National River: An evaluation of the activities and attitudes of hikers and climbers toward cliff resource management. National Park Service Technical Report

Stankey, G.H. (1972). A strategy for the definition and management of wilderness quality. In J.V. Krutilla (ed.), Natural environments, studies in theoretical and applied analysis. Baltimore: The Johns Hopkins University Press

Thompson, K. A. (2010). Social Worlds of Rock Climbers at Seneca Rocks, West Virginia (Doctoral dissertation, West Virginia University Libraries).

Tourangeau, R, Rips, L.J., \& Rasinski, K. (2000). The psychology of survey response. New York: Cambridge University Press. 401 p.

VanVoorhis, C. R. W., \& Morgan, B. L. (2007). Understanding power and rules of thumb for determining sample sizes. Tutorials in Quantitative Methods for Psychology, 3(2), 43-50.

White, D.D., Hall, T.E., \& Farrell, T.A. (2001). Influence of ecological impacts and other campsite characteristics on wilderness visitors' campsite choices. Journal of Park and Recreation Administration, 19, 83-87.

Williams, Mike. (2010). New River Gorge Rock Climbs. Wolverine Publishing.

Winter, P.L. \& Cvetkovich, G.T. (2010). Trust mediates conservation-related behaviors. Ecopsychology, 2(4), 211-219. DOI: 10.1089/eco.2010.0046

Winter, P.L. \& Knap, N. (2001). An exploration of recreation and management preferences related to threatened and endangered species: Final report for the Angeles, Cleveland, Los Padres, and San Bernadino National Forests. Unpublished report, submitted to the SCCS core team and the four southern California National Forests.

Wynveen, Christopher J.; Kyle, Gerard T.; Theodori, Gene L. 2009. The relationship between place bonding and social trust, as explored in a study in the Big Thicket National Preserve, Texas. In: Klenosky, David B.; Fisher, Cherie LeBlanc, eds. Proceedings of the 2008 Northeastern Recreation Research Symposium; Bolton Landing, NY. 


\title{
New River Gorge National River
}

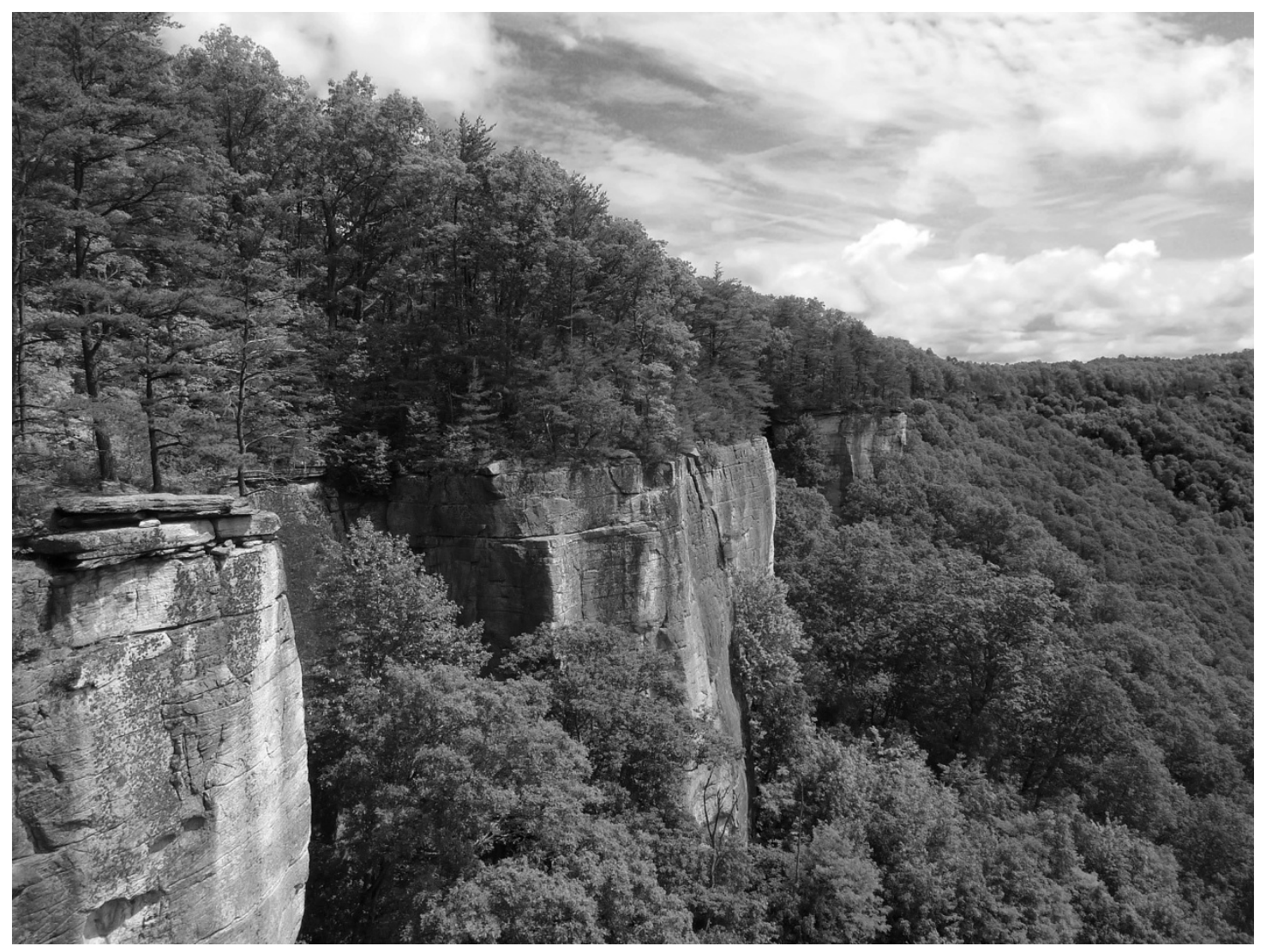

\section{A survey of visitors: Your opinion matters!}

\author{
Conducted by: \\ Dr. Dave Smaldone \\ Recreation, Parks \& Tourism Program \\ Division of Forestry \& Natural Resources \\ West Virginia University \\ In cooperation with the New River Gorge National River
}




\section{Survey Directions:}

Thank you for taking the time to complete this survey. The results of this study will help resource managers better understand visitors like you, as well as develop education and interpretive programs for park visitors. This survey is only being given to a select number of visitors, so your participation is very important. Your participation is voluntary, and your responses will be kept confidential and anonymous. This survey should take about 15 minutes of your time. Please read the instructions for each question and answer to the best of your ability.

First, we would like to find out a little about your current visit to the New River Gorge National River.

1. Is this your first trip to the New River Gorge (NRG)? (please check one) $\mathrm{N}=160$

$\square$ Yes $40 \% \quad \square$ No $60 \%$

2. How long is your current trip to the NRG? (please check one, and write in number) $\mathrm{N}=140$

$\square$ Less than one day. $35 \% \rightarrow$ If so, how many hours?

More than a day. $65 \% \rightarrow$ If so, how many days?

3. What activities are you doing today while on the trail? (check all that apply) $\mathrm{N}=160$

$\square$ hiking/walking $94.4 \% \quad \square$ rock climbing $5 \%$

$\square$ bird watching $14.4 \%$

$\square$ photography $48.1 \%$

$\square$ viewing wildlife (other than birds) $23.1 \% \square$ viewing the scenery $72.5 \%$

$\square$ visiting historic sites $11.9 \%$

other: $20 \%$

$\rightarrow$ Now, from the activities that you checked above, please write in the one that was most important to you: $\mathrm{N}=138$

hiking/walking $47.8 \% \quad \square$ rock climbing $2.9 \%$

$\square$ bird watching $1.4 \% \quad \square$ photography $2.2 \%$

viewing wildlife (other than birds) $0.7 \% \square$ viewing the scenery $27.5 \%$

visiting historic sites $0.7 \% \quad \square$ picnicking $0 \%$

other: $16.7 \%$

4. How did the number of people you saw during your visit compare with what you expected?

$\mathrm{N}=160$

A lot less than what you expected $15 \%$

A little less than what you expected $20.6 \%$

About what you expected $48.1 \%$

$\square$ A little more that what you expected $10.6 \%$

A lot more than what you expected 3.8\%

You did not have any expectations $1.9 \%$ 
5. How crowded did you feel on your visit to the New River Gorge today? (circle one number) $\mathrm{N}=160$

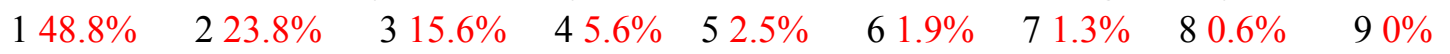

Not crowded at all

Slightly Crowded

Moderately Crowded

Extremely Crowded

5. How important to you are the following features at NRG hiking areas? (please circle one response for each question)

\begin{tabular}{|c|c|c|c|c|c|c|}
\hline \multirow[b]{2}{*}{ Restrooms at trailheads... } & \multicolumn{3}{|c|}{ Not important } & \multicolumn{3}{|c|}{ Very Important } \\
\hline & 2 & 3 & 4 & 5 & 6 & $7 \mathrm{~N}=158$, Mean=4.34 \\
\hline Parking Areas........... & 2 & 3 & 4 & 5 & 6 & $7 \mathrm{~N}=159$, Mean $=5.61$ \\
\hline Signage at Trail Access Points ...............1 & 2 & 3 & 4 & 5 & 6 & $7 \mathrm{~N}=159$, Mean $=5.82$ \\
\hline Trail Quality.... & 2 & 3 & 4 & 5 & 6 & $7 \mathrm{~N}=159$, Mean $=5.41$ \\
\hline Visitor Center... & 2 & 3 & 4 & 5 & 6 & $7 \mathrm{~N}=158$, Mean $=3.93$ \\
\hline Ranger presence & 2 & 3 & 4 & 5 & 6 & $7 \mathrm{~N}=160$, Mean=2.97 \\
\hline Suitable campsites in park. & 2 & 3 & 4 & 5 & 6 & $7 \mathrm{~N}=159$, Mean $=4$ \\
\hline
\end{tabular}

6. How would you rate your satisfaction with the quality of these features at NRG hiking areas? (circle one response for each question)

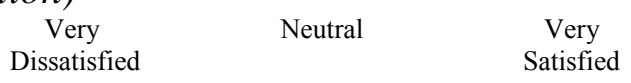

\begin{tabular}{|c|c|c|c|c|c|c|}
\hline Restrooms at trailheads. & 2 & 3 & 4 & 5 & 6 & $7 \mathrm{~N}=152$, Mean $=4.88$ \\
\hline Parking Areas............ & 2 & 3 & 4 & 5 & 6 & $7 \mathrm{~N}=158$, Mean $=5.84$ \\
\hline Signage at Trail Access Points.. & 2 & 3 & 4 & 5 & 6 & $7 \mathrm{~N}=158$, Mean $=5.73$ \\
\hline Trail Quality. & 2 & 3 & 4 & 5 & 6 & $7 \mathrm{~N}=158$, Mean $=6.01$ \\
\hline Visitor Center.... & 2 & 3 & 4 & 5 & 6 & $7 \mathrm{~N}=152$, Mean $=5.63$ \\
\hline Ranger presence on trails. & 2 & 3 & 4 & 5 & 6 & $7 \mathrm{~N}=149$, Mean $=4.45$ \\
\hline Suitable campsites in park. . & 2 & 3 & 4 & 5 & 6 & $7 \mathrm{~N}=143$, Mean=4.39 \\
\hline
\end{tabular}

Now, we'd like you to tell us about your background: (circle or fill in as appropriate):

8. How many years have you been hiking? (please write in number)

9. How many years have you been hiking at the New River Gorge? (write in number)

10. When was your first visit to New River Gorge? (write in the month and year) Month: _ Y Year: _ $\square$ Don't know/can't remember

11. Approximately how many times a year do you participate in hiking? (check one) $\mathrm{N}=161$
$\square<10$ days/year $29.2 \%$
$\square 31-40$ days/year $7.5 \%$
$\square$ 11-20 days/year $24.2 \%$
41-50 days/year $2.5 \%$
$\square$ 21-30 days/year $16.1 \%$
$>50$ days/year $20.5 \%$ 
12. Approximately how many times a year do you participate in hiking at the New River Gorge? (check one ) $\mathrm{N}=159$
$\square<10$ days/year $79.2 \%$
$31-40$ days/year $3.8 \%$
$\square$ 11-20 days/year 3.1\%
41-50 days/year $1.9 \%$
21-30 days/year $5.0 \%$
$>50$ days $/$ year $6.9 \%$

13. What category best describes your overall ability as a hiker? (check one ) $\mathrm{N}=161$

$\square$ Novice $12.4 \% \quad \square$ Intermediate $50.9 \% \quad \square$ Advanced $32.3 \%$

$\square$ Expert $4.3 \%$

14. From your home, how many hours did you travel to reach the New River Gorge? (please check one box) $\mathrm{N}=161$

$\square$ Less than $117.4 \% \square 1$ to $213.7 \% \square 3$ to $420.5 \% \square 5$ to $624.2 \% \square 7$ to $88.1 \%$ more than $816.1 \%$

15. What would you be most interested in learning about, in regards to the cliffs and their ecosystems at the NRG? (please write in your answer)

The following questions deal with the cliffs at the NRG and your opinion of their importance. Please place a check under the column that best describes how strongly you agree or disagree with each of the following statements. (check one box for each question)

\begin{tabular}{|l|c|c|c|c|c|c|}
\hline 16. & $\begin{array}{c}\text { Strongly } \\
\text { Disagree }\end{array}$ & Disagree & Neutral & Agree & $\begin{array}{c}\text { Strongly } \\
\text { agree }\end{array}$ & $\begin{array}{c}\text { Don't } \\
\text { know }\end{array}$ \\
\hline $\begin{array}{l}\text { a. The cliffs in the NRG are the same } \\
\text { as cliffs anywhere else. N=149, } \\
\text { Mean=2.03 (Total N=159) }\end{array}$ & $23.3 \%$ & $49.7 \%$ & $17 \%$ & $2.5 \%$ & $1.3 \%$ & $6.3 \%$ \\
\hline $\begin{array}{l}\text { b. } \text { Cliffs are a fundamental resource to } \\
\text { the NRG National River, and } \\
\text { essential to its national significance. } \\
\text { N=157, Mean=4.44 (Total N=159) }\end{array}$ & $2.5 \%$ & $0.6 \%$ & $8.2 \%$ & $27 \%$ & $60.4 \%$ & $1.3 \%$ \\
\hline $\begin{array}{l}\text { c. The cliffs in the NRG provide } \\
\text { habitat for rare plants. N=128, } \\
\text { Mean=4.08 (Total N=159) }\end{array}$ & $2.5 \%$ & $0.6 \%$ & $16.4 \%$ & $29.6 \%$ & $31.4 \%$ & $19.5 \%$ \\
\hline $\begin{array}{l}\text { d. The cliffs in the NRG provide } \\
\text { habitat for rare animals. N=126, } \\
\text { Mean=3.96 (Total N=158) }\end{array}$ & $1.9 \%$ & $1.3 \%$ & $22.2 \%$ & $27.2 \%$ & $27.2 \%$ & $20.3 \%$ \\
\hline $\begin{array}{l}\text { e. The cliffs in the NRG support } \\
\text { unique habitats and ecosystems. } \\
\text { N=143, Mean=4.23 (Total N=158) }\end{array}$ & $1.9 \%$ & $1.9 \%$ & $13.3 \%$ & $29.7 \%$ & $43.7 \%$ & $9.5 \%$ \\
\hline
\end{tabular}




\begin{tabular}{|l|l|l|l|l|l|l|}
\hline $\begin{array}{l}\text { f. The views of natural landscape } \\
\text { from the cliffs in the NRG are } \\
\text { worth protecting from human } \\
\text { development. N=142, Mean=4.68 } \\
\text { (Total N=158) }\end{array}$ & $3.2 \%$ & $0.6 \%$ & $3.8 \%$ & $6.3 \%$ & $75.9 \%$ & $10.1 \%$ \\
\hline $\begin{array}{l}\text { g. The views from the cliffs in the } \\
\text { NRG are one of the Park's most } \\
\text { important and unique resources. } \\
\mathrm{N}=143, \text { Mean=4.59 (Total N=158) }\end{array}$ & $2.5 \%$ & $0.6 \%$ & $5.1 \%$ & $15.2 \%$ & $67.1 \%$ & $9.5 \%$ \\
\hline
\end{tabular}

This next section has questions about your behaviors and opinions regarding hiking practices. Please place a check under the column that best describes how strongly you agree or disagree with each of the following statements. (check one box for each question)

\begin{tabular}{|l|c|c|c|c|c|c|}
\hline 17. & $\begin{array}{c}\text { Strongly } \\
\text { Disagree }\end{array}$ & Disagree & Neutral & Agree & $\begin{array}{c}\text { Strongly } \\
\text { agree }\end{array}$ & $\begin{array}{c}\text { Don't } \\
\text { know }\end{array}$ \\
\hline $\begin{array}{l}\text { a. I always pack out my trash. } \\
\text { N=160, Mean=4.89 (Total } \\
\mathrm{N}=161 \text { ) }\end{array}$ & $0.6 \%$ & $0.6 \%$ & $0 \%$ & $6.8 \%$ & $91.3 \%$ & $0.6 \%$ \\
\hline $\begin{array}{l}\text { b. I always use official trails. } \\
\mathrm{N}=160, \text { Mean=4.24 (Total } \\
\mathrm{N}=161)\end{array}$ & $0.6 \%$ & $7.5 \%$ & $9.9 \%$ & $31.1 \%$ & $50.3 \%$ & $0.6 \%$ \\
\hline $\begin{array}{l}\text { c. I respect revegetation efforts. } \\
\text { N=159, Mean=4.8 (Total N=161) }\end{array}$ & $0 \%$ & $0 \%$ & $0.6 \%$ & $18.6 \%$ & $79.5 \%$ & $1.2 \%$ \\
\hline $\begin{array}{l}\text { d. I avoid trails that have been } \\
\text { closed. N=159, Mean=4.51 (Total } \\
\mathrm{N}=161)\end{array}$ & $0.6 \%$ & $1.2 \%$ & $8.1 \%$ & $26.1 \%$ & $62.7 \%$ & $1.2 \%$ \\
\hline $\begin{array}{l}\text { e. I practice Leave No Trace when I } \\
\text { hike. N=130, Mean=4.59 (Total } \\
\mathrm{N}=153 \text { ) }\end{array}$ & $0 \%$ & $0.7 \%$ & $10.5 \%$ & $11.8 \%$ & $62.1 \%$ & $15 \%$ \\
\hline $\begin{array}{l}\text { f. I try to keep my noise level down. } \\
\text { N=160, Mean=4.39 (Total } \\
\mathrm{N}=161)\end{array}$ & $0 \%$ & $3.1 \%$ & $10.6 \%$ & $30.6 \%$ & $55.6 \%$ & $0.6 \%$ \\
\hline $\begin{array}{l}\text { g. I park my vehicle in designated } \\
\text { areas. N=159, Mean=4.72 (Total } \\
\text { N=160) }\end{array}$ & $0 \%$ & $0.6 \%$ & $1.3 \%$ & $23.8 \%$ & $73.8 \%$ & $0.6 \%$ \\
\hline $\begin{array}{l}\text { h. Soils \& plants along the Park } \\
\text { trails are hardy and not easily } \\
\text { damaged. N=150, Mean=2.55 } \\
\text { (Total N=160) }\end{array}$ & $23.8 \%$ & $34.4 \%$ & $11.9 \%$ & $8.1 \%$ & $15.6 \%$ & $6.3 \%$ \\
\hline $\begin{array}{l}\text { i. I respect seasonal closures at } \\
\text { certain trails for wildlife needs. } \\
\text { N=158, Mean=4.57 (Total }\end{array}$ & $0.6 \%$ & $1.3 \%$ & $6.3 \%$ & $23.9 \%$ & $67.3 \%$ & $0.6 \%$ \\
\hline
\end{tabular}




\begin{tabular}{|c|c|c|c|c|c|c|}
\hline $\mathrm{N}=159)$ & & & & & & \\
\hline $\begin{array}{l}\text { j. The park provides clear } \\
\text { indications about which trails } \\
\text { should not be used. } N=140 \text {, } \\
\text { Mean=3.94 (Total } N=159 \text { ) }\end{array}$ & $1.3 \%$ & $7.5 \%$ & $17.6 \%$ & $30.8 \%$ & $30.8 \%$ & $11.9 \%$ \\
\hline $\begin{array}{l}\text { k. Compared to other people I saw } \\
\text { on this visit, I am causing less } \\
\text { damage to the soils \& vegetation. } \\
\mathrm{N}=117 \text {, Mean }=3.49 \text { (Total } \\
\mathrm{N}=160 \text { ) }\end{array}$ & $2.5 \%$ & $1.3 \%$ & $42.5 \%$ & $11.9 \%$ & $15 \%$ & $26.9 \%$ \\
\hline $\begin{array}{l}\text { 1. I feel a responsibility to help } \\
\text { protect the plants and ecosystems } \\
\text { in the Park. } \mathrm{N}=161, \text { Mean }=4.59 \\
(\text { Total } \mathrm{N}=161)\end{array}$ & $1.9 \%$ & $0 \%$ & $4.3 \%$ & $24.8 \%$ & $68.9 \%$ & $0 \%$ \\
\hline $\begin{array}{l}\mathrm{m} \text {. The continued use of unofficial } \\
\text { visitor created trails is a serious } \\
\text { threat to plants and soils along the } \\
\text { Park trails. } \mathrm{N}=144 \text {, Mean }=4.09 \\
(\text { Total } \mathrm{N}=158)\end{array}$ & $3.2 \%$ & $3.8 \%$ & $15.8 \%$ & $27.2 \%$ & $41.1 \%$ & $8.9 \%$ \\
\hline $\begin{array}{l}\text { n. It is OK to walk off the official } \\
\text { trails, if I stay on unofficial trails } \\
\text { already created by visitors. } \\
\mathrm{N}=146 \text {, Mean=2.68 (Total } \\
\mathrm{N}=156 \text { ) }\end{array}$ & $20.5 \%$ & $27.6 \%$ & $17.9 \%$ & $16.7 \%$ & $10.9 \%$ & $6.4 \%$ \\
\hline
\end{tabular}

The next question deals with potential resource impacts at hiking areas in the New River Gorge, and if you perceive them to be a problem. Please place a check under the column that best describes to what extent each of the following is a problem for you when hiking at the New River Gorge. (check one box for each question)

\begin{tabular}{|l|c|c|c|c|c|c|}
\hline 18. & $\begin{array}{c}\text { Not a } \\
\text { problem }\end{array}$ & $\begin{array}{c}\text { Minor } \\
\text { problem }\end{array}$ & Neutral & $\begin{array}{c}\text { Moderate } \\
\text { problem }\end{array}$ & $\begin{array}{c}\text { Serious } \\
\text { problem }\end{array}$ & $\begin{array}{c}\text { Don't } \\
\text { know }\end{array}$ \\
\hline $\begin{array}{l}\text { a. } \text { Bare soil at the top or base of } \\
\text { cliffs. N=109, Mean=2.07 (Total } \\
\text { N=157) }\end{array}$ & $24.2 \%$ & $21 \%$ & $19.1 \%$ & $5.1 \%$ & $30.6 \%$ \\
\hline $\begin{array}{l}\text { b. Trampled vegetation at the top } \\
\text { or base of the cliff. N=124, } \\
\text { Mean=2.33 (Total N=158) }\end{array}$ & $18.4 \%$ & $23.4 \%$ & & $29.1 \%$ & $7.6 \%$ & $21.5 \%$ \\
\hline $\begin{array}{l}\text { c. Dead or damaged trees at the top } \\
\text { or base of the cliff due to use as a } \\
\text { climbing anchor, or rappel site. } \\
\text { N=123, Mean=2.49 (Total } \\
\text { N=157) }\end{array}$ & $18.5 \%$ & $20.4 \%$ & $22.3 \%$ & $17.2 \%$ & $21.7 \%$ \\
\hline $\begin{array}{l}\text { d. Erosion at/near the cliffs due to } \\
\text { multiple trails. N=125, } \\
\text { Mean=2.68 (Total N=157) }\end{array}$ & $12.7 \%$ & $19.1 \%$ & $16 \%$ & $28.7 \%$ & $19.1 \%$ & $20.4 \%$ \\
\hline $\begin{array}{l}\text { e. Vegetation damaged or being } \\
\text { removed from cliff face. N=121, } \\
\text { Mean=2.53 (Total N=156) }\end{array}$ & $19.9 \%$ & & $22.4 \%$ & $19.2 \%$ & $22.4 \%$ \\
\hline
\end{tabular}




\begin{tabular}{|c|c|c|c|c|c|}
\hline $\begin{array}{l}\text { f. Pieces of litter/trash at the cliff. } \\
\mathrm{N}=142 \text {, Mean }=2.52 \text { (Total } \\
\mathrm{N}=154 \text { ) }\end{array}$ & $27.9 \%$ & $20.8 \%$ & $11 \%$ & $32.5 \%$ & $7.8 \%$ \\
\hline $\begin{array}{l}\text { g. Human waste visible along cliff } \\
\text { lines or trails. } \mathrm{N}=137 \text {, } \\
\text { Mean }=2.26 \text { (Total } \mathrm{N}=151)\end{array}$ & $37.9 \%$ & $15.9 \%$ & $7.3 \%$ & $27.8 \%$ & $9.3 \%$ \\
\hline $\begin{array}{l}\text { h. Chalk marks on climbing routes. } \\
\mathrm{N}=109 \text {, Mean }=1.97 \text { (Total } \\
\mathrm{N}=156 \text { ) }\end{array}$ & $42.9 \%$ & $16 \%$ & $9 \%$ & $1.9 \%$ & $30.1 \%$ \\
\hline $\begin{array}{l}\text { i. Vandalism at NRG. } \mathrm{N}=126 \text {, } \\
\text { Mean }=2.41 \text { (Total } \mathrm{N}=157)\end{array}$ & $28 \%$ & $17.8 \%$ & $7.6 \%$ & $26.8 \%$ & $19.7 \%$ \\
\hline $\begin{array}{l}\text { j. People that do not control their } \\
\text { dogs adequately. } \mathrm{N}=136 \\
\text { Mean }=2.15 \text { (Total } \mathrm{N}=157)\end{array}$ & $34.4 \%$ & $22.3 \%$ & $12.7 \%$ & $17.2 \%$ & $13.4 \%$ \\
\hline
\end{tabular}

\section{Climbing is another popular activity in the NRG. This series of questions ask you to} think about your opinions about management preferences, as well as how hiking and climbing is currently managed at the New River Gorge. Please place a check under the column that best describes how strongly you agree or disagree with each of the following statements. (check one box for each question)

\section{Definitions:}

*Fixed Anchor: Presence of fixed bolts/webbing/pitons on cliff, used for anchor/rappel stations.

*Bolts: Permanent bolts used on sport routes, and as anchor points.

\begin{tabular}{|l|c|c|c|c|c|c|}
\hline 19. & $\begin{array}{c}\text { Strongly } \\
\text { disagree }\end{array}$ & Disagree & Neutral & Agree & $\begin{array}{c}\text { Strongly } \\
\text { Agree }\end{array}$ & $\begin{array}{c}\text { Don't } \\
\text { know }\end{array}$ \\
\hline $\begin{array}{l}\text { a. } \text { The impact of climbing bolts \& anchors } \\
\text { on the natural resource is minimal. } \\
\text { N=86, Mean=3.79 (Total N=143) }\end{array}$ & $1.4 \%$ & $4.9 \%$ & $13.3 \%$ & $25.9 \%$ & $14.7 \%$ & $39.9 \%$ \\
\hline $\begin{array}{l}\text { b. There should be official regulations } \\
\text { concerning where, when, and how bolts } \\
\text { and fixed anchors should be used. } \\
\text { N=105, Mean=3.95 (Total N=144) }\end{array}$ & $0.7 \%$ & $3.5 \%$ & $15.3 \%$ & $32.6 \%$ & $20.8 \%$ & $27.1 \%$ \\
\hline $\begin{array}{l}\text { c. } \text { The presence of numerous bolts \& } \\
\text { anchors negatively affects my hiking } \\
\text { experience. N=111, Mean=2.12 (Total } \\
\text { N=144) }\end{array}$ & $22.9 \%$ & $30.6 \%$ & $16.7 \%$ & $5.6 \%$ & $1.4 \%$ & $22.9 \%$ \\
\hline $\begin{array}{l}\text { d. The National Park Service managers at } \\
\text { the NRG share my values. N=72, } \\
\text { Mean=3.68 (Total N=144) }\end{array}$ & $0.7 \%$ & $0 \%$ & $21.5 \%$ & $20.1 \%$ & $7.6 \%$ & $50 \%$ \\
\hline $\begin{array}{l}\text { e. All climbing guide services should be } \\
\text { required to be certified or accredited. } \\
\text { N=120, Mean=4.2 (Total N=143) }\end{array}$ & $2.8 \%$ & $2.1 \%$ & $11.9 \%$ & $25.9 \%$ & $41.3 \%$ & $16.1 \%$ \\
\hline $\begin{array}{l}\text { f. I am familiar with the climbing } \\
\text { regulations at the NRG. N=93, } \\
\text { Mean=2.6 (Total N=144) }\end{array}$ & $13.9 \%$ & $21.5 \%$ & $11.8 \%$ & $11.1 \%$ & $6.3 \%$ & $35.4 \%$ \\
\hline $\begin{array}{l}\text { g. Climbing is not treated fairly in the } \\
\text { management process when compared to } \\
\text { other recreation activities at the NRG. }\end{array}$ & $0 \%$ & $9.8 \%$ & $18.9 \%$ & $4.2 \%$ & $0.7 \%$ & $66.4 \%$ \\
\hline
\end{tabular}




\begin{tabular}{|c|c|c|c|c|c|c|}
\hline $\mathrm{N}=48$, Mean $=2.88($ Total $\mathrm{N}=143)$ & & & & & & \\
\hline $\begin{array}{l}\text { h. Climbing group sizes should be limited } \\
\text { at NRG. } N=94 \text {, Mean=3.38 (Total } \\
N=142 \text { ) }\end{array}$ & $2.8 \%$ & $10.6 \%$ & $21.1 \%$ & $21.8 \%$ & $9.9 \%$ & $33.8 \%$ \\
\hline $\begin{array}{l}\text { i. I was not offended by seeing climbing } \\
\text { bolts or anchors. } \mathrm{N}=114 \text {, Mean }=1.68 \\
\text { (Total } \mathrm{N}=145)\end{array}$ & $42.1 \%$ & $23.4 \%$ & $11 \%$ & $0.7 \%$ & $1.4 \%$ & $21.4 \%$ \\
\hline $\begin{array}{l}\text { j. I trust that the National Park Service } \\
\text { managers at the } N R G \text { will do what is } \\
\text { right for the Park. } N=118 \text {, Mean }=4.07 \\
(\text { Total } N=145)\end{array}$ & $0.7 \%$ & $1.4 \%$ & $15.2 \%$ & $38.6 \%$ & $25.5 \%$ & $18.6 \%$ \\
\hline $\begin{array}{l}\text { k. Climbers need to be educated } \\
\text { concerning their role in the management } \\
\text { process. } \mathrm{N}=118 \text {, Mean }=4.22 \text { (Total } \\
\mathrm{N}=145 \text { ) }\end{array}$ & $2.8 \%$ & $0 \%$ & $6.2 \%$ & $40 \%$ & $32.4 \%$ & $18.6 \%$ \\
\hline $\begin{array}{l}\text { 1. Large groups of climbers detracted from } \\
\text { my experience at } \mathrm{NRG} . \mathrm{N}=114 \text {, } \\
\text { Mean }=1.98(\text { Total } \mathrm{N}=145)\end{array}$ & $29 \%$ & $29 \%$ & $15.9 \%$ & $2.8 \%$ & $2.1 \%$ & $21.4 \%$ \\
\hline $\begin{array}{l}\text { m. Most climbing areas need an official } \\
\text { management plan in order to provide } \\
\text { sustained use. } N=107, \text { Mean }=3.85 \\
(\text { Total } N=145)\end{array}$ & $3.4 \%$ & $4.1 \%$ & $14.5 \%$ & $29.7 \%$ & $22.1 \%$ & $26.2 \%$ \\
\hline $\begin{array}{l}\text { n. The National Park Service managers at } \\
\text { the NRG have the same goals as me. } \\
N=67, \text { Mean }=3.73 \text { (Total } N=143 \text { ) }\end{array}$ & $0 \%$ & $0.7 \%$ & $21 \%$ & $15.4 \%$ & $9.8 \%$ & $53.1 \%$ \\
\hline $\begin{array}{l}\text { o. Park managers do not have adequate } \\
\text { knowledge of climbing to properly } \\
\text { manage it at the NRG. } N=69 \text {, } \\
\text { Mean }=2.78 \text { (Total } N=143)\end{array}$ & $9.1 \%$ & $9.8 \%$ & $17.5 \%$ & $6.3 \%$ & $5.6 \%$ & $51.7 \%$ \\
\hline $\begin{array}{l}\text { p. Climber's practice of land stewardship } \\
\text { is not adequate. } \mathrm{N}=69, \text { Mean }=2.45 \\
\text { (Total } \mathrm{N}=142 \text { ) }\end{array}$ & $10.6 \%$ & $13.4 \%$ & $18.3 \%$ & $4.9 \%$ & $1.4 \%$ & $51.4 \%$ \\
\hline $\begin{array}{l}\text { q. Poor communication of rules \& } \\
\text { regulations is a problem at NRG. } \mathrm{N}=66 \text {, } \\
\text { Mean }=2.76(\text { Total } \mathrm{N}=142)\end{array}$ & $7 \%$ & $7 \%$ & $25.4 \%$ & $4.2 \%$ & $2.8 \%$ & $53.5 \%$ \\
\hline $\begin{array}{l}\text { r. Climbers have a negative attitude } \\
\text { toward management at the NRG. } \mathrm{N}=58 \text {, } \\
\text { Mean }=2.41(\text { Total } \mathrm{N}=143)\end{array}$ & $10.5 \%$ & $10.5 \%$ & $14 \%$ & $3.5 \%$ & $2.1 \%$ & $59.4 \%$ \\
\hline
\end{tabular}

20. These questions ask you to indicate how important the NRG is you. Please indicate to what extent you agree or disagree with the following statements about the New River Gorge area. (please check one box for each question)

\begin{tabular}{|l|c|c|c|c|c|c|}
\hline & $\begin{array}{c}\text { Strongly } \\
\text { Disagree }\end{array}$ & Disagree & Neutral & Agree & $\begin{array}{c}\text { Strongly } \\
\text { agree }\end{array}$ & $\begin{array}{c}\text { Don't } \\
\text { know }\end{array}$ \\
\hline $\begin{array}{l}\text { a. This place means a lot to me. N=159, } \\
\text { Mean=4.26 (Total N=159) }\end{array}$ & $1.3 \%$ & $1.3 \%$ & $16.4 \%$ & $32.1 \%$ & $49.1 \%$ & $0 \%$ \\
\hline b. I feel like this place is a part of me. & $4.4 \%$ & $8.8 \%$ & $35.2 \%$ & $24.5 \%$ & $26.4 \%$ & $0.6 \%$ \\
\hline
\end{tabular}




\begin{tabular}{|l|l|l|l|l|l|l|}
\hline N=158, Mean=3.6 (Total N=159) & & & & & & \\
\hline $\begin{array}{l}\text { c. I feel no commitment to this place. } \\
\text { N=154, Mean=2.02 (Total N=156) }\end{array}$ & $36.5 \%$ & $34.6 \%$ & $19.2 \%$ & $5.8 \%$ & $2.6 \%$ & $1.3 \%$ \\
\hline $\begin{array}{l}\text { d. I get more satisfaction out of hiking at } \\
\text { the New River Gorge than anywhere } \\
\text { else. N=157, Mean=3.15 (Total N=159) }\end{array}$ & $1.9 \%$ & $18.9 \%$ & $48.4 \%$ & $21.4 \%$ & $8.2 \%$ & $1.3 \%$ \\
\hline $\begin{array}{l}\text { e. The New River Gorge is the best place } \\
\text { for doing the kind of hiking I do. } \\
\mathrm{N}=153, \text { Mean=3.31 (Total N=158) }\end{array}$ & $1.9 \%$ & $10.1 \%$ & $52.5 \%$ & $20.9 \%$ & $11.4 \%$ & $3.2 \%$ \\
\hline $\begin{array}{l}\text { f. There is NO substitute for the type and } \\
\text { quality of hiking at the New River } \\
\text { Gorge. N=154, Mean=3.25 (Total } \\
\text { N=159) }\end{array}$ & $3.1 \%$ & $19.5 \%$ & $37.1 \%$ & $23.9 \%$ & $13.2 \%$ & $3.1 \%$ \\
\hline
\end{tabular}

21. How do you usually obtain information about hiking or other activities in the NRG? (check all sources that you have used) $\mathrm{N}=158$
$\square$ Local guidebook $30.4 \%$
$\square$ Hiking specific websites $43.7 \%$
$\square$ Friends/word of mouth $44.9 \% \square$ Through personal exploration \& discovery $43.7 \%$
$\square$ Outdoor stores around the NRG $11.4 \%$
$\square$ National Park Service information (website, brochure, etc.) $46.8 \%$
$\square$ Other: $5.6 \%$

22. Have you ever taken a course on, or been trained in, Leave No Trace (LNT) practices? (check one) $\mathrm{N}=159$
$\square$ Yes $23.9 \% \square$ No $76.1 \%$

23. Did you encounter any National Park Service rangers on the trails today? (check one ) N=159 $\square$ Yes $4.4 \% \quad \square$ No 95.6\%

24. How much do you trust or distrust the following as a source of information about cliff resource management? (please check for each item)

\begin{tabular}{|c|c|c|c|c|}
\hline & $\begin{array}{r}\text { Strongly } \\
\text { distrust }\end{array}$ & $\begin{array}{c}\text { Somewhat } \\
\text { distrust }\end{array}$ & $\begin{array}{c}\text { Somewhat } \\
\text { trust }\end{array}$ & $\begin{array}{c}\text { Strongly } \\
\text { trust }\end{array}$ \\
\hline Family \& friends $N=150$ & $4.7 \%$ & $12 \%$ & $57.3 \%$ & $26 \%$ \\
\hline Environmental organizations $\mathrm{N}=150$ & $3.3 \%$ & $2 \%$ & $51.3 \%$ & $43.3 \%$ \\
\hline Businesses (outdoor related) $\mathrm{N}=149$ & $2.7 \%$ & $16.1 \%$ & $57.7 \%$ & $23.5 \%$ \\
\hline Outdoor related organizations $\mathrm{N}=151$ & $0.7 \%$ & $4 \%$ & $54.3 \%$ & $41.1 \%$ \\
\hline Scientists in general $\mathrm{N}=149$ & $1.3 \%$ & $7.4 \%$ & $47.7 \%$ & $43.6 \%$ \\
\hline National Park rangers in general $\mathrm{N}=150$ & $0.7 \%$ & $4 \%$ & $38 \%$ & $57.3 \%$ \\
\hline $\begin{array}{l}\text { National Park rangers at the New River } \\
\text { Gorge National River } N=149\end{array}$ & $0.7 \%$ & $3.4 \%$ & $37.6 \%$ & $58.4 \%$ \\
\hline Mainstream news media $\mathrm{N}=150$ & $23.3 \%$ & $45.3 \%$ & $26.7 \%$ & $4.7 \%$ \\
\hline \multicolumn{5}{|c|}{$\begin{array}{l}\text { Of the sources listed above, which do you most strongly trust, and WHY? } \\
\text { List source: } \\
\text { Why trust?: }\end{array}$} \\
\hline
\end{tabular}


Of the sources listed above, which do you most strongly distrust, and WHY?

List source:

Why distrust?:

25. To which of the following organizations affiliated with the outdoors or the environment do you currently belong? (please check all that apply) $\mathrm{N}=43$

$\square$ Leave No Trace $20.9 \% \quad \square$ Nature Conservancy $20.9 \%$

$\square$ Sierra Club $18.6 \% \quad \square$ Wilderness Society $11.6 \%$

$\square$ National Audubon Society 16.3\% $\square$ National Parks Conservation Association 4.7\%

$\square$ Other (please write in) $32.6 \%$

26. Did you know that volunteers undertake trail maintenance projects at NRG?

(check one) $\mathrm{N}=155$

$\square$ Yes $40 \% \quad \square$ No $60 \%$

27. Have you ever volunteered for a trail maintenance project at NRG (trail construction, trash clean up, etc.)? (check one) $\mathrm{N}=154$

$\square$ Yes $11.7 \% \square$ No $88.3 \%$

28. Have you ever volunteered for trail maintenance project anywhere else (trail construction, trash clean up, etc.)? (check one) $\mathrm{N}=155$

$\square$ Yes $42.6 \% \square$ No $57.4 \%$

29. Do you think visitors should share responsibility for park resource (trails, etc.) maintenance? (check one) $\mathrm{N}=153$

$\square$ Yes $86.3 \% \square$ No $13.7 \%$

Finally, we would like to know a bit more about you.

30. What is your gender? (please check one) $\mathrm{N}=154$

$\square$ Male $50.6 \% \quad \square$ Female $49.4 \%$

31. What is the highest grade of school or year of college that you completed? (check one) $\mathrm{N}=156$

Less than high school $0.6 \%$

Four year college degree $35.9 \%$ 
High school graduate or GED 5.1\% $\square$ Masters, doctoral, or professional degree $39.1 \%$

Some college or associate degree 19.2\%

32. Are you Hispanic or Latino? (check one) $\mathrm{N}=155$

$\square$ Yes $1.9 \% \quad \square$ No $98.1 \%$

33. Which racial groups do you identify with? (check one or more) $\mathrm{N}=152$

$\square$ Native American or Alaska Native 2\%

Asian $2 \%$

Black or African American 1.3\%

$\square$ Native Hawaiian or other Pacific Islander 0.7\%

White $96.7 \%$

34. What is your age? (please write in number)

35. Are you a permanent resident or citizen of the U.S.? (check one and fill in) $\mathrm{N}=155$

$\square$ No - What is your country of origin? 2.6\%

$\square$ Yes — What is your zip code, city and state of residence? 97.4\%

Zip Code

City

State

36. How many people (including yourself) are in your party on this visit? (write in number)

36a. Which of the following best describes your personal group you are traveling with? (check one) $\mathrm{N}=155$

$\square$ Myself alone $11.6 \%$

$\square$ Myself with family (including spouse/partner and/or other family members/relatives) $38.7 \%$

Myself with family and friends $12.9 \%$

$\square$ Myself with friends $32.3 \%$

$\square$ Outfitter guided $1.3 \% \quad$ Specify

$\square$ Club/organization/school $3.2 \% \quad$ Specify

$\square$ Other (Please specify) 0\%

37. Do you consider yourself to be a leader or organizer for your group? $\mathrm{N}=156$
$\square$ Yes $44.2 \%$
$\square$ No $55.8 \%$

38. Are there any children under age 18 in your group? (check one \& write in number as needed) $\mathrm{N}=158$

$\square$ No $20.3 \%$ 
$\square$ Yes $\rightarrow$ How many children are in each of these age categories? 79.7\%

Number of children under 5 years old

Number of children age 5-12

Number of children age 13-17

\section{Thank you for your help!}

Privacy Act and Paperwork Reduction Act statement:

16 U.S.C. 1a-7 authorizes the National Park Service to collect this information to be used by park managers to better serve the public. The Paperwork Reduction Act states that your response to this request is voluntary. No action may be taken against you for refusing to supply the information requested. An agency may not conduct or sponsor, and a person is not required to respond to, a collection of information unless it displays a currently valid OMB control number and expiration date.

Burden estimate statement: Public reporting for this form is estimated to average 15 minutes per response. Direct comments regarding the burden estimate or any other aspect of this form to the Information Collection Clearance Officer, National Park Service, 1849 C Street, Washington, D.C. 20240. 
Appendix B: Climbing Survey Instrument

OMB Control Number 1024-0224

Expiration Date: 6/31/2011

\section{New River Gorge National River}

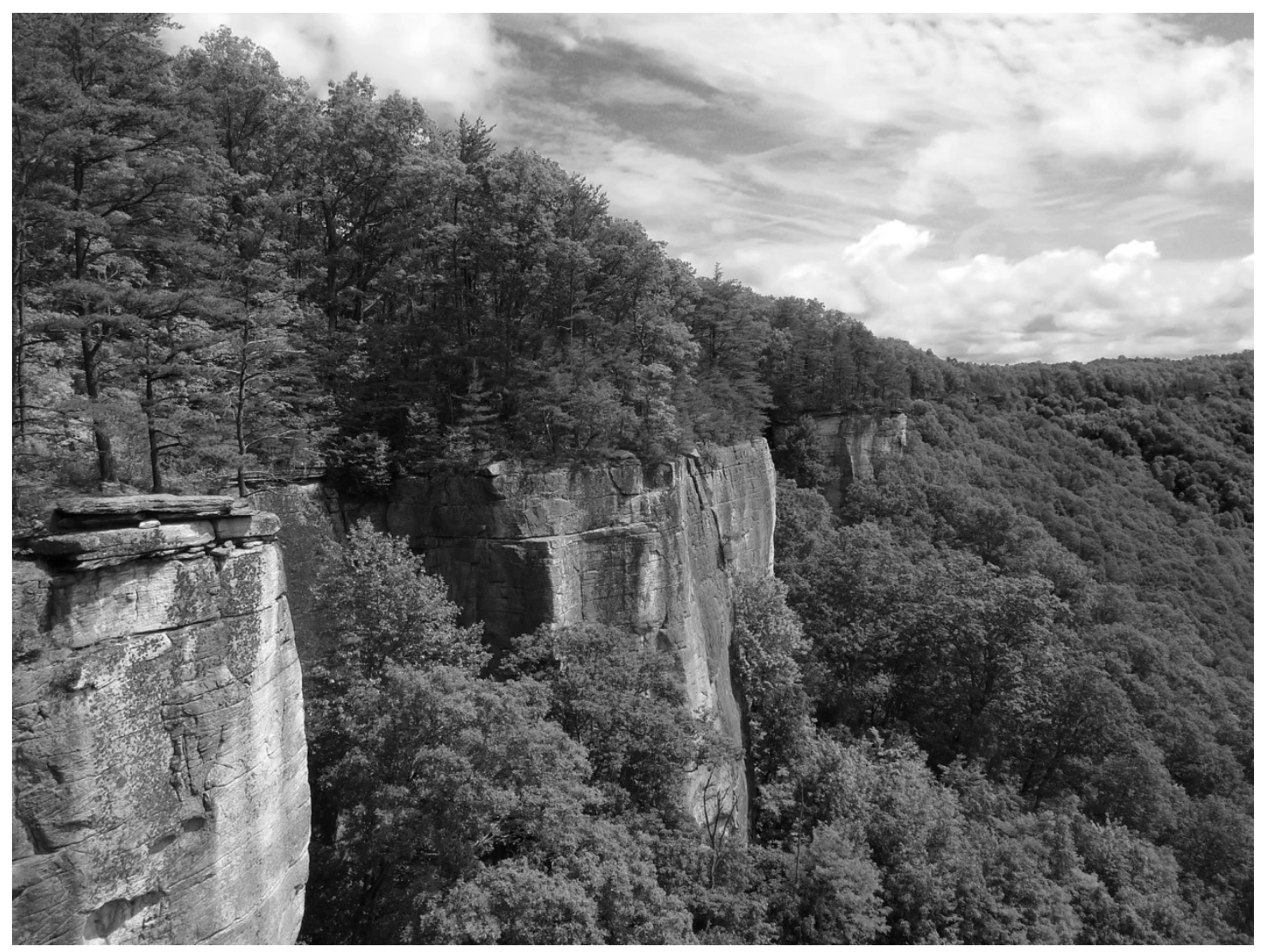

\section{A survey of visitors: Your opinion matters!}

\section{Conducted by:}

Dr. Dave Smaldone

Recreation, Parks \& Tourism Program

Division of Forestry \& Natural Resources

West Virginia University

In cooperation with the New River Gorge National River 


\section{Survey Directions:}

Thank you for taking the time to complete this survey. The results of this study will help resource managers better understand visitors like yourself, as well as develop education and interpretive programs for park visitors. This survey is only being given to a select number of visitors, so your participation is very important. Your participation is voluntary, and your responses will be kept confidential and anonymous. This survey should take about 20 minutes of your time. Please read the instructions for each question and answer to the best of your ability.

First, we would like to find out a little about your current visit to the New River Gorge National River.

1. Is this your first climbing trip to the New River Gorge (NRG)? (please check one) $\mathrm{N}=150$ $\square$ Yes $20 \% \quad \square$ No $80 \%$

2. How long is your current climbing trip? (please check one, and write in number) $\mathrm{N}=120$

$\square$ Less than one day. $27.5 \% \rightarrow$ If so, how many hours?

More than a day. $72.5 \% \rightarrow$ If so, how many days?

3. What activities are you doing today while on the trail? (check all that apply) $\mathrm{N}=149$

$\square$ hiking/walking $62.7 \%$

bird watching $6.7 \%$

$\square$ viewing wildlife (other than birds) $20 \%$

visiting historic sites $5.3 \%$

other: $4.7 \%$ $\square$ rock climbing $99.3 \%$

$\square$ photography $31.3 \%$

viewing the scenery $51.7 \%$

$\square$ picnicking $6 \%$

$\rightarrow$ Now, from the activities that you checked above, please write in the one that was most important to you: $\mathrm{N}=154$

Hiking/Walking 1.6\%

Rock Climbing $94.4 \%$

Photography $0.8 \%$

Viewing Scenery 0.8\%

Other $2.4 \%$

4. How many routes did you climb on this trip in the NRG? (please write in number)

5. How did the number of people you saw during your visit compare with what you expected? (please check one) $\mathrm{N}=149$

A lot less than what you expected $15.4 \%$

A little less than what you expected $20.8 \%$

About what you expected $47.7 \%$

A little more that what you expected $6.7 \%$

A lot more than what you expected $8.1 \%$

You did not have any expectations 1.3\% 
6. How crowded did you feel on your visit to the New River Gorge today? (circle one number) $\mathrm{N}=149$
$121.5 \%$
$225.5 \%$
$315.4 \%$
$411.4 \%$
$59.4 \%$
$610.1 \%$
$75.4 \%$
$81.3 \%$
$90 \%$

Not Crowded at all

Slightly Crowded

Moderately Crowded

Extremely Crowded

7. Did you move to a different crag today because of the number of people you encountered?

(please check one) $\mathrm{N}=148$

$\square$ Yes $9.5 \% \quad \square$ No $90.5 \%$

8. Would you consider climbing at a different crag based on the number of people you encountered? (please circle one response) $\mathrm{N}=149$
Not at all
Very Much
$128.2 \%$
$214.1 \%$
$34.7 \%$
$49.4 \%$
$522.8 \%$
$612.1 \%$
$78.7 \%$

9. How important to you are the following features at NRG climbing areas? (please circle one response for each question) $\mathrm{N}=149$

$$
\text { Not important } \quad \text { Very Important }
$$

\begin{tabular}{|c|c|c|c|c|c|c|}
\hline Restrooms at trailheads. & 2 & 3 & 4 & 5 & 6 & 7 Mean=3.64 \\
\hline Parking Areas.... & 2 & 3 & 4 & 5 & 6 & 7 Mean=5.66 \\
\hline Signage at Trail Access Points ..............1 & 2 & 3 & 4 & 5 & 6 & 7 Mean $=4.87$ \\
\hline Trail Quality............................ & 2 & 3 & 4 & 5 & 6 & 7 Mean=5.04 \\
\hline Visitor Center......... & 2 & 3 & 4 & 5 & 6 & 7 Mean $=2.15$ \\
\hline Ranger presence on trails. & 2 & 3 & 4 & 5 & 6 & 7 Mean $=2.05$ \\
\hline Suitable campsites in park. & 2 & 3 & 4 & 5 & 6 & 7 Mean $=4.26$ \\
\hline
\end{tabular}

10. How would you rate your satisfaction with the quality of these features at NRG climbing areas? (circle one response for each question)

$$
\begin{gathered}
\text { Very } \\
\text { Dissatisfied }
\end{gathered}
$$

Neutral

Very

Satisfied

Restrooms at trailheads.

Parking Areas

$\begin{array}{lllll}2 & 3 & 4 & 5 & 6 \\ 2 & 3 & 4 & 5 & 6 \\ 2 & 3 & 4 & 5 & 6 \\ 2 & 3 & 4 & 5 & 6 \\ 2 & 3 & 4 & 5 & 6 \\ 2 & 3 & 4 & 5 & 6 \\ 2 & 3 & 4 & 5 & 6\end{array}$

$7 \mathrm{~N}=147$, Mean $=4.46$

Signage at Trail Access Points...............

Trail Quality..............................1

Visitor Center..............................1

Ranger presence on trails. .................1

Suitable campsites in park. .................1

Now, we'd like you to tell us about your climbing background.

11. How many years have you been climbing? (write in number) 
12. How many years have you been climbing at the New River Gorge? (write in number)

13. When was your first visit to New River Gorge? (write in the month and year) Month: Year: _ $\square$ Don't know/can't remember

14. Approximately how many days a year do you climb outside? (please check one) $\mathrm{N}=150$
$\square<10$ days/year $12 \%$
$\square 31-40$ days/year $12.7 \%$
$\square$ 11-20 days/year $11.3 \%$
$\square$ 41-50 days/year $8.7 \%$
$\square$ 21-30 days/year $23.3 \%$
$\square>50$ days/year $32 \%$

15. Approximately how many days a year do you climb at the New River Gorge? N=147

$\square<10$ days/year $36.1 \% \quad \square 31-40$ days $/$ year $7.5 \%$

$\square$ 11-20 days/year $21.1 \% \quad \square$ 41-50 days/year $0.7 \%$

$\square$ 21-30 days/year $14.3 \% \quad \square>50$ days/year $20.4 \%$

16. Approximately how many days a year do you climb indoors in a gym? $\mathrm{N}=150$
$\square<10$ days/year $27.3 \%$
$\square$ 11-20 days/year $5.3 \%$
$\square 31-40$ days/year $9.3 \%$
$\square 21-30$ days/year $6.7 \%$
$\square$ 41-50 days/year 6\%
$\square>50$ days/year $45.3 \%$

17. What category best describes your overall ability as a climber? (check one) $\mathrm{N}=151$

$\square$ Novice $11.9 \% \quad \square$ Intermediate $43.7 \% \quad \square$ Advanced $37.1 \%$

$\square$ Expert $7.3 \%$

18. What is the hardest grade climb you have ever climbed in the NRG for each type of climbing below? (please write in grade number or check box)

Top roping: $\quad \square$ I do not Top Rope $\quad \square$ Don't know

Trad: $\square \square$ I do not Trad climb $\square$ Don't know

Sport: $\square$ I do not Sport climb $\square$ Don't know

19. What type of climbing did you do on this trip? (check all that apply) $\mathrm{N}=151$

$\square$ Top roping $69.5 \% \quad \square$ Bouldering $14.6 \%$

$\square$ Sport climbing $66.2 \% \quad \square$ Other $2 \%$

$\square$ Trad climbing $43 \%$

20. What type of climber are you predominately? (check one) $\mathrm{N}=152$

$\square$ Top roper $17.1 \% \quad \square$ Gym climber (indoors) $15.8 \%$

$\square$ Traditional $17.1 \% \quad \square$ Both sport \& traditional equally $18.4 \%$

$\square$ Sport (outdoors) 24.3\% $\square$ Boulderer 5.9\%

$\square$ Other: $1.3 \%$ 
21. Where did you learn to climb? (check one) $\mathrm{N}=151$

$\square$ Mostly inside at a rock gym $24.5 \%$

$\square$ Pretty evenly split, both inside at a gym, as well as outside $34.4 \%$

$\square$ Mostly outside $41.1 \%$

22. Who taught you to climb? (check all that apply) $\mathrm{N}=149$

$\square$ Family members (informally) $9.4 \%$

$\square$ Friends (informally) $71.8 \%$

$\square$ Climbing club (informally) $13.4 \%$

$\square$ Took a course from University/College (formal instruction) 10.1\%

$\square$ Took a course from outfitter/guide (formal instruction) $16.8 \%$

$\square$ Other: $10.7 \%$

22a. If you selected more than one option above, which one has had the most influence on your climbing knowledge and practices? (please write in your answer) $\mathrm{N}=32$

Family members $3.1 \%$

Friends 50\%

Climbing club $3.1 \%$

University/college course $6.3 \%$

Outfitter/guide course $31.3 \%$

Other $6.3 \%$

23. Where do you most prefer to climb? (check one) $\mathrm{N}=151$

$\square$ Gyms $4 \%$

$\square$ Front country (road-side crags, easy/short approaches) $57 \%$

$\square$ Back country (i.e. remote areas, long approaches) 39\%

24. From your home, how many hours did you travel to reach the New River Gorge? (please check one box) $\mathrm{N}=152$

$\square$ Less than $114.5 \%$

7 to $812.5 \%$

$\square 1$ to $28.6 \% \quad \square 3$ to $423.7 \% \quad \square 5$ to $626.3 \%$

$\square$ more than $814.5 \%$

25. Please indicate which climbing areas you typically use at the NRG: (check all that apply) $\mathrm{N}=151$

$\square$ Bridge Buttress 62.3\% $\square$ Bubba City 49\% $\square$ Kaymoor 55.6\%

$\square$ Endless Wall 57.6\% $\square$ Beauty Mtn. 36.4\% $\square$ South Nuttall 7.9\%

$\square$ Junkyard Wall 56.3\% $\square$ Fern Buttress 37.7\%

$\square$ Don't Know Name of Site/Crag 5.3\% 
26. What would you be most interested in learning about, in regards to the cliffs and their ecosystems at the NRG? (please write in your answer)

The following questions deal with the cliffs at the NRG and your opinion of their importance. Please place a check under the column that best describes how strongly you agree or disagree with each of the following statements. (check one box for each question)

\begin{tabular}{|l|c|c|c|c|c|c|}
\hline 27. & $\begin{array}{c}\text { Strongly } \\
\text { Disagree }\end{array}$ & Disagree & Neutral & Agree & $\begin{array}{c}\text { Strongly } \\
\text { agree }\end{array}$ & $\begin{array}{c}\text { Don't } \\
\text { know }\end{array}$ \\
\hline $\begin{array}{l}\text { a. The cliffs in the NRG are the same } \\
\text { as cliffs anywhere else. N=147, } \\
\text { Mean=1.76 (Total N=151) }\end{array}$ & $39.7 \%$ & $43.7 \%$ & $11.9 \%$ & $1.3 \%$ & $0.7 \%$ & $2.6 \%$ \\
\hline $\begin{array}{l}\text { b. Cliffs are a fundamental resource to } \\
\text { the NRG National River, and } \\
\text { essential to its national significance. } \\
\text { N=150, Mean=4.63 (Total N=151) }\end{array}$ & $2.6 \%$ & $1.3 \%$ & $4.6 \%$ & $13.2 \%$ & $77.5 \%$ & $0.7 \%$ \\
\hline $\begin{array}{l}\text { c. The cliffs in the NRG provide } \\
\text { habitat for rare plants. N=112, } \\
\text { Mean=3.9 (Total N=150) }\end{array}$ & $2 \%$ & $2.7 \%$ & $17.3 \%$ & $31.3 \%$ & $21.3 \%$ & $25.3 \%$ \\
\hline $\begin{array}{l}\text { d. The cliffs in the NRG provide } \\
\text { habitat for rare animals. N=110, } \\
\text { Mean=3.92 (Total N=149) }\end{array}$ & $1.3 \%$ & $3.4 \%$ & $16.8 \%$ & $30.9 \%$ & $21.5 \%$ & $26.2 \%$ \\
\hline $\begin{array}{l}\text { e. The cliffs in the NRG support } \\
\text { unique habitats and ecosystems. } \\
\text { N=124, Mean=4.1 (Total N=149) }\end{array}$ & $2 \%$ & $2.7 \%$ & $11.4 \%$ & $36.2 \%$ & $30.9 \%$ & $16.8 \%$ \\
\hline $\begin{array}{l}\text { f. The views of natural landscape } \\
\text { from the cliffs in the NRG are } \\
\text { worth protecting from human } \\
\text { development. N=149, Mean=4.61 } \\
\text { (Total N=151) }\end{array}$ & $2.6 \%$ & $0.7 \%$ & $7.3 \%$ & $11.3 \%$ & $76.8 \%$ & $1.3 \%$ \\
\hline
\end{tabular}




\begin{tabular}{|l|l|l|l|l|l|l|}
\hline $\begin{array}{l}\text { g. The views from the cliffs in the } \\
\text { NRG are one of the Park's most } \\
\text { important and unique resources. } \\
\text { N=149, Mean=4.45 (Total N=151) }\end{array}$ & $2.6 \%$ & $2 \%$ & $8.6 \%$ & $20.5 \%$ & $64.9 \%$ & $1.3 \%$ \\
\hline
\end{tabular}

This next section has questions about your behaviors and opinions regarding climbing practices. Please place a check under the column that best describes how strongly you agree or disagree with each of the following statements. (check one box for each question)

\section{Definitions:}

*Fixed Anchor: Presence of fixed bolts/webbing/pitons on cliff, used for anchor/rappel stations.

*Bolts: Permanent bolts used on sport routes, and as anchor points.

\begin{tabular}{|l|c|c|c|c|c|c|}
\hline 28. & $\begin{array}{c}\text { Strongly } \\
\text { Disagree }\end{array}$ & Disagree & Neutral & Agree & $\begin{array}{c}\text { Strongly } \\
\text { agree }\end{array}$ & $\begin{array}{c}\text { Don't } \\
\text { know }\end{array}$ \\
\hline $\begin{array}{l}\text { a. I always pack out my trash. N=151, } \\
\text { Mean=4.89 (Total N=151) }\end{array}$ & $0.7 \%$ & $0 \%$ & $1.3 \%$ & $6 \%$ & $92.1 \%$ & $0 \%$ \\
\hline $\begin{array}{l}\text { b. I always use official access trails. } \\
\text { N=150, Mean=4.37 (Total N=151) }\end{array}$ & $0.7 \%$ & $2.6 \%$ & $11.3 \%$ & $29.8 \%$ & $55 \%$ & $0.7 \%$ \\
\hline $\begin{array}{l}\text { c. I respect revegetation efforts. N=149, } \\
\text { Mean=4.7 (Total N=151) }\end{array}$ & $0 \%$ & $0 \%$ & $2 \%$ & $25.2 \%$ & $71.5 \%$ & $1.3 \%$ \\
\hline $\begin{array}{l}\text { d. I avoid trails that have been closed. } \\
\text { N=150, Mean=4.56 (Total N=151) }\end{array}$ & $0 \%$ & $2 \%$ & $6.6 \%$ & $24.5 \%$ & $66.2 \%$ & $0.7 \%$ \\
\hline $\begin{array}{l}\text { e. I practice Leave No Trace when I } \\
\text { climb. N=149, Mean=4.75 (Total } \\
\text { N=151) }\end{array}$ & $0 \%$ & $0 \%$ & $4 \%$ & $16.6 \%$ & $78.1 \%$ & $1.3 \%$ \\
\hline $\begin{array}{l}\text { f. Fixed anchors (permanent) should be } \\
\text { used to help preserve the environment. } \\
\text { N=148, Mean=4.66 (Total N=151) }\end{array}$ & $0 \%$ & $1.3 \%$ & $4 \%$ & $21.9 \%$ & $70.9 \%$ & $2 \%$ \\
\hline $\begin{array}{l}\text { g. I comply with local climbing } \\
\text { regulations. N=148, Mean=4.7 (Total } \\
\text { N=151) }\end{array}$ & $0 \%$ & $0.7 \%$ & $2.6 \%$ & $22.5 \%$ & $72.2 \%$ & $2 \%$ \\
\hline $\begin{array}{l}\text { h. I try to keep my noise level down. } \\
\text { N=150, Mean=4.4 (Total N=150) }\end{array}$ & $0 \%$ & $2 \%$ & $9.9 \%$ & $33.8 \%$ & $54.3 \%$ & $0 \%$ \\
\hline $\begin{array}{l}\text { i. Old gear should be removed from } \\
\text { belay and rappel sites. N=150, } \\
\text { Mean=4.51 (Total N=151) }\end{array}$ & $0 \%$ & $1.3 \%$ & $7.9 \%$ & $28.5 \%$ & $61.6 \%$ & $0.7 \%$ \\
\hline $\begin{array}{l}\text { j. Permanent anchors provide easy access } \\
\text { to routes. N=151, Mean=4.56 (Total } \\
\text { N=151) }\end{array}$ & $0 \%$ & $2 \%$ & $6.6 \%$ & $24.5 \%$ & $66.9 \%$ & $0 \%$ \\
\hline $\begin{array}{l}\text { k. I park my vehicle in designated areas. } \\
\text { N=150, Mean=4.71 (Total N=151) }\end{array}$ & $0 \%$ & $0 \%$ & $3 \%$ & $24.5 \%$ & $72.8 \%$ & $0.7 \%$ \\
\hline $\begin{array}{l}\text { 1. I always consider the local area ethics } \\
\text { when I climb. N=149, Mean=4.66 } \\
\text { (Total N=151) }\end{array}$ & $0 \%$ & $0 \%$ & $25.8 \%$ & $68.9 \%$ & $1.3 \%$ \\
\hline $\begin{array}{l}\text { m. Permanent bolted anchors should be } \\
\text { used at the top of climbs. N=151, } \\
\text { Mean=4.46 (Total N=151) }\end{array}$ & $0 \%$ & $1.3 \%$ & $9.9 \%$ & $29.8 \%$ & $58.9 \%$ & $0 \%$ \\
\hline
\end{tabular}




\begin{tabular}{|c|c|c|c|c|c|c|}
\hline $\begin{array}{l}\text { n. It is OK to remove/clean lichens. } \\
\qquad \mathrm{N}=144, \text { Mean=2.88 (Total } \mathrm{N}=149)\end{array}$ & $15.4 \%$ & $18.1 \%$ & $34.9 \%$ & $18.8 \%$ & $9.41 \%$ & $3.4 \%$ \\
\hline $\begin{array}{l}\text { o. It is OK to remove/clean mosses and } \\
\text { other vegetation. } \mathrm{N}=145, \text { Mean }=2.7 \\
\text { (Total } \mathrm{N}=149 \text { ) }\end{array}$ & $20.1 \%$ & $21.5 \%$ & $31.5 \%$ & $16.1 \%$ & $8.1 \%$ & $2.7 \%$ \\
\hline $\begin{array}{l}\text { p. Chiseling holds is an acceptable } \\
\text { practice. } \mathrm{N}=140 \text {, Mean=1.37 (Total } \\
\mathrm{N}=148 \text { ) }\end{array}$ & $71.6 \%$ & $13.5 \%$ & $8.1 \%$ & $0 \%$ & $1.4 \%$ & $5.4 \%$ \\
\hline $\begin{array}{l}\text { q. It is OK to secure top ropes to trees. } \\
\mathrm{N}=149, \text { Mean }=3.07 \text { (Total } \mathrm{N}=151)\end{array}$ & $12.6 \%$ & $17.2 \%$ & $28.5 \%$ & $31.8 \%$ & $8.6 \%$ & $1.3 \%$ \\
\hline $\begin{array}{l}\text { r. Permanent anchors increase the use of } \\
\text { routes too much. } \mathrm{N}=144 \text {, Mean }=2.33 \\
(\text { Total } \mathrm{N}=150)\end{array}$ & $20 \%$ & $38.7 \%$ & $26 \%$ & $8.7 \%$ & $2.7 \%$ & $4 \%$ \\
\hline $\begin{array}{l}\text { s. Soils \& plants along the Park trails are } \\
\text { hardy and not easily damaged. } \mathrm{N}=141 \text {, } \\
\text { Mean }=2.28(\text { Total } \mathrm{N}=150)\end{array}$ & $16 \%$ & $46.7 \%$ & $22.7 \%$ & $6.7 \%$ & $2 \%$ & $6 \%$ \\
\hline $\begin{array}{l}\text { t. I respect seasonal closures at certain } \\
\text { crags for wildlife needs. } \mathrm{N}=147 \text {, } \\
\text { Mean }=4.41(\text { Total } \mathrm{N}=151)\end{array}$ & $0.7 \%$ & $3.3 \%$ & $5.3 \%$ & $33.8 \%$ & $54.3 \%$ & $2.6 \%$ \\
\hline $\begin{array}{l}\text { u. The park provides clear indications } \\
\text { about which trails should not be used. } \\
\mathrm{N}=142 \text {, Mean }=3.39 \text { (Total } \mathrm{N}=151)\end{array}$ & $4 \%$ & $15.2 \%$ & $30.5 \%$ & $28.5 \%$ & $15.9 \%$ & $6 \%$ \\
\hline $\begin{array}{l}\text { v. Compared to other people I saw on } \\
\text { this visit, I am causing less damage to } \\
\text { the soils \& vegetation. } N=135 \text {, } \\
\text { Mean }=3.53 \text { (Total } N=151)\end{array}$ & $0.7 \%$ & $1.3 \%$ & $51.7 \%$ & $21.9 \%$ & $13.9 \%$ & $10.6 \%$ \\
\hline $\begin{array}{l}\text { w. Permanent anchors are not needed. } \\
\mathrm{N}=146, \text { Mean }=1.8(\text { Total } \mathrm{N}=151)\end{array}$ & $41.1 \%$ & $38.4 \%$ & $13.9 \%$ & $2 \%$ & $1.3 \%$ & $3.3 \%$ \\
\hline $\begin{array}{l}\text { x. I feel a responsibility to help protect } \\
\text { the plants and ecosystems in the Park. } \\
\mathrm{N}=148 \text {, Mean }=4.33 \text { (Total } \mathrm{N}=151)\end{array}$ & $1.3 \%$ & $1.3 \%$ & $6 \%$ & $44.4 \%$ & $45 \%$ & $2 \%$ \\
\hline $\begin{array}{l}\text { y. The continued use of unofficial visitor } \\
\text { created trails is a serious threat to } \\
\text { plants and soils along the Park trails. } \\
\mathrm{N}=140 \text {, Mean=3.7 (Total } \mathrm{N}=151 \text { ) }\end{array}$ & $3.3 \%$ & $11.3 \%$ & $19.2 \%$ & $35.1 \%$ & $23.8 \%$ & $7.3 \%$ \\
\hline $\begin{array}{l}\text { z. It is OK to walk off the official trails, } \\
\text { if I stay on unofficial trails already } \\
\text { created by visitors. } N=145 \text {, Mean }=2.41 \\
\text { (Total } N=150 \text { ) }\end{array}$ & $20 \%$ & $33.3 \%$ & $31.3 \%$ & $8 \%$ & $4 \%$ & $3.3 \%$ \\
\hline
\end{tabular}

The next section deals with potential resource impacts at climbing areas in the New River Gorge, and if you perceive them to be a problem. Please place a check under the column that best describes to what extent each of the following is a problem for you when climbing at the New River Gorge. (check one box for each question)

\begin{tabular}{|l|c|c|c|c|c|}
\hline 29. & $\begin{array}{c}\text { Not a } \\
\text { problem }\end{array}$ & $\begin{array}{c}\text { Minor } \\
\text { problem }\end{array}$ & $\begin{array}{c}\text { Moderate } \\
\text { problem }\end{array}$ & $\begin{array}{c}\text { Serious } \\
\text { problem }\end{array}$ & $\begin{array}{c}\text { Don't } \\
\text { know }\end{array}$ \\
\hline $\begin{array}{c}\text { a. Bare soil at the top or base of } \\
\text { cliffs. N=131, Mean=2.3 (Total }\end{array}$ & $22.7 \%$ & $24.7 \%$ & $31.3 \%$ & $8.7 \%$ & $12.7 \%$ \\
\hline
\end{tabular}




\begin{tabular}{|l|l|l|l|l|l|}
\hline N=150) & & & & & \\
\hline $\begin{array}{l}\text { b. Trampled vegetation at the top } \\
\text { or base of the cliff. N=135, } \\
\text { Mean=2.38 (Total N=150) }\end{array}$ & $17.3 \%$ & $32 \%$ & $30 \%$ & $10.7 \%$ & $10 \%$ \\
\hline $\begin{array}{l}\text { c. Dead or damaged trees at the top } \\
\text { or base of the cliff due to use as } \\
\text { an anchor, or rappel site. N=130, } \\
\text { Mean=2.7 (Total N=150) }\end{array}$ & $16.7 \%$ & $18.7 \%$ & $32 \%$ & $24.7 \%$ & $8 \%$ \\
\hline $\begin{array}{l}\text { d. Erosion at/near the cliffs due to } \\
\text { multiple trails. N=141, } \\
\text { Mean=2.7 (Total N=150) }\end{array}$ & $12 \%$ & $25.3 \%$ & $36 \%$ & $20.7 \%$ & $6 \%$ \\
\hline $\begin{array}{l}\text { e. Vegetation damaged or being } \\
\text { removed from cliff face. N=136, } \\
\text { Mean=2.18 (Total N=149) }\end{array}$ & $24.8 \%$ & $34.2 \%$ & $22.8 \%$ & $9.4 \%$ & $8.7 \%$ \\
\hline $\begin{array}{l}\text { f. Pieces of litter/trash at the cliff. } \\
\text { N=145, Mean=2.81 (Total } \\
\text { N=147) }\end{array}$ & $11.6 \%$ & $29.3 \%$ & $23.8 \%$ & $34 \%$ & $1.4 \%$ \\
\hline $\begin{array}{l}\text { g. Human waste visible along cliff } \\
\text { lines or trails. N=142, } \\
\text { Mean=2.46 (Total N=150) }\end{array}$ & $28.7 \%$ & $23.3 \%$ & $13.3 \%$ & $29.3 \%$ & $5.3 \%$ \\
\hline $\begin{array}{l}\text { h. Chalk tick marks on routes. } \\
\text { N=147, Mean=1.67 (Total=149) }\end{array}$ & $53.7 \%$ & $27.5 \%$ & $14.1 \%$ & $3.4 \%$ & $1.3 \%$ \\
\hline $\begin{array}{l}\text { i. Vandalism at NRG. N=137, } \\
\text { Mean=2.43 (Total N=149) }\end{array}$ & $30.2 \%$ & $18.1 \%$ & $17.4 \%$ & $26.2 \%$ & $8.1 \%$ \\
\hline $\begin{array}{l}\text { j. People that do not control their } \\
\text { dogs adequately. N=144, } \\
\text { Mean=2.41 (Total N=150) }\end{array}$ & $27.3 \%$ & $20.7 \%$ & $29.3 \%$ & $18.7 \%$ & $4 \%$ \\
\hline
\end{tabular}

This series of questions ask you to think about your opinions about management preferences, as well as how climbing is currently managed at the New River Gorge. Please place a check under the column that best describes how strongly you agree or disagree with each of the following statements. (check one box for each question)

\begin{tabular}{|l|c|c|c|c|c|c|}
\hline 30. & $\begin{array}{c}\text { Strongly } \\
\text { disagree }\end{array}$ & Disagree & Neutral & Agree & $\begin{array}{c}\text { Strongly } \\
\text { Agree }\end{array}$ & $\begin{array}{c}\text { Don't } \\
\text { know }\end{array}$ \\
\hline $\begin{array}{l}\text { a. The impact of bolts \& anchors on the } \\
\text { natural resource is minimal. N=149, } \\
\text { Mean=4.03 (Total N=151) }\end{array}$ & $1.3 \%$ & $5.3 \%$ & $13.9 \%$ & $47 \%$ & $31.1 \%$ & $1.3 \%$ \\
\hline $\begin{array}{l}\text { b. There should be official regulations } \\
\text { concerning where, when, and how bolts } \\
\text { and fixed anchors should be used. } \\
\text { N=147, Mean=3.64 (Total N=151) }\end{array}$ & $4.6 \%$ & $9.3 \%$ & $23.8 \%$ & $38.4 \%$ & $21.2 \%$ & $2.6 \%$ \\
\hline $\begin{array}{l}\text { c. The presence of numerous bolts \& } \\
\text { anchors negatively affects my climbing } \\
\text { experience. N=150, Mean=1.9 (Total } \\
\text { N=151) }\end{array}$ & $39.7 \%$ & $37.7 \%$ & $13.9 \%$ & $7.9 \%$ & $0 \%$ & $0.7 \%$ \\
\hline $\begin{array}{l}\text { d. The National Park Service managers at } \\
\text { the NRG share my values. N=97, }\end{array}$ & $4.7 \%$ & $5.3 \%$ & $33.3 \%$ & $17.3 \%$ & $4 \%$ & $35.3 \%$ \\
\hline
\end{tabular}




\begin{tabular}{|c|c|c|c|c|c|c|}
\hline Mean $=3.16($ Total $N=150)$ & & & & & & \\
\hline $\begin{array}{l}\text { e. All climbing guide services should be } \\
\text { required to be certified or accredited. } \\
\mathrm{N}=146, \text { Mean }=4.22 \text { (Total } \mathrm{N}=149)\end{array}$ & $1.3 \%$ & $6.7 \%$ & $12.7 \%$ & $25.3 \%$ & $51.3 \%$ & $2.7 \%$ \\
\hline $\begin{array}{l}\text { f. I am familiar with the climbing } \\
\text { regulations at the } \mathrm{NRG} . \mathrm{N}=142 \text {, } \\
\text { Mean }=3.75(\text { Total } \mathrm{N}=150)\end{array}$ & $2.7 \%$ & $12 \%$ & $13.3 \%$ & $44.7 \%$ & $22 \%$ & $5.3 \%$ \\
\hline $\begin{array}{l}\text { g. Climbing is not treated fairly in the } \\
\text { management process when compared to } \\
\text { other recreation activities at the NRG. } \\
\mathrm{N}=102 \text {, Mean=3.15 (Total } \mathrm{N}=151 \text { ) }\end{array}$ & $0.7 \%$ & $16.6 \%$ & $29.1 \%$ & $14.6 \%$ & $6.6 \%$ & $32.5 \%$ \\
\hline $\begin{array}{l}\text { h. Climbing group sizes should be limited } \\
\text { at } N R G \text {. } N=145 \text {, Mean=3.06 (Total } \\
N=151 \text { ) }\end{array}$ & $9.3 \%$ & $31.3 \%$ & $19.9 \%$ & $16.6 \%$ & $19.2 \%$ & $4 \%$ \\
\hline $\begin{array}{l}\text { i. Other recreational groups are not } \\
\text { offended by seeing climbing bolts or } \\
\text { anchors. } \mathrm{N}=111 \text {, Mean }=1.97 \text { (Total } \\
\mathrm{N}=151 \text { ) }\end{array}$ & $25.8 \%$ & $27.8 \%$ & $16.6 \%$ & $2.6 \%$ & $0.7 \%$ & $26.5 \%$ \\
\hline $\begin{array}{l}\text { j. I trust that the National Park Service } \\
\text { managers at the NRG will do what is } \\
\text { right for the Park. } N=129 \text {, Mean }=3.43 \\
(\text { Total } N=151)\end{array}$ & $5.3 \%$ & $11.3 \%$ & $20.5 \%$ & $37.7 \%$ & $10.6 \%$ & $14.6 \%$ \\
\hline $\begin{array}{l}\text { k. Climbers need to be educated } \\
\text { concerning their role in the management } \\
\text { process. } \mathrm{N}=146 \text {, Mean }=4.12 \text { (Total } \\
\mathrm{N}=151 \text { ) }\end{array}$ & $0 \%$ & $2.6 \%$ & $15.2 \%$ & $46.4 \%$ & $32.5 \%$ & $3.3 \%$ \\
\hline $\begin{array}{l}\text { 1. Large groups of climbers detracted from } \\
\text { my experience at } N R G . N=149 \text {, } \\
\text { Mean }=2.83 \text { (Total } N=151)\end{array}$ & $12.6 \%$ & $31.8 \%$ & $23.2 \%$ & $21.9 \%$ & $9.3 \%$ & $1.3 \%$ \\
\hline $\begin{array}{l}\text { m. Most climbing areas need an official } \\
\text { management plan in order to provide } \\
\text { sustained use. } N=141 \text {, Mean }=3.47 \\
(\text { Total } N=151)\end{array}$ & $5.3 \%$ & $10.6 \%$ & $25.8 \%$ & $38.4 \%$ & $13.2 \%$ & $6.6 \%$ \\
\hline $\begin{array}{l}\text { n. The National Park Service managers at } \\
\text { the NRG have the same goals as me. } \\
\mathrm{N}=91 \text {, Mean }=2.99 \text { (Total } \mathrm{N}=149)\end{array}$ & $5.4 \%$ & $11.4 \%$ & $26.8 \%$ & $13.4 \%$ & $4 \%$ & $38.9 \%$ \\
\hline $\begin{array}{l}\text { o. Park managers do not have adequate } \\
\text { knowledge of climbing to properly } \\
\text { manage it at the NRG. } \mathrm{N}=100 \text {, } \\
\text { Mean=3.2 (Total } \mathrm{N}=149)\end{array}$ & $3.4 \%$ & $10.7 \%$ & $28.2 \%$ & $18.8 \%$ & $6 \%$ & $32.9 \%$ \\
\hline $\begin{array}{l}\text { p. Climber's practice of land stewardship } \\
\text { is not adequate. } \mathrm{N}=128 \text {, Mean }=2.6 \\
\text { (Total } \mathrm{N}=149 \text { ) }\end{array}$ & $12.8 \%$ & $26.8 \%$ & $31.5 \%$ & $11.4 \%$ & $3.4 \%$ & $14.1 \%$ \\
\hline $\begin{array}{l}\text { q. Poor communication of rules } \& \\
\text { regulations is a problem at NRG. } \\
\mathrm{N}=116, \text { Mean }=2.99(\text { Total } \mathrm{N}=149)\end{array}$ & $4 \%$ & $18.8 \%$ & $33.6 \%$ & $16.8 \%$ & $4.7 \%$ & $22.1 \%$ \\
\hline $\begin{array}{l}\text { r. Climbers have a negative attitude } \\
\text { toward management at the NRG. } \\
\mathrm{N}=101, \text { Mean }=2.7 \text { (Total } \mathrm{N}=149)\end{array}$ & $8.7 \%$ & $22.1 \%$ & $22.1 \%$ & $10.1 \%$ & $4.7 \%$ & $32.2 \%$ \\
\hline
\end{tabular}


31. These questions ask you to indicate how important the NRG is you. Please indicate to what extent you agree or disagree with the following statements about the New River Gorge area.

(Please check one box for each question)

\begin{tabular}{|l|c|c|c|c|c|c|}
\hline & $\begin{array}{c}\text { Strongly } \\
\text { Disagree }\end{array}$ & Disagree & Neutral & Agree & $\begin{array}{c}\text { Strongly } \\
\text { agree }\end{array}$ & $\begin{array}{c}\text { Don't } \\
\text { know }\end{array}$ \\
\hline $\begin{array}{l}\text { a. This place means a lot to me. N=150, } \\
\text { Mean=4.67 (Total N=151) }\end{array}$ & $0 \%$ & $0 \%$ & $6.6 \%$ & $19.9 \%$ & $72.8 \%$ & $0.7 \%$ \\
\hline $\begin{array}{l}\text { b. I feel like this place is a part of me. } \\
\text { N=148, Mean=4.19 (Total N=151) }\end{array}$ & $0.7 \%$ & $7.9 \%$ & $14.6 \%$ & $23.8 \%$ & $51 \%$ & $2 \%$ \\
\hline $\begin{array}{l}\text { c. I feel no commitment to this place. } \\
\text { N=148, Mean=1.59 (Total N=149) }\end{array}$ & $65.1 \%$ & $20.8 \%$ & $6 \%$ & $4 \%$ & $3.4 \%$ & $0.7 \%$ \\
\hline $\begin{array}{l}\text { d. I get more satisfaction out of climbing } \\
\text { at the New River Gorge than anywhere } \\
\text { else. N=147, Mean=3.63 (Total N=150) }\end{array}$ & $0 \%$ & $15.2 \%$ & $33.1 \%$ & $21.9 \%$ & $27.2 \%$ & $2.6 \%$ \\
\hline $\begin{array}{l}\text { e. The New River Gorge is the best place } \\
\text { for doing the kind of climbing I do. } \\
\text { N=150, Mean=3.66 (Total N=151) }\end{array}$ & $0.7 \%$ & $13.2 \%$ & $31.1 \%$ & $28.5 \%$ & $25.8 \%$ & $0.7 \%$ \\
\hline $\begin{array}{l}\text { f. There is NO substitute for the type and } \\
\text { quality of climbing at the New River } \\
\text { Gorge. N=147, Mean=3.9 (Total } \\
\text { N=151) }\end{array}$ & $5.3 \%$ & $8.6 \%$ & $19.9 \%$ & $19.9 \%$ & $43.7 \%$ & $2.6 \%$ \\
\hline
\end{tabular}

32. How do you usually obtain information about climbing in the NRG? (check all sources that you have used) $\mathrm{N}=148$

$\square$ Local guidebook $87.2 \%$

$\square$ Friends/word of mouth $77.9 \%$

$\square$ Climbing websites $44.3 \%$

$\square$ Through personal exploration \& discovery $39.8 \%$

$\square$ Outdoor/climbing stores around the NRG 38.9\%

$\square$ National Park Service information (website, brochure, etc.) $9.4 \%$

$\square$ Other: $2 \%$

33. Have you ever taken a course on, or been trained in, Leave No Trace (LNT) climbing practices? (check one) $\mathrm{N}=151$

$\square$ Yes $37.1 \% \square$ No $62.9 \%$

34. Did you encounter any National Park Service rangers on the trails today? $N=151$

$\square$ Yes $1.3 \% \quad \square$ No $98.7 \%$

35. How much do you trust or distrust the following as a source of information about climbing management? (please place a check in the appropriate box)

\begin{tabular}{|l|c|c|c|c|}
\hline & $\begin{array}{c}\text { Strongly } \\
\text { distrust }\end{array}$ & $\begin{array}{c}\text { Somewhat } \\
\text { distrust }\end{array}$ & $\begin{array}{c}\text { Somewhat } \\
\text { trust }\end{array}$ & $\begin{array}{c}\text { Strongly } \\
\text { trust }\end{array}$ \\
\hline Family \& friends $\mathrm{N}=147$ & $2 \%$ & $6.1 \%$ & $46.9 \%$ & $44.9 \%$ \\
\hline
\end{tabular}




\begin{tabular}{|c|c|c|c|c|}
\hline Environmental organizations $\mathrm{N}=146$ & $2.7 \%$ & $12.3 \%$ & $59.6 \%$ & $25.3 \%$ \\
\hline $\begin{array}{l}\text { Businesses (i.e., outdoor or climbing related) } \\
N=148\end{array}$ & $3.4 \%$ & $8.1 \%$ & $59.5 \%$ & $29.1 \%$ \\
\hline $\begin{array}{l}\text { Climbing related organizations (Access } \\
\text { Fund, etc.) } \mathrm{N}=150\end{array}$ & $0 \%$ & $0 \%$ & $31.3 \%$ & $68.7 \%$ \\
\hline Scientists in general $\mathrm{N}=149$ & $4 \%$ & $18.8 \%$ & $55 \%$ & $22.1 \%$ \\
\hline National Park rangers in general $\mathrm{N}=147$ & $3.4 \%$ & $15.6 \%$ & $64.6 \%$ & $16.3 \%$ \\
\hline $\begin{array}{l}\text { National Park rangers at the New River } \\
\text { Gorge National River } N=149\end{array}$ & $2.7 \%$ & $12.8 \%$ & $65.5 \%$ & $19.5 \%$ \\
\hline Mainstream news media $\mathrm{N}=146$ & $43.2 \%$ & $42.5 \%$ & $13.7 \%$ & $0.7 \%$ \\
\hline \multicolumn{5}{|c|}{$\begin{array}{l}\text { Of the sources listed above, which do you most strongly trust, and WHY? } \\
\text { List source: } \\
\text { Why trust?: }\end{array}$} \\
\hline \multicolumn{5}{|c|}{$\begin{array}{l}\text { Of the sources listed above, which do you most strongly distrust, and WHY? } \\
\text { List source: } \\
\text { Why distrust?: }\end{array}$} \\
\hline
\end{tabular}

36. Do you currently belong to any of the following organizations affiliated with rock climbing or the environment? (check all that apply) $\mathrm{N}=86$
$\square$ Access Fund $55.8 \%$
$\square$ New River Alliance of Climbers 29.1\%
$\square$ Alpine Club of Canada 1.2\%
$\square$ American Alpine Club 16.3\%
$\square$ Leave No Trace 14\%
$\square$ American Mountain Guides Association 16.3\%
Nature Conservancy 3.5\%
$\square$ American Sport Climbing Federation 0\%
$\square$ Appalachian Mountain Club 17.4\%
$\square$ Other $22.1 \%$ (please write in

37. Did you know that volunteer climbers undertake maintenance projects at NRG? one) $\mathrm{N}=149$
$\square$ Yes $69.1 \%$
$\square$ No $30.9 \%$

38. Have you ever volunteered for a climbing area maintenance project at NRG (trail construction, trash clean up, etc.)? (check one) $\mathrm{N}=149$
$\square$ Yes $25.5 \% \square$ No $74.5 \%$

39. Have you ever volunteered for climbing area maintenance project anywhere else (trail construction, trash clean up, etc.)? (check one) $\mathrm{N}=150$

$\square$ Yes $52.7 \% \square$ No $47.3 \%$ 
40. Do you think rock climbers should share responsibility for climbing area maintenance? (check one) $\mathrm{N}=150$

$\square$ Yes $98.7 \% \square$ No $1.3 \%$

Finally, we would like to know a bit more about you.

41. What is your gender? (check one) $\mathrm{N}=150$

$\square$ Male $68 \% \square$ Female $32 \%$

42. What is the highest grade of school or year of college that you completed? (check one)

$\mathrm{N}=150$

$\square$ Less than high school $1.3 \% \quad \square$ Four year college degree $43.3 \%$

$\square$ High school graduate or GED 6.7\% $\square$ Masters, doctoral, or professional degree 22\%

$\square$ Some college or associate degree $26.7 \%$

43. Are you Hispanic or Latino? (check one) $\mathrm{N}=149$

$\square$ Yes $3.4 \% \quad \square$ No $96.6 \%$

44. Which racial groups do you identify with? (check one or more) N=135

$\square$ Native American or Alaska Native 3.5\%

$\square$ Asian $7 \%$

$\square$ Black or African American 0\%

$\square$ Native Hawaiian or other Pacific Islander $0.7 \%$

$\square$ White $94.4 \%$

45. What is your age? (please write in number)

46. Are you a permanent resident or citizen of the U.S.? (check one and fill in) $\mathrm{N}=150$

$\square$ No - What is your country of origin? $5.3 \%$

$\square$ Yes - What is your zip code, city and state of residence? 94.7\%

Zip Code

City

State

47. How many people (including yourself) are in your party on this visit?

(write in number)

44a. Which of the following best describes the group you are traveling with? (check one) $\mathrm{N}=149$ 
$\square$ Myself (alone) $8.7 \%$

Myself with family (including spouse/partner and/or other family members/relatives) $16.1 \%$

Myself with family and friends $10.1 \%$

Myself with friends $59.1 \%$

$\square$ Outfitter guided $4.7 \% \quad$ Specify

$\square$ Club/organization/school $0.7 \% \quad$ Specify

Other (Please specify) 07.\%

48. Do you consider yourself to be a leader or organizer for your group? $\mathrm{N}=145$

$$
\text { Yes } 51.7 \% \quad \square \text { No } 48.3 \%
$$

49. Are there any children under age 18 in your group? (check one \& write in number as needed) $\mathrm{N}=149$

$\square$ No $89.9 \%$

$\square$ Yes $\rightarrow$ How many children are in each of these age categories? 10.1\%

Number of children under 5 years old

Number of children age 5-12

Number of children age 13-17

\section{Thank you for your help!}

Privacy Act and Paperwork Reduction Act statement:

16 U.S.C. 1a-7 authorizes the National Park Service to collect this information to be used by park managers to better serve the public. Response to this request is voluntary. No action may be taken against you for refusing to supply the information requested. The Paperwork Reduction Act states that an agency may not conduct or sponsor, and a person is not required to respond to, a collection of information unless it displays a currently valid OMB control number and expiration date.

Burden estimate statement: Public reporting for this form is estimated to average 20 minutes per response. Direct comments regarding the burden estimate or any other aspect of this form to the Information Collection Clearance Officer, National Park Service, 1849 C Street, Washington, D.C. 20240. 